\title{
Global continuation of monotone waves for bistable delayed equations with unimodal nonlinearities
}

\author{
Sergei Trofimchuk \\ Instituto de Matemática y Fisica, Universidad de Talca, Casilla 747, Talca, Chile \\ E-mail: trofimch@inst-mat.utalca.cl

\section{Vitaly Volpert} \\ Institut Camille Jordan, UMR 5208 CNRS, University Lyon 1, 69622 Villeurbanne, \\ France \\ INRIA Team Dracula, INRIA Lyon La Doua, 69603 Villeurbanne, France \\ RUDN University, ul. Miklukho-Maklaya 6, Moscow, 117198, Russia \\ E-mail: volpert@math.univ-lyon1.fr
}

\begin{abstract}
We study the existence of monotone wavefronts for a general family of bistable reaction-diffusion equations with delayed reaction term $g$. Differently from previous works, we do not assume the monotonicity of $g(u, v)$ with respect to the delayed variable $v$ that does not allow to apply the comparison techniques. Thus our proof is based on a variant of the Hale-Lin functional-analytic approach to heteroclinic solutions of functional differential equations where Lyapunov-Schmidt reduction is done in appropriate weighted spaces of $C^{2}$-smooth functions. This method requires a detailed analysis of associated linear differential Fredholm operators and their formal adjoints. For two different types of $v$-unimodal functions $g(u, v)$, we prove the existence of a maximal continuous family of bistable monotone wavefronts.. Depending on the type of unimodality (equivalently, on the sign of the wave speed), two different scenarios can be observed for the bistable waves: 1) independently on the size of delay, each bistable wavefront is monotone; 2) wavefronts are monotone for moderate values of delays and can oscillate for large delays.
\end{abstract}

AMS classification scheme numbers: 34K12, 35K57, 92D25

Keywords: bistable equation, monotone wavefront, non-monotone reaction, existence 


\section{Introduction and main results}

The main objects of investigation in this work are traveling front solutions for the delayed reaction-diffusion equation

$$
u_{t}(t, x)=u_{x x}(t, x)+g(u(t, x), u(t-\tau, x)), \quad u \in \mathbb{R},(t, x) \in \mathbb{R}^{2}, \tau \geq 0,
$$

in the particular case when the reaction term $g$ satisfies the following bistability condition:

(B) Function $g$ is $C^{1, \gamma}$-continuous on some set $(\alpha, \beta)^{2} \subset \mathbb{R}^{2}$. On the interval $(\alpha, \beta)$, equation $g(u, u)=0$ has exactly three solutions $e_{1}<e_{2}<e_{3}$ such that $g_{1}\left(e_{j}, e_{j}\right)+g_{2}\left(e_{j}, e_{j}\right)<0$ and $g_{1}\left(e_{j}, e_{j}\right)<0$ for $j=1,3$ (in the paper, we use the notations $g_{j}\left(u_{1}, u_{2}\right), j=1,2$, for partial derivatives $\left.\partial g\left(u_{1}, u_{2}\right) / \partial u_{j}, j=1,2\right)$.

We recall that classical solution $u(t, x)=\phi(x+c t)$ of $(1)$ is called a bistable traveling front (in the sequel, we shorten this name to the word 'wavefront' which will be used both for the solution $u(t, x)=\phi(x+c t)$ and for its profile $\phi(s))$ propagating with the velocity $c$, if $\phi$ is bounded $C^{2}$-smooth function satisfying $\phi(-\infty)=e_{1}$ and $\phi(+\infty)=e_{3}$. Wavefront is called monotone if $\phi^{\prime}(t) \geq 0, t \in \mathbb{R}$. Replacing in the above definition the boundary condition $\phi(+\infty)=e_{3}$ with a weaker restriction lim $\inf _{t \rightarrow+\infty}>e_{2}$, we define a classical solution called a semi-wavefront. It is clear that each wavefront $\phi$ to (1) has to satisfy the following boundary value problem for delayed differential equation

$$
\phi^{\prime \prime}(t)-c \phi^{\prime}(t)+g(\phi(t), \phi(t-c \tau))=0, \quad t \in \mathbb{R}, \quad \phi(-\infty)=e_{1}, \phi(+\infty)=e_{3} .
$$

There are several particular forms of problem (2) for which the existence of solutions is known. The simplest of them appears when $c \tau=0$ : problem (2) is then without delay and it is well understood [31]. In consequence, we are interested only in nonstationary wavefronts and will consider speed $c \neq 0$. Another well studied particular case of $(2)$ is when the nonlinearity $g(u, v)$ is non-decreasing in $v$ for each fixed $u$ $[7,12,14,22,24,27,28,32]$. Indeed, this kind of monotonicity allows a successful application of the maximum principle and comparison techniques.

However, if the condition $g_{2}(u, v) \geq 0$ does not hold, not so much is known even about the existence of wavefronts to delayed reaction-diffusion equation (1). $\dagger$ In fact, we are aware about only two such works, [1, 29], where special cases of equation (1) were analysed by means of the Leray-Schauder topological degree argument. In particular, the following model

$$
u_{t}(t, x)=u_{x x}(t, x)-u(t, x)+f(u(t-\tau, x)), \quad u \geq 0, \quad(t, x) \in \mathbb{R}^{2},
$$

with $C^{1, \gamma}$-continuous nonlinearity $f:[0,+\infty) \rightarrow[0,+\infty)$ satisfying

$$
f(0)=0=: e_{1}, f^{\prime}(0) \in(0,1], f\left(e_{2}\right)-e_{2}=f\left(e_{3}\right)-e_{3}=0
$$

has been recently considered in [1]. Note that bistable equation (3) with the unimodal birth function $f$ having only three fixed points, $e_{1}, e_{2}, e_{3}$, is broadly used in the $\dagger$ And practically nothing is known about the uniqueness of bistable wavefronts in the non-monotone case, cf. [1]. 
mathematical ecology for modelling systems exhibiting the Allee effect, cf. [4, Fig. 1d]. Here, the unimodality of $f$ means that $f$ is hump-shaped, i.e. it has a unique critical point, $\kappa$, and $0<e_{2}<\kappa<e_{3}$. In [14], the above equation with unimodal $f$ was classified as Type D nonlinearity (see [14, Fig. 4.4]) and it was noted in [14, p. 5133] that there has been no progress for Type D at the moment of the publication of [14]. In this regard, the recent contribution [1] by Alfaro, Ducrot and Giletti presents a series of important existence results. Under some general bistability type assumptions on $f$ (which imply the positivity of the speed of propagation but are weaker than the unimodality restriction), Alfaro et al proved the existence of semi-wavefront solutions to (3) and established conditions sufficient for their either convergence or oscillation at $+\infty$. In this paper, by giving a criterion for the existence of monotone wavefronts to (1), we provides an additional insight into the interesting findings of [1].

Another type of bistable equation (1) with unimodal nonlinearity was recently proposed in [6] in order to understand spatiotemporal dynamics of virus infection spreading in tissues. The model equation of [6] is of the form

$$
u_{t}(t, x)=u_{x x}(t, x)+u(t, x)(1-u(t, x)-f(u(t-\tau, x))), \quad u \geq 0,(t, x) \in \mathbb{R}^{2} .
$$

It is assumed that $f: \mathbb{R}_{+} \rightarrow(0,+\infty)$ is $C^{1, \gamma}$-continuous function and that the equation $1-u=f(u)$ has exactly three positive solutions $0<e_{1}<e_{2}<e_{3}$ on the interval $[0,1]$. In addition, $f^{\prime}\left(e_{1}\right) \geq 0, f^{\prime}\left(e_{3}\right)>-1$ and $f$ achieves the global maximum at its unique critical point $\kappa \in\left(e_{1}, e_{2}\right)$. See Fig. 1 in [6]. The recent work [29] establishes that a simpler version of (4) (with $e_{1}<0<e_{2}<e_{3}=1$ ) has at least one monotone wavefront connecting the equilibria 0 and 1 for each fixed delay $\tau \geq 0$.

The above mentioned biological models show the importance of studying the existence of wavefronts for equation (1) with the reaction term $g(u, v)$ which is not increasing in the second variable, but still has reasonably good (piece-wise monotone, with only two pieces of monotonicity) behavior with respect to $v$ for each fixed $u$. We will include both models (3) and (4) in our general theory by considering two following alternative unimodality assumptions:

(U) For each fixed $u \in(\alpha, \beta)$, function $g(u, \cdot)$ has a unique critical point $\kappa \in\left(e_{1}, e_{2}\right)$, independent on $u$ (hence, $g_{2}(u, \kappa)=0$ ) such that $g_{2}(u, v)<0$ for $v \in(\alpha, \kappa)$ and $g_{2}(u, v)>0$ for $v \in(\kappa, \beta)$. Furthermore, $g_{1}(u, v)<0$ for all $u \geq v$ such that $u \in\left[e_{1}, e_{2}\right)$, $v \in\left[e_{1}, \kappa\right]$ and $g\left(u, e_{1}\right)<0$ for all $u \in\left(e_{2}, \beta\right)$ while $g\left(u, e_{1}\right)>0$ for all $u \in\left(\alpha, e_{1}\right)$. (The latter implies that $g(u, v)<0$ for all $\left.u \geq v, u, v \in\left(e_{1}, e_{2}\right)\right)$.

( $\left.\mathbf{U}^{*}\right)$ For each fixed $u \in(\alpha, \beta)$, function $g(u, \cdot)$ has a unique critical point $\kappa \in\left(e_{2}, e_{3}\right)$, independent on $u$ such that $g_{2}(u, v)>0$ for $v \in(\alpha, \kappa)$ and $g_{2}(u, v)<0$ for $v \in(\kappa, \beta)$. Furthermore, $g_{1}(u, v)<0$ for all $u \geq v ; u, v \in\left[\kappa, e_{3}\right]$. In addition, $g(u, v)$ is 'strongly' sub-tangential at $e_{3}: g_{j}(u, v) \geq g_{j}\left(e_{3}, e_{3}\right), j=1,2, u \geq v, u, v \in\left[e_{1}, e_{3}\right]$.

There is certain asymmetry in the strength of assumptions $(\mathbf{U})$ and $\left(\mathbf{U}^{*}\right)$ : in particular, the sub-tangency requirement of $\left(\mathbf{U}^{*}\right)$ is used repeatedly in the proof of one of our main results, Theorem 1.4. Clearly, the 'strong' sub-tangency condition is 
somewhat stronger than the usual sub-tangency requirement $g(u, v) \leq g_{1}\left(e_{3}, e_{3}\right)(u-$ $\left.e_{3}\right)+g_{2}\left(e_{3}, e_{3}\right)\left(v-e_{3}\right), u \geq v, u, v \in\left[e_{1}, e_{3}\right]$. On the other hand, the form of subtangency given in $\left(\mathbf{U}^{*}\right)$ seems to be more friendly for applications. For instance, it is easy to see that the reaction term in (3) satisfies $\left(\mathbf{U}^{*}\right)$ if $f^{\prime}\left(e_{3}\right)=\min \left\{f^{\prime}(u), u \in\left[e_{1}, e_{3}\right]\right\}$ (note also that hypothesis (U) holds for equation (4) without additional restrictions on f). Importantly, in Section 2 we show how a slightly weaker version of Theorem 1.4, Theorem 2.5, can be obtained without any kind of sub-tangency restriction at $e_{3}$.

As we have mentioned, in this paper we consider only non-stationary wavefronts. In fact, it suffices to analyse the case of positive speeds, $c>0$, since the linear change of variables $\psi(-t)=e_{1}+e_{3}-\phi(t)$ transforms problem (2) under assumption (U) and with the speed $c$ into problem (2) under assumption $\left(\mathbf{U}^{*}\right)$ and with the speed $-c$, and vice versa (of course, modulo the sub-tangency condition and secondary monotonicity details). In the next section, we are applying this trick in the case of models (3) and (4). Note also that if velocity $c$ is positive then traveling front is an expansion wave (since $\phi(x+c t)$ converges, uniformly on compact sets, to the biggest steady state $e_{3}$ as $t \rightarrow+\infty$ ). As we show, for the positivity of speed (for each fixed $\tau \geq 0$ ) it is enough to assume the inequality

(I) $\mathfrak{I}:=\int_{e_{1}}^{e_{3}} g(u, u) d u>0$.

Now, even if equation (1) generally defines a non-monotone evolutionary system, we are interested in the existence of monotone wavefronts for it, cf. [5, 11, 12, 13, 18, 29]. In the paper, such wavefronts will be obtained via deformation of the unique monotone wavefront of equation (1) considered with $\tau=0$. The procedure of this continuous deformation requires from solutions of (1) the following monotonicity property (satisfied for both considered biological models), cf. [13, 29, 31]:

(M) Suppose that $u=\phi(x+c t), c>0$, is a non-decreasing wavefront connecting the steady states $e_{1}$ and $e_{3}$. Then $\phi^{\prime}(t)>0, t \in \mathbb{R}$.

Monotonicity of the initial wavefront should be preserved during its continuous deformation. It appears that it is easier to satisfy this requirement under assumption (U) than under ( $\left.\mathbf{U}^{*}\right)$. Indeed, as we will show in Lemmas 3.3, 3.4, 3.5, 3.7, (U) assures that each wavefront is strictly increasing at $\pm \infty$ and it is confined between the equilibria $e_{1}$ and $e_{3}$. Contrary to this, if $\left(\mathbf{U}^{*}\right)$ is assumed then it is easy to control monotonicity at $-\infty$ but not at $+\infty$ (asymptotic behaviour of monotone wavefronts at $+\infty$ is described in terms of zeros of the associated characteristic function $\chi_{-}(z)=z^{2}-c z+a_{-}+b_{-} e^{-z c \tau}$, where coefficients $a_{-}, b_{-}$are negative, see Section 4$)$. A similar difficulty has occurred in [13] during the continuous deformation of monostable monotone wavefronts. In the cited work, it has been shown that the monotone deformation of wavefronts can still be realised inside of some domain $\mathcal{D}$ of parameters $(\tau, c)$ described in continuation. To define $\mathcal{D}$, we need the following result from [13, Lemma 1.1] concerning the real zeros of $\chi_{-}(z)$ : 
Proposition 1.1 Given $a_{-}+b_{-}<0, b_{-}<0$, there exists $\operatorname{clin}(\tau) \in(0,+\infty]$ such that the characteristic equation $\chi_{-}(z)=0, c>0$, has three real roots $\lambda_{1} \leq \lambda_{2}<0<\lambda_{3}$ if and only if $c \leq \operatorname{clin}(\tau)$. If $\operatorname{clin}(\tau)$ is finite and $c=\operatorname{clin}(\tau)$, then $\chi_{-}(z)$ has a double zero $\lambda_{1}=\lambda_{2}<0$, while for $c>$ clin $(\tau)$ there does not exist any negative root to $\chi_{-}(z)=0$. Moreover, if $\lambda_{j} \in \mathbb{C}$ is a complex root of $\chi_{-}(z)=0$ for $c \in(0, \operatorname{clin}(\tau)]$ then $\Re \lambda_{j}<\lambda_{2}$.

Furthermore, $\operatorname{clin}(\tau)=+\infty$ for all $\tau$ from some non-empty maximal interval $\left[0, \tau_{\#}\right]$ and $\operatorname{clin}(\tau)$ is strictly decreasing on $\left(\tau_{\#},+\infty\right)$. In fact,

$$
\operatorname{clin}(\tau)=\frac{\theta\left(a_{-}, b_{-}\right)+o(1)}{\tau}, \quad \tau \rightarrow+\infty, \quad \text { where } \theta\left(a_{-}, b_{-}\right):=\sqrt{\frac{2 \omega}{b_{-}}} e^{\omega / 2},
$$

and $\omega$ is the unique negative root of $-2 a_{-}=b_{-} e^{-\omega}(2+\omega)$.

Remark 1.2 Suppose that $a_{-}<0$, then a straightforward analysis shows that $\tau_{\#}>0$ can be determined as the unique real root of the equation e $\left|b_{-}\right| \tau e^{\left|a_{-}\right| \tau}=1$. For $\tau>\tau_{\#}$, the function $c=\operatorname{clin}(\tau)$ can be defined implicitly by $f$ the equation

$\mathfrak{A}(c, h):=\frac{2+\sqrt{c^{2} h^{2}+4+4\left|a_{-}\right| h^{2}}}{e h^{2}\left|b_{-}\right|}=\exp \left(\frac{2+2\left|a_{-}\right| h^{2}}{c h+\sqrt{c^{2} h^{2}+4+4\left|a_{-}\right| h^{2}}}\right)=: \mathfrak{B}(c, h),(5)$ where $h=c \tau$. Figures 2, 3 below present the graph of $c=\operatorname{clin}(\tau)$ for $\left|a_{-}\right|=\left|b_{-}\right|=1$.

We define $\mathfrak{D}\left(a_{-}, b_{-}\right)$as the set of non-negative parameters for which $\chi_{-}(z), c>0$, has exactly three real zeros (counting multiplicity). In the coordinates $(\tau, c)$, this domain takes the next form

$$
\mathfrak{D}\left(a_{-}, b_{-}\right)=\{(\tau, c): \tau \geq 0,0<c \leq \operatorname{clin}(\tau)\} \subset \mathbb{R}_{+}^{2} .
$$

We can now state the first main result of the paper:

Theorem 1.3 Let assumptions (B), (I), (M) and (U) be satisfied. Then equation (1) has a continuous family of strictly increasing bistable wavefronts $u=\phi(x+c(\tau) t, \tau)$, $\tau \geq 0$, propagating with the positive speed $c=c(\tau)$.

In Subsection 2.3, we show (see Figure 3 below) that, under assumptions of Theorem 1.3, it might happen that $c(\tau)>\operatorname{clin}(\tau)$ for some positive values of $\tau$. Quite the contrary, under assumption $\left(\mathbf{U}^{*}\right)$, we need the condition

$(\tau, c(\tau)) \in \operatorname{Int} \mathfrak{D}\left(g_{1}\left(e_{3}, e_{3}\right), g_{2}\left(e_{3}, e_{3}\right)\right)$, where Int $\mathfrak{D}$ denotes the interior of the domain $\mathfrak{D}$, in order to realise monotone deformation of the initial wavefront:

Theorem 1.4 Let assumptions (B), (I), (M) and ( $\left.\mathbf{U}^{*}\right)$ be satisfied. Then there exists an extended real number $\tau_{*}>\tau_{\#}$ and a continuous function $c=c(\tau), \tau \in\left[0, \tau_{*}\right]$, such that equation (1) has a continuous family of strictly increasing bistable wavefronts $u=\phi(x+c(\tau) t, \tau), \tau \leq \tau_{*}$, propagating with the positive speed $c=c(\tau)$. Moreover, $(\tau, c(\tau)) \in \mathfrak{D}\left(g_{1}\left(e_{3}, e_{3}\right), g_{2}\left(e_{3}, e_{3}\right)\right), c\left(\tau_{*}\right)=\operatorname{clin}\left(\tau_{*}\right)$, and $\left[0, \tau_{*}\right]$ is the maximal interval (containing 0) for the existence of monotone wavefronts. Furthermore, if $\tau_{*}$ is finite, then there is a sequence of delays $\tau_{j} \rightarrow \tau_{*}$ such that equation (1) considered with $\tau=\tau_{j}$ has a wavefront propagating with speed $c_{j}, c_{j} \rightarrow$ clin $\left(\tau_{*}\right)$, and oscillating around $e_{3}$. 
In the next section, we apply Theorems 1.3 and 1.4 to models (3) and (4). In particular, we prove that condition (M) is fulfilled for these equations. Not only positive but also negative speeds of propagation are considered. In addition, in Subsection 2.2, we state a somewhat weaker version of Theorem 1.4, Theorem 2.5. This result does not require any sub-tangency restriction from $g$. In Subsection 2.3, we are also illustrating our findings on an explicit example allowing a rather complete analytical and numerical analysis (this type of 'toy models' was proposed in [26], see also [10, 17]). In particular, the computations done in Subsection 2.3 suggest that $c=c(\tau)$ is decreasing function of $\tau$ and that each monotone wavefront is unique (up to a translation).

As in [13], our proofs are based on the homotopy method and a variant of Hale-Lin functional-analytic approach to the heteroclinic solutions [15]. In the bistable setting, this theory was developed further by S.-N. Chow, X.-B. Lin, J. Mallet-Paret and W. Huang in $[8,18,19,25]$. In this theory, application of the Lyapunov-Schmidt reduction requires a thorough analysis of the variational equations (and their adjoints) along the monotone wavefronts. Variational equations are analysed in Section 3 (under assumption $(\mathbf{U})$ ) and Section 4 (under assumption $\left(\mathbf{U}^{*}\right)$ ). The main conclusion of these sections concerns the existence of positive (either on $\mathbb{R}$ or $\mathbb{R}_{+}$) solutions $w_{*}(t)$ of the adjoint equations (Lemmas 3.13 and 4.8). Finally, Theorems 1.3 and 1.4 are proved in Section 5: to deal with the case when $w_{*}(t)$ can take negative values at some points $t<0$, we make appropriate adjustments (expressed in terms of corrector functions) to the Lyapunov-Schmidt procedure.

\section{Two biological models and one illustrative example.}

In this section, we consider three different nonlinearities $g$ and, in each case, we apply the main results of the paper, Theorems 1.3 and Theorems 1.4, to establish the existence of monotone (oscillating) wavefronts propagating with positive and negative speeds.

\subsection{Mackey-Glass type model (3).}

Assume that $C^{1, \gamma}$-continuous unimodal function $f: \mathbb{R}_{+} \rightarrow \mathbb{R}_{+}$satisfies

a1) $f(0)=0=: e_{1}, f^{\prime}(0) \in(0,1), f\left(e_{2}\right)-e_{2}=f\left(e_{3}\right)-e_{3}=0$. We also assume that equation $f(x)=x$ has only three solutions, $e_{1}, e_{2}, e_{3}$;

a2) $f^{\prime}\left(e_{3}\right) \leq f^{\prime}(x), x \in\left[0, e_{3}\right]$;

a3) the unique critical point $\kappa$ of $f$ belongs to the interval $\left(e_{2}, e_{3}\right)$.

Then $g(u, v)=-u+f(v)$ meets all restrictions of $(\mathbf{B}),\left(\mathbf{U}^{*}\right)$. The wave profile equation for $(3)$ is

$\phi^{\prime \prime}(t)-c \phi^{\prime}(t)-\phi(t)+f(\phi(t-c \tau))=0, \quad t \in \mathbb{R}, \quad \phi(-\infty)=e_{1}, \phi(+\infty)=e_{3}$.

We claim that condition (M) is satisfied in such a case. Indeed, let $\phi(t)$ be a profile of a bistable wave such that $\phi^{\prime}(t) \geq 0, \phi(-\infty)=e_{1}, \phi(+\infty)=e_{3}$. Then Lemma 4.4 says that there exists a maximal interval $(-\infty, r)$ such that $\phi^{\prime}(t)>0$ 
for all $t<r$. In addition, it holds that $\phi(r) \geq e_{2}$. Suppose that $r$ is finite, then $\phi^{\prime}(r)=\phi^{\prime \prime}(r)=0$ so that $\phi(r)=f(\phi(r-c \tau))$. After differentiating (6), we also obtain that $\phi^{\prime \prime \prime}(r)=-f^{\prime}(\phi(t-c \tau)) \phi^{\prime}(r-c \tau)$. Since $\phi^{\prime \prime \prime}(r) \geq 0$ and $\phi^{\prime}(r-c \tau)>0$, we find that $f^{\prime}(\phi(r-c \tau)) \leq 0$. Thus $\phi(r-c \tau) \geq \kappa$ so that $\phi(r) \leq e_{3}<f(\phi(r-c \tau))$, a contradiction. Hence, Theorem 1.4 applies in such a case:

Theorem 2.1 Let assumptions a1),a2), a3) be satisfied together with (I) which here reads as

$$
\mathfrak{P}:=\frac{1}{e_{3}-e_{1}} \int_{e_{1}}^{e_{3}} f(u) d u-\frac{e_{1}+e_{3}}{2}>0 .
$$

Then all conclusions of Theorem 1.4 are valid for equation (3).

In Subsection 2.3, we present an explicit example showing that the Mackey-Glass type bistable models can have wavefronts oscillating around $e_{3}$.

Next, in order to investigate the existence of monotone wavefronts for equation (6) when $\mathfrak{P}<0$ we may apply, as it was suggested in the introduction, the change of variables $\psi(-t)=e_{1}+e_{3}-\phi(t)$. It transforms the original equation into equation (2) with new reaction term $\tilde{g}(u, v)=e_{1}+e_{3}-u-f\left(e_{1}+e_{3}-v\right)$, steady states $e_{1}<\tilde{e}_{2}=e_{1}+e_{3}-e_{2}<e_{3}$ and the critical point $\tilde{\kappa}=e_{1}+e_{3}-\kappa \in\left(e_{1}, \tilde{e}_{2}\right)$. Moreover, it can be checked easily that $\tilde{g}(u, v)$ satisfies (B), (U), (I) if we assume conditions a1), a3) and $\mathfrak{P}<0$.

Finally, let $\psi(t)$ be a wavefront for the modified equation satisfying $\psi^{\prime}(t) \geq 0$, $\psi(-\infty)=e_{1}, \psi(+\infty)=e_{3}$. Then Lemma 3.3 says that there exists a maximal interval $(-\infty, r)$ such that $\psi^{\prime}(t)>0$ for all $t<r$. In addition, $\psi(r-|c| \tau)>\tilde{\kappa}$. Suppose that $r$ is finite, then $\psi^{\prime}(r)=\psi^{\prime \prime}(r)=0$ so that $e_{1}+e_{3}-\psi(r)=f\left(e_{1}+e_{3}-\psi(r-|c| \tau)\right)$, $\psi^{\prime \prime \prime}(r)=-f^{\prime}\left(e_{1}+e_{3}-\psi(r-|c| \tau)\right) \psi^{\prime}(r-|c| \tau)$. Since $e_{1}+e_{3}-\psi(r-|c| \tau)<\kappa$, we conclude that $\psi^{\prime \prime \prime}(r)<0$, a contradiction. Hence, condition (M) is satisfied by $\psi(t+|c| t)$ and an application of Theorem 1.3 leads to the following result.

Theorem 2.2 Assume conditions a1), a3) as well as the inequality $\mathfrak{P}<0$. Then for each $\tau \geq 0$ equation (6) has a monotone wavefront propagating with the negative speed $c(\tau)$ which depends continuously on the delay $\tau$.

\subsection{A model of virus infection spreading in tissues.}

Following [6], we consider reaction-diffusion equation (4) with the unimodal $C^{1, \gamma_{-}}$ continuous function $f: \mathbb{R}_{+} \rightarrow(0,+\infty)$ such that

b1) equation $1-u=f(u)$ has exactly three positive solutions $0<e_{1}<e_{2}<e_{3}<1$ on the interval $[0,1]$ and $f^{\prime}\left(e_{1}\right) \geq 0, f^{\prime}\left(e_{3}\right)>-1$;

b2) $f$ has a unique critical point $\kappa \in\left(e_{1}, e_{2}\right)$ where the global maximum of $f$ is achieved. Then the function $g(u, v)=u(1-u-f(v))$ clearly satisfies the assumptions (B) (where $(\alpha, \beta)=(0,1))$ and $(\mathbf{U})$. Next, each bistable wavefront $\phi$ for model (4) solves the boundary problem

$\phi^{\prime \prime}(t)-c \phi^{\prime}(t)+\phi(t)(1-\phi(t)-f(\phi(t-c \tau)))=0, \phi(-\infty)=e_{1} \geq 0, \phi(+\infty)=e_{3}$. 
The assumption (M) is also satisfied because of the following proposition.

Lemma 2.3 Suppose that $\phi(t)$ satisfies (7). If $\phi(t)$ is non-decreasing on some interval $(-\infty, s]$ and $\phi(t) \in\left[e_{2}, e_{3}\right) \quad$ for $t \geq s$, then $\phi^{\prime}(t)>0, t \in \mathbb{R}$.

Proof. Let $s^{\prime}<s$ be a critical point for $\phi(t)$. Then $\phi^{\prime}\left(s^{\prime}\right)=\phi^{\prime \prime}\left(s^{\prime}\right)=0$ so that $f\left(\phi\left(s^{\prime}-c \tau\right)\right)=1-\phi\left(s^{\prime}\right)$. Since $\phi\left(s^{\prime}\right) \geq \phi\left(s^{\prime}-c \tau\right)$ the latter equality implies that $\phi\left(s^{\prime}-c \tau\right), \phi\left(s^{\prime}\right)>e_{2}$. Hence, $\phi(s-c \tau), \phi(s)>e_{2}$. Clearly, we may assume that $s=\sup \left\{r: \phi^{\prime}(t) \geq 0, t \in(-\infty, r]\right\}$ and that there is $s_{*} \leq s$ such that $\phi^{\prime}(t)>0$ for $t<s_{*}, \phi^{\prime}\left(s_{*}\right)=0$. We have that either $s_{*}=s$ or $s_{*}<s$ and $\phi^{\prime \prime}\left(s_{*}\right)=0$. In the latter case, $\phi\left(s_{*}-c \tau\right)>e_{2}$ and

$$
\phi^{\prime \prime \prime}\left(s_{*}\right)=\phi\left(s_{*}\right) f^{\prime}\left(\phi\left(s_{*}-c \tau\right)\right) \phi^{\prime}\left(s_{*}-c \tau\right)<0,
$$

a contradiction. Thus $\phi^{\prime}(t)>0$ for all $t<s$ and, in addition, if $s$ is finite then $\phi^{\prime \prime}(s)<0$. Hence, if $s$ is finite, then $\phi^{\prime}(t)<0$ on some maximal interval $(s, S)$ (where $S$ is finite because of the condition $\left.\phi(+\infty)=e_{3}\right)$. Evidently, $\phi^{\prime}(S)=0, \phi^{\prime \prime}(S) \geq 0$ so that $1-\phi(S) \leq f(\phi(S-c \tau))$. First, suppose that $S-c \tau \geq s$. Then $\phi(s) \geq \phi(S-c \tau)>$ $\phi(S) \geq e_{2}$ implying that

$$
1-\phi(S) \leq f(\phi(S-c \tau))<f(\phi(S))
$$

a contradiction (since $f(x) \leq 1-x$ on $\left[e_{2}, e_{3}\right]$ ). In consequence, $S-c \tau<s$ so that

$$
e_{2}<\phi(s-c \tau)<\phi(S-c \tau)<\phi(s)>\phi(S)
$$

yielding again a contradiction:

$$
1-\phi(s)>f(\phi(s-c \tau))>f(\phi(S-c \tau)) \geq 1-\phi(S) .
$$

Thus $s=+\infty$ and $\phi^{\prime}(t)>0$ for all $t \in \mathbb{R}$.

An application of Theorem 1.3 allows us to extend the main existence result of [29] on model (7) considered under more realistic settings, cf. [6] :

Theorem 2.4 Assume conditions b1),b2) as well as condition (I) which here is equivalent to

$$
\mathfrak{J}:=\int_{e_{1}}^{e_{3}} u(1-u-f(u)) d u>0 .
$$

Then for each $\tau \geq 0$ equation (7) has a monotone wavefront propagating with the positive speed $c(\tau)$ which depends continuously on the delay $\tau$.

Finally, supposing that $\mathfrak{J}<0$, we will study the existence of wavefronts propagating with negative speeds (i.e. of the extinction waves). Since we are going to invoke Theorem 1.4, this suggests the use of the transform $\psi(-t)=e_{1}+e_{3}-\phi(t)$. The new reaction term has the form $\hat{g}(u, v)=-\left(e_{1}+e_{3}-u\right)\left(1-e_{1}-e_{3}+u-f\left(e_{1}+e_{3}-v\right)\right)$ and it is immediate to see that the 'strong' sub-tangency condition of $\left(\mathbf{U}^{*}\right)$ is not satisfied by $\hat{g}_{1}(u, v)=1-2\left(e_{1}+e_{3}-u\right)-f\left(e_{1}+e_{3}-v\right), \quad g_{2}(u, v)=-\left(e_{1}+e_{3}-u\right) f^{\prime}\left(e_{1}+e_{3}-v\right)$.

In this case, it is convenient to apply the following weaker version of Theorem 1.4: 
Theorem 2.5 Let assumptions (B), (I), (M) and ( $\left.\mathbf{U}^{*}\right)$ (except for the 'strong' subtangency condition) be satisfied. Set

$\tilde{a}_{-}=\min \left\{g_{1}(u, v): u \geq v, u, v \in\left[e_{1}, e_{3}\right]\right\}, \tilde{b}_{-}=\min \left\{g_{2}(u, v): u \geq v, u, v \in\left[e_{1}, e_{3}\right]\right\}(8)$ and let $\tilde{\tau}_{\#}>0$ be the unique real root of the equation $e\left|\tilde{b}_{-}\right| \tau e^{\left|\tilde{a}_{-}\right| \tau}=1$. Then there exists an extended real number $\tau_{*}>\tilde{\tau}_{\#}$ and a continuous function $c=c(\tau), \tau \in\left[0, \tau_{*}\right]$, such that equation (1) has a continuous family of strictly increasing bistable wavefronts $u=\phi(x+c(\tau) t, \tau), \tau \leq \tau_{*}$, propagating with a positive speed $c=c(\tau)$. Moreover, $(\tau, c(\tau)) \in \mathfrak{D}\left(\tilde{a}_{-}, \tilde{b}_{-}\right)$and the point $\left(\tau_{*}, c\left(\tau_{*}\right)\right)$ belongs to the boundary of domain $\mathfrak{D}\left(\tilde{a}_{-}, \tilde{b}_{-}\right)$.

The strategy of The Proof of Theorem 2.5. The 'strong' sub-tangency condition of $\left(\mathbf{U}^{*}\right)$ is invoked four times in the proof of Theorem 1.4. In Remarks 4.2, 4.10, 5.10, 5.11 below, we show how the exclusion of this condition changes the proof and the conclusion of Theorem 1.4.

Computing the parameters $\tilde{a}_{-}, \tilde{b}_{-}$and then applying Theorem 2.5 to the transformed version of equation (7), we obtain the following.

Theorem 2.6 Assume conditions b1), b2) and the inequality $\mathfrak{J}<0$. Set

$$
\tilde{a}_{-}=\min \left\{1-2 u-f(u): u \in\left[e_{1}, e_{3}\right]\right\}, \quad \tilde{b}_{-}=\min \left\{-u f^{\prime}(u): u \in\left[e_{1}, e_{3}\right]\right\}
$$

and define $\tilde{\tau}_{\#}>0$ as in Theorem 2.5. Then there is an extended real number $\tau_{*}>\tilde{\tau}_{\#}$ and a continuous function $c=c(\tau), \tau \in\left[0, \tau_{*}\right]$ such that model (4) has a continuous family of strictly increasing bistable wavefronts $u=\phi(x+c(\tau) t, \tau), \tau \leq \tau_{*}$, propagating with negative speed $c=c(\tau)$. Moreover, $(\tau,|c(\tau)|) \in \mathfrak{D}\left(\tilde{a}_{-}, \tilde{b}_{-}\right)$and the point $\left(\tau_{*},\left|c\left(\tau_{*}\right)\right|\right)$ belongs to the boundary of the domain $\mathfrak{D}\left(\tilde{a}_{-}, \tilde{b}_{-}\right)$.

Proof. It is immediate to see that the function $\hat{g}(u, v)=-\left(e_{1}+e_{3}-u\right)\left(1-e_{1}-e_{3}+u-\right.$ $\left.f\left(e_{1}+e_{3}-v\right)\right)$ meets conditions (B), (I) and ( $\left.\mathbf{U}^{*}\right)$ (except for the 'strong' sub-tangency requirement). Now, the validity of assumption ( $\mathrm{M})$ for the transformed equation follows from a similar property of the original model (7) considered with $c<0$ : every its nondecreasing wavefront $\phi(t)$ connecting $e_{1}$ and $e_{3}$ satisfies the inequality $\phi^{\prime}(t)>0, t \in \mathbb{R}$. In order to demonstrate this property, on the contrary, suppose that the set $\mathcal{S} \subset \mathbb{R}$ of all critical points of $\phi$ is non-empty. Then $\phi^{\prime \prime}(s)=0$ for each $s \in \mathcal{S}$ and, consequently, $1-\phi(s)=f(\phi(s-c \tau))$. Since $c<0$, this implies that $\phi(s) \in\left(e_{1}, e_{2}\right), \phi(s-c \tau) \in\left(\kappa, e_{2}\right)$ so that $\sup \mathcal{S}=: s_{0} \in \mathcal{S}$ is finite. After differentiating (7) at $s_{0}$, we obtain the following contradiction $0 \leq \phi^{\prime \prime \prime}\left(s_{0}\right)=\phi\left(s_{0}\right) f^{\prime}\left(\phi\left(s_{0}-c \tau\right)\right) \phi^{\prime}\left(s_{0}-c \tau\right)<0$. Hence, $\mathcal{S}=\emptyset$ and Theorem 2.5 can be applied to the equation with transformed reaction term $\hat{g}(u, v)$.

\subsection{A 'toy' model.}

In this subsection, we are going to illustrate results concerning the Mackey-Glass type model by considering in (6) the following discontinuous nonlinearity

$$
f(u)=\left\{\begin{array}{cc}
p u, & u \in[0, \kappa), \\
1+q(u-1), & u \geq \kappa,
\end{array}\right.
$$


with $\kappa, p \in(0,1), q<0$ and $e_{1}=0, e_{3}=1$, see Figure 1 .
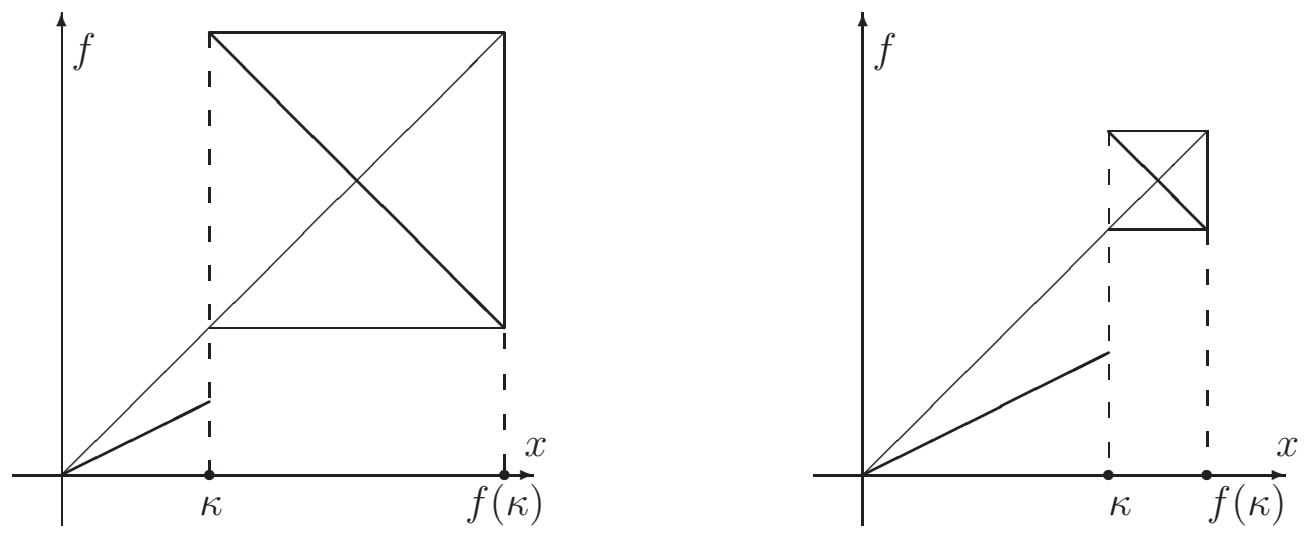

Figure 1. Graph of the unimodal birth function $f$ : the cases $c>0$ (on the left) and $c<0$ (on the right).

First, we assume the inequality $(\mathbf{I}): \int_{0}^{1}(-u+f(u)) d u>0$, see Figure 1 , left. This condition amounts to

$$
k_{*}:=\kappa\left(1+\sqrt{\frac{1-p}{1-q}}\right)<1
$$

Let $\phi$ be a profile of a bistable wave normalised by the condition $\phi(-c \tau)=\kappa$. Clearly, $\phi$ is a positive solution of the linear equation

$$
\phi^{\prime \prime}(t)-c \phi^{\prime}(t)-\phi+p \phi(t-c \tau)=0, \quad t<0 .
$$

The characteristic equation for (10) is

$$
\lambda^{2}-c \lambda-1+p e^{-c \tau \lambda}=0,
$$

and it has a unique positive real root $\mu_{1}=\mu_{1}(c, \tau)$, see also Lemma 3.1 below. Thus

$$
\phi(t)=\kappa e^{\mu_{1}(t+c \tau)}, \quad t \leq 0 .
$$

Hence, if $t>0$ and $\phi(t) \geq \kappa$ for all $t \geq 0$ (this requirement is automatically satisfied for each monotone bistable wave), then $\phi(t)$ for $t>0$ satisfies the equation

$$
\phi^{\prime \prime}(t)-c \phi^{\prime}(t)-\phi+1+q(\phi(t-c \tau)-1)=0 .
$$

The change of variables $\psi=\phi-1$ transforms this equation into

$$
\psi^{\prime \prime}(t)-c \psi^{\prime}(t)-\psi+q \psi(t-c \tau)=0 .
$$

We also have that

$$
\psi(t)=\kappa e^{\mu_{1}(t+c \tau)}-1, t \in[-c \tau, 0], \quad \psi(0)=\kappa e^{\mu_{1} c \tau}-1, \quad \psi^{\prime}(0)=\kappa \mu_{1} e^{\mu_{1} c \tau} .
$$

Applying the Laplace transform $(L \psi)(z)=\int_{0}^{\infty} e^{-z t} \psi(t) d t$ to equation (12), we get

$$
\chi(z)(L \psi)(z)=\psi^{\prime}(0)+z \psi(0)-c \psi(0)-q e^{-z c \tau} \int_{-c \tau}^{0} e^{-z t} \psi(t) d t .
$$


Here $\chi(z)=z^{2}-c z-1+q e^{-c \tau z}$ has a unique positive zero $\lambda_{1}$, see Lemma 3.2. Furthermore, we will assume that the parameters $c, \tau, q$ are such that $\lambda_{1}$ is the only zero of $\chi(z)$ on the closed right half-plane. Then the stable manifold of the zero equilibrium to (12) has codimension 1 and the solution of initial value problem (13) for this equation belongs to the stable manifold if and only if the projection of the initial function on the unstable manifold is zero, i.e. if and only if

$$
\kappa \mu_{1} e^{\mu_{1} c \tau}+\left(\lambda_{1}-c\right)\left(\kappa e^{\mu_{1} c \tau}-1\right)-q e^{-\lambda_{1} c \tau} \int_{-c \tau}^{0} e^{-\lambda_{1} t} \psi(t) d t=0 .
$$

After an integration, this gives

$$
\kappa\left(\mu_{1} e^{\mu_{1} c \tau}+\left(\lambda_{1}-c\right) e^{\mu_{1} c \tau}-q \frac{e^{\left(\mu_{1}-\lambda_{1}\right) c \tau}-1}{\mu_{1}-\lambda_{1}}\right)=\left(\lambda_{1}-c\right)+\frac{q}{\lambda_{1}}\left(e^{-\lambda_{1} c \tau}-1\right) .
$$

Since $\mu_{1}$ and $\lambda_{1}$ are solutions of equations (11) and $\chi(z)=0$, respectively, the last equation simplifies to

$$
\kappa=\frac{1-q}{p-q}\left(1-\frac{\mu_{1}(c)}{\lambda_{1}(c)}\right)=: K(c)>0, \quad c \geq 0 .
$$

Being simple zeros, $\lambda_{1}(c)$ and $\mu_{1}(c)$ are positive continuous functions of $c$ and clearly $K(+\infty)=0$. In addition, due to $(9)$,

$$
K(0)=\frac{1-q}{p-q}\left(1-\frac{\mu_{1}(0)}{\lambda_{1}(0)}\right)=\frac{1-q}{p-q}\left(1-\sqrt{\frac{1-p}{1-q}}\right)=\left(1+\sqrt{\frac{1-p}{1-q}}\right)^{-1}>\kappa .
$$

Thus for each delay $\tau \geq 0$ there exists at least one speed $c>0$ such that equation (14) is satisfied. In fact, the next result shows that such $c$ is actually unique (and therefore, for each fixed $\tau$, bistable wavefront of the 'toy' version of (6) is unique up to translation).

Lemma 2.7 It holds that $\left(\mu_{1}(c) / \lambda_{1}(c)\right)^{\prime}>0$ for all $c>0$.

Proof. For $c>0$, set $\epsilon=c^{-2}$ and observe that functions $\mu(\epsilon):=c \mu_{1}(c)$ and $\lambda(\epsilon):=c \lambda_{1}(c)$ satisfy the equations $\epsilon z^{2}-z-1+p e^{-z \tau}=0, \epsilon z^{2}-z-1+q e^{-z \tau}=0$, respectively. Clearly, the lemma statement amounts to $(\mu(\epsilon) / \lambda(\epsilon))^{\prime} \neq 0$. So, on the contrary, suppose that the latter derivative is equal to 0 at some point $\epsilon_{0}$. Then $\mu^{\prime}\left(\epsilon_{0}\right) \lambda\left(\epsilon_{0}\right)=\mu\left(\epsilon_{0}\right) \lambda^{\prime}\left(\epsilon_{0}\right)$. Set $\lambda_{0}=\lambda\left(\epsilon_{0}\right), \mu_{0}=\mu\left(\epsilon_{0}\right)$. Since

$$
\lambda^{\prime}\left(\epsilon_{0}\right)=-\frac{\lambda_{0}^{2}}{2 \epsilon_{0} \lambda_{0}-1+\tau\left(\epsilon_{0} \lambda_{0}^{2}-\lambda_{0}-1\right)}, \mu^{\prime}\left(\epsilon_{0}\right)=-\frac{\mu_{0}^{2}}{2 \epsilon_{0} \mu_{0}-1+\tau\left(\epsilon_{0} \mu_{0}^{2}-\mu_{0}-1\right)},
$$

the equality $\mu^{\prime}\left(\epsilon_{0}\right) \lambda\left(\epsilon_{0}\right)=\mu\left(\epsilon_{0}\right) \lambda^{\prime}\left(\epsilon_{0}\right)$ is equivalent to

$$
\frac{2 \epsilon_{0} \lambda_{0}-1+\tau\left(\epsilon_{0} \lambda_{0}^{2}-\lambda_{0}-1\right)}{\lambda_{0}}=\frac{2 \epsilon_{0} \mu_{0}-1+\tau\left(\epsilon_{0} \mu_{0}^{2}-\mu_{0}-1\right)}{\mu_{0}},
$$

which can be simplified to the following contradictory relations

$$
-(1+\tau)\left(\frac{1}{\lambda_{0}}-\frac{1}{\mu_{0}}\right)=\tau \epsilon_{0}\left(\mu_{0}-\lambda_{0}\right), \quad 0>-(1+\tau)=\tau \epsilon_{0} \mu_{0} \lambda_{0}>0
$$




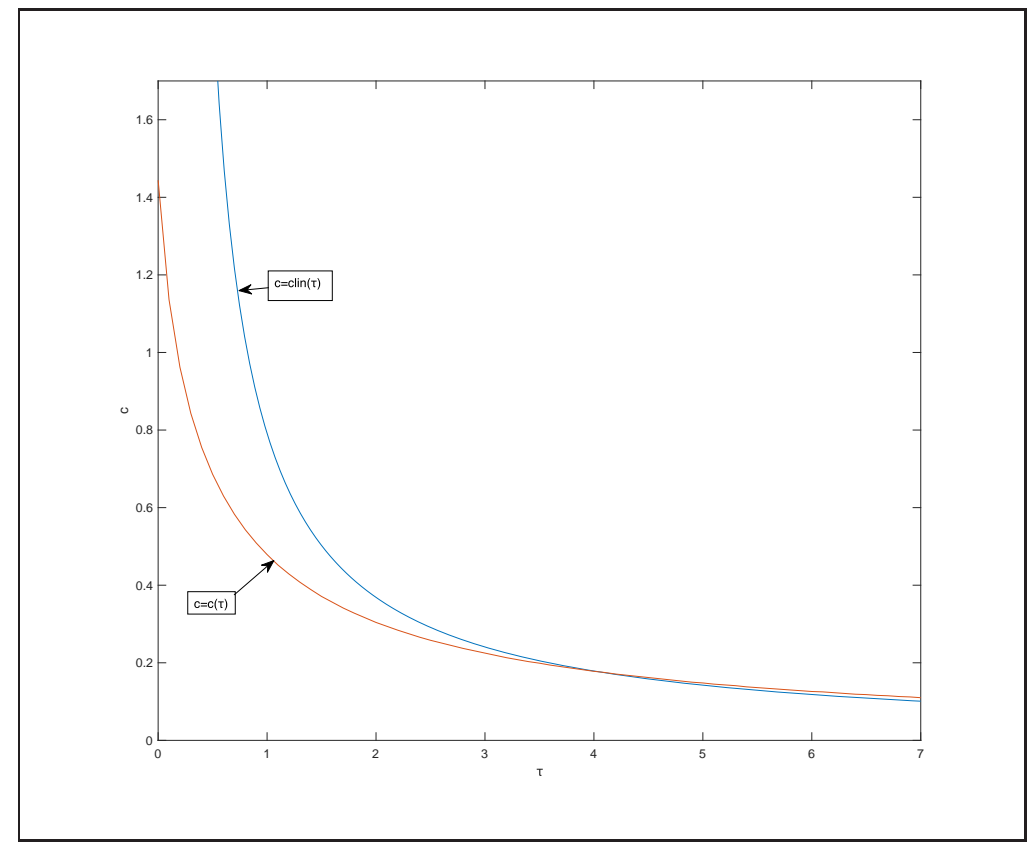

Figure 2. Domain $\mathcal{D}(-1,-1)$ and the curve $c=c(\tau)$ for the 'toy' model with $\kappa=1 / 3, p=1 / 2, q=-1$.

For numerics, we take $\kappa=1 / 3, p=1 / 2, q=-1$ as shown on Figure 1 (left). Then for small values of delays $(0 \leq \tau \leq 4.04 \ldots)$, our 'toy' equation has monotone bistable waves propagating with speeds $c=c(\tau)$, see Figure 2. However, for bigger delays (i.e. for $\tau>4.04 \ldots)$ the bistable wave profile $\phi(t)$ oscillates around the equilibrium 1 at $+\infty$. Figure 2 also suggests that $c(\tau)$ is a decreasing function of $\tau$.

If we now suppose that $k_{*}>1$ (i.e. $\int_{0}^{1}(-u+f(u)) d u<0$, see Figure 1 , right), then the propagation speed must be negative, $c<0$. Using the same notations $\lambda_{1}(|c|), \mu_{1}(|c|)$ and applying the Laplace transform approach again, we find that for every delay $\tau \geq 0$ there exists a unique monotone bistable wave propagating with the speed $c=c(\tau)<0$ which can be determined from the equation

$$
1-\kappa=\frac{1-p}{p-q}\left(\frac{\lambda_{1}(|c|)}{\mu_{1}(|c|)}-1\right)>0, \quad c<0 .
$$

For $t \geq 0$, the explicit form of the unique profile normalised by the condition $\phi(-c \tau)=\kappa$ is given by $\phi(t)=1-(1-\kappa) e^{-\lambda_{1}(|c|)(t+c \tau)}$. For numerical calculations in this case, we take $\kappa=0.9, p=1 / 2, q=-1$ as shown on Figure 1, right. Figure 3 also suggests that $|c(\tau)|$ is a decreasing function of $\tau$ and shows that the inequality $|c(\tau)|>\operatorname{clin}(\tau)$ may happen for certain values of $\tau$ (unlike the case when $c>0$, the latter does not affect the monotonicity property of the profile $\phi$ ). 


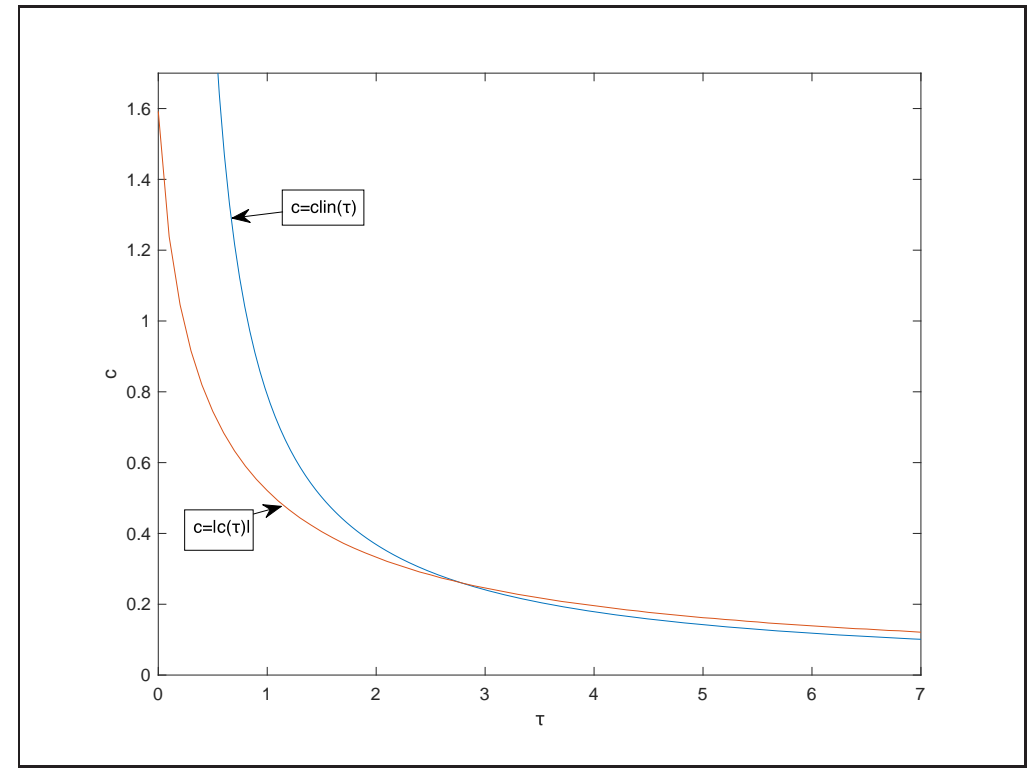

Figure 3. Domain $\mathcal{D}(-1,-1)$ and the curve $c=|c(\tau)|$ for the 'toy' model with $\kappa=0.9, p=1 / 2, q=-1$.

\section{Variational equation along the monotone bistable wave under assumption (U).}

Let $\phi(t)$ be a solution of problem (2) with $c>0$ satisfying $\phi(t) \geq e_{1}$ for all $t \in \mathbb{R}$. Without restricting generality, we can assume that $\phi(-c \tau)=\kappa$ and that $\phi(t)<\kappa$ for $t<-c \tau$. The variational equation along $\phi(t)$ is of the form $\mathcal{D} \psi(t)=0$, where

$$
\mathcal{D} \psi(t):=\psi^{\prime \prime}(t)-c \psi^{\prime}(t)+a(t) \psi(t)+b(t) \psi(t-c \tau)
$$

and

$$
a(t):=g_{1}(\phi(t), \phi(t-c \tau)), \quad b(t)=g_{2}(\phi(t), \phi(t-c \tau)) .
$$

Clearly, $\mathcal{D} \phi^{\prime}(t)=0$. In view of assumptions (B), (U), we have that $b(0)=0 ; a(t)<0$ for $t<-c \tau ; b(t)<0$ for $t<0$ and $b(t)>0$ for $t>0$;

$$
\begin{gathered}
a_{-}:=a(-\infty)=g_{1}\left(e_{1}, e_{1}\right)<0, \quad b_{-}:=b(-\infty)=g_{2}\left(e_{1}, e_{1}\right)<0 \\
a_{+}:=a(+\infty)=g_{1}\left(e_{3}, e_{3}\right)<0, \quad b_{+}:=b(+\infty)=g_{2}\left(e_{3}, e_{3}\right)>0, \quad a_{+}+b_{+}<0 .
\end{gathered}
$$

Hence, the variational equation is asymptotically autonomous and the limiting autonomous equations at $\pm \infty$ have the characteristic functions $\chi_{ \pm}(z)=z^{2}-c z+$ $a_{ \pm}+b_{ \pm} e^{-z c \tau}$. It is easy to see that $\chi_{+}(z)$ always has exactly two real roots (we will denote them by $\left.\mu_{2}<0<\mu_{1}\right)$ and that $\chi_{-}(z)$ always has exactly one positive root (we will use the notation $\lambda_{1}$ for it). Some further information about zeros of $\chi_{ \pm}(z)$ can be found in the next two lemmas.

Lemma 3.1 The zeros $\mu_{1}, \mu_{2}$ are simple. Moreover, they are unique zeros of $\chi_{+}(z)$ in the half-plane $\left\{\Re z \geq \mu_{2}\right\}$. 
Proof. Since $\chi_{+}(x)<0$ for all $x \in\left(\mu_{1}, \mu_{2}\right)$ and $\chi_{+}^{\prime \prime}(x)>0, x \in \mathbb{R}$, the equalities $\chi_{+}^{\prime}\left(\mu_{j}\right)=0, j=1,2$, are excluded. Thus $\mu_{1}, \mu_{2}$ are simple zeros. Next, let $z_{j}^{ \pm}, j=1,2$, denote the real zeros of the polynomial $z^{2}-c z+a_{ \pm}$. Then $z_{1}^{+}<\mu_{2}<0<\mu_{1}<z_{2}^{+}$. If $w$ is a complex zero of $\chi_{+}(z)$, it holds that

$$
\left|\Re w-z_{1}^{+}\right|\left|\Re w-z_{2}^{+}\right|<\left|w-z_{1}^{+}\right|\left|w-z_{2}^{+}\right|=b_{+} e^{-\Re w c \tau},
$$

so that, for each complex zero $w$ with $\Re w \in\left[\mu_{1}, \mu_{2}\right]$

$$
0<b_{+} e^{-\Re w c \tau}-\left|\Re w-z_{1}^{+}\right|\left|\Re w-z_{2}^{+}\right|=b_{+} e^{-\Re w c \tau}-\left(\Re w-z_{1}^{+}\right)\left(z_{2}^{+}-\Re w\right)=\chi_{+}(\Re w),
$$

contradicting to the inequality $\chi_{+}(x)<0, x \in\left(\mu_{1}, \mu_{2}\right)$.

Similarly, for each $z=i y, y \in \mathbb{R}$, it holds

$$
\left|b_{+} e^{-z c \tau}\right|=b_{+}<-a_{+}=\left|\Re i y-z_{1}^{+}\right|\left|\Re i y-z_{2}^{+}\right| \leq\left|z-z_{1}^{+}\right|\left|z-z_{2}^{+}\right|=\left|z^{2}-c z+a_{+}\right| .
$$

As a consequence, by a standard argument invoking the Rouché theorem, the numbers of roots of $z^{2}-c z+a_{+}$and $z^{2}-c z+a_{+}+b_{+} e^{-z c \tau}$ on the half-plane $\{\Re z \geq 0\}$ coincide (due to decaying nature of $b_{+} e^{-x c \tau}$ for $x>0$ ).

Lemma $3.2 \lambda_{1}$ is simple and dominating zero of $\chi_{-}(z)$ : every other root $\lambda_{j}$ of the equation $\chi_{-}(z)=0$ satisfies $\Re \lambda_{j}<\lambda_{1}$. If $\lambda_{2}$, $\Im \lambda_{2} \geq 0$, is a root with the biggest real part $\Re \lambda_{2}<\lambda_{1}$, then $\lambda_{2}$ is a unique root of $\chi_{-}(z)=0$ with these properties belonging to the upper half-plane. Moreover, $\lambda_{2}$ is either real negative root of the maximal multiplicity 2, or it is a simple complex root.

Proof. Clearly, $\chi_{-}^{\prime}\left(\lambda_{1}\right)>0$ and therefore the multiplicity of $\lambda_{1}$ is equal to 1 . Next, for each $z$ with $x=\Re z>\lambda_{1}$, we have that

$\left|z^{2}-c z+a_{-}\right|=\left|z-z_{1}^{-}\right|\left|z-z_{2}^{-}\right| \geq\left(x-z_{1}^{-}\right)\left(x-z_{2}^{-}\right)=x^{2}-c x+a_{-}>\left|b_{-}\right| e^{-c \tau x}=\left|b_{-} e^{-c \tau z}\right|$,

so that every zero $\lambda_{j}$ of the characteristic function should satisfy $\Re \lambda_{j} \leq \lambda_{1}$. Now, if $\Re z=\lambda_{1}, \Im z \neq 0$, then $\left|z-z_{1}^{-}\right|\left|z-z_{2}^{-}\right|>\left(x-z_{1}^{-}\right)\left(x-z_{2}^{-}\right)$so that again $\left|z^{2}-c z+a_{-}\right|>\left|e^{-c \tau z}\right|$.

Next, if $\lambda_{j}=\alpha+i \beta_{j}, 0 \leq \beta_{2}<\beta_{3}, j=2,3$, are two zero of $\chi_{-}(z)$, then

$$
\left|\lambda_{2}^{2}-c \lambda_{2}+a_{-}\right|=\left|b_{-}\right| e^{-c \tau \alpha}=\left|\lambda_{3}^{2}-c \lambda_{3}+a_{-}\right| \text {. }
$$

On the other hand,

$$
\left|\lambda_{2}^{2}-c \lambda_{2}+a_{-}\right|=\left|\lambda_{2}-z_{1}^{-}\right|\left|\lambda_{2}-z_{2}^{-}\right|<\left|\lambda_{3}-z_{1}^{-}\right|\left|\lambda_{3}-z_{2}^{-}\right|=\left|\lambda_{3}^{2}-c \lambda_{3}+a_{-}\right|,
$$

a contradiction which proves the uniqueness of $\lambda_{2}$. Let us suppose now that the complex zero $\lambda_{2}$ is multiple. Then $\chi_{-}\left(\lambda_{2}\right)=\chi_{-}^{\prime}\left(\lambda_{2}\right)=0$ that implies $\lambda_{2}^{2}-(c-$ $2 /(c \tau)) \lambda_{2}+a_{-}-1 / \tau=0$. Since the latter quadratic equation has only real roots, we get a contradiction. Finally, inequality $a_{-}<0$ implies that the system of equations 
$\chi_{-}\left(\lambda_{2}\right)=\chi_{-}^{\prime}\left(\lambda_{2}\right)=\chi_{-}^{\prime \prime}\left(\lambda_{2}\right)=0$ is incompatible. In this way, the multiplicity of $\lambda_{2}$ cannot exceed two.

Equation $\mathcal{D} \psi(t)=0$ can be written as the system

$$
v^{\prime}(t)=w(t), w^{\prime}(t)=-a(t) v(t)+c w(t)-b(t) v(t-c \tau),
$$

or shortly as $\mathfrak{F}_{c}(v, w)=0$, where

$$
\mathfrak{F}_{c}(v, w)(t)=\left(v^{\prime}(t)-w(t), w^{\prime}(t)+a(t) v(t)-c w(t)+b(t) v(t-c \tau)\right) .
$$

System (15) possesses exponential dichotomy at $+\infty$ and shifted exponential dichotomy with exponents $\alpha_{1}:=\lambda_{1}-1.5 \delta<\lambda_{1}-0.5 \delta=$ : $\beta_{1}$ (for $\delta>0$ small enough to satisfy $\left.\Re \lambda_{2}<\lambda_{1}-1.5 \delta\right)$ at $-\infty$, see [15] for the definition of these dichotomies. As a consequence, each solution of (15) converging to 0 at $+\infty$, has an exponential rate of decay. Since for each bistable wavefront $\phi$ we have that $\phi^{\prime \prime}( \pm \infty)=\phi^{\prime}( \pm \infty)=0$, we conclude that $\phi^{\prime}, \phi^{\prime \prime}$ converge to 0 at $+\infty$ with the exponential rate. More precise asymptotic formulas are given in Lemma 3.4. To deal with the problem of superexponentially small solutions in the proof of Lemma 3.4, we first establish the positivity of $e_{3}-\phi(t)$ for all $t \in \mathbb{R}$ :

Lemma 3.3 Assume (U) and suppose that $\phi(t) \geq e_{1}, t \in \mathbb{R}, \phi(-c \tau)=\kappa$, is a bistable wavefront for equation (2) propagating with the speed $c>0$. Then $\phi(t)<e_{3}, t \in \mathbb{R}$. If $\psi(t), \psi(-\infty)=e_{1}$, is a non-constant solution of equation (2) which is non-decreasing on the maximal interval $(-\infty, s]$ then $\psi(s-c \tau)>\kappa$. Consequently, if $\psi(t)$ is normalized by the relation $\psi(-c \tau)=\kappa$ then $s>0$. Moreover, the normalized solution $\psi(t)$ satisfies $\psi^{\prime \prime}(t)>0$ and $\psi^{\prime}(t)>0$ for all $t \leq 0$.

Proof. (a) Indeed, otherwise $\phi(t)$ reaches its absolute maximum on $\mathbb{R}$ at some point $s_{2}$, where $\phi^{\prime}\left(s_{2}\right)=0, \phi^{\prime \prime}\left(s_{2}\right) \leq 0, \phi\left(s_{2}\right) \geq e_{3}, \phi\left(s_{2}\right)>\phi\left(s_{2}-c \tau\right)$. However, in view of the inequalities

$$
\begin{gathered}
g\left(\phi\left(s_{2}\right), \phi\left(s_{2}-c \tau\right)\right)<g\left(\phi\left(s_{2}\right), \phi\left(s_{2}\right)\right) \leq 0, \text { if } \phi\left(s_{2}-c \tau\right) \geq \kappa ; \\
g\left(\phi\left(s_{2}\right), \phi\left(s_{2}-c \tau\right)\right)<g\left(\phi\left(s_{2}\right), e_{1}\right)<0, \text { if } e_{1}<\phi\left(s_{2}-c \tau\right)<\kappa ; \\
g\left(\phi\left(s_{2}\right), \phi\left(s_{2}-c \tau\right)\right)=g\left(\phi\left(s_{2}\right), e_{1}\right)<0, \text { if } e_{1}=\phi\left(s_{2}-c \tau\right),
\end{gathered}
$$

this contradicts to equation (2).

(b) Suppose that $\psi\left(s^{\prime}-c \tau\right) \leq \kappa$ and $\psi^{\prime}\left(s^{\prime}\right)=0$ for some $s^{\prime} \leq s$. Then $\psi^{\prime}\left(s^{\prime}\right)=0, \psi^{\prime \prime}\left(s^{\prime}\right) \leq$ 0 so that

$$
\begin{gathered}
0 \leq g\left(\psi\left(s^{\prime}\right), \psi\left(s^{\prime}-c \tau\right)\right)<g\left(\psi\left(s^{\prime}-c \tau\right), \psi\left(s^{\prime}-c \tau\right)\right) \leq 0, \text { if } \psi\left(s^{\prime}\right) \leq e_{2} \text { and } \psi\left(s^{\prime}-c \tau\right)<\psi\left(s^{\prime}\right) ; \\
0 \leq g\left(\psi\left(s^{\prime}\right), \psi\left(s^{\prime}-c \tau\right)\right)=g\left(\psi\left(s^{\prime}-c \tau\right), \psi\left(s^{\prime}-c \tau\right)\right)<0, \text { if } \psi\left(s^{\prime}\right)=\psi\left(s^{\prime}-c \tau\right) \leq \kappa ; \\
0 \leq g\left(\psi\left(s^{\prime}\right), \psi\left(s^{\prime}-c \tau\right)\right)<g\left(\psi\left(s^{\prime}\right), e_{1}\right)<0, \text { if } \psi\left(s^{\prime}\right)>e_{2},
\end{gathered}
$$

a contradiction. The same argument works if we suppose that $\psi^{\prime}\left(s^{\prime}\right) \geq 0, \psi^{\prime \prime}\left(s^{\prime}\right)=0$. 
Lemma 3.4 Assume (U) and suppose that $\phi(t) \geq e_{1}, t \in \mathbb{R}$, is a bistable wavefront for equation (2) propagating with the speed $c>0$. Then, for some appropriate $t_{1} \in \mathbb{R}$ and small $\epsilon>0$,

$$
\phi\left(t+t_{1}\right)=e_{3}-e^{\mu_{2} t}+O\left(e^{\left(\mu_{2}-\epsilon\right) t}\right), \quad \phi^{\prime}\left(t+t_{1}\right)=-\mu_{2} e^{\mu_{2} t}+O\left(e^{\left(\mu_{2}-\epsilon\right) t}\right), t \rightarrow+\infty .
$$

In particular, $\phi(t)$ is eventually strictly increasing at $+\infty$.

Proof. Recall that system (15) is exponentially dichotomic at $+\infty$. As a consequence, $\phi^{\prime}(t), \phi^{\prime \prime}(t)$ and positive function $\phi_{1}(t)=e_{3}-\phi(t)=\int_{t}^{+\infty} \phi^{\prime}(s) d s$ converge to 0 exponentially at $+\infty$. Thus, for some positive $\nu$ and $t \rightarrow+\infty$, functions

$$
a_{j}(t):=\int_{0}^{1} g_{j}\left(e_{3}-(1-s) \phi_{1}(t), e_{3}-(1-s) \phi_{1}(t-c \tau)\right) d s
$$

satisfy

$$
a_{1}(t)=a_{+}+O\left(e^{-\gamma \nu t}\right), \quad a_{2}(t)=b_{+}+O\left(e^{-\gamma \nu t}\right), t \rightarrow+\infty .
$$

Now, since positive $\phi_{1}(t)$ satisfies the equation

$$
\phi_{1}^{\prime \prime}(t)-c \phi_{1}^{\prime}(t)+a_{1}(t) \phi_{1}(t)+a_{2}(t) \phi_{1}(t-c \tau)=0,
$$

the super-exponential convergence of $\phi_{1}(t)$ to 0 as $t \rightarrow+\infty$ is not possible due to [21, Lemma 3.1.1 under Assumption 3.1.1]. Thus Proposition 7.2 from [25] implies that, for some eigenvalue $\mu_{j}, \Re \mu_{j}<0$, of $\chi_{+}(z)$, small positive $r$ and non-zero polynomials $p_{j}(t), q_{j}(t)$, it holds

$$
\left(\phi_{1}(t), \phi_{1}^{\prime}(t)\right)=\left(p_{j}(t), q_{j}(t)\right) e^{\mu_{j} t}+O\left(e^{\left(\Re \mu_{j}-r\right) t}\right), t \rightarrow+\infty .
$$

In view of the positivity of $\phi_{1}$ at $+\infty, \mu_{j}$ should be a real negative number. Thus actually $\mu_{j}=\mu_{2}$. By Lemma 3.1, $\mu_{2}$ is a simple zero of $\chi_{+}(z)$ and therefore $p_{j}>0, q_{j}=\mu_{2} p_{j}$ are constants.

In the next two lemmas, we study the asymptotic behavior of bistable wavefronts at $-\infty$.

Lemma 3.5 Assume (U) and suppose that $\phi(t)$ is profile of a bistable wavefront which is monotone at $-\infty$. Then, for some appropriate $t_{1} \in \mathbb{R}$ and small $\epsilon>0$,

$$
\phi\left(t+t_{1}\right)=e_{1}+e^{\lambda_{1} t}+O\left(e^{\left(\lambda_{1}+\epsilon\right) t}\right), \quad \phi^{\prime}\left(t+t_{1}\right)=\lambda_{1} e^{\lambda_{1} t}+O\left(e^{\left(\lambda_{1}+\epsilon\right) t}\right), t \rightarrow-\infty .
$$

In particular, $\phi^{\prime}(t)>0$ on some maximal open interval $(-\infty, s), s>0$, described in Lemma 3.3.

Proof. In view of Lemma 3.3, $\psi(t)=\phi^{\prime}(t)>0, \psi^{\prime}(t)=\phi^{\prime \prime}(t)>0$ on some maximal interval $(-\infty, s)$. Since $\mathcal{D} \psi(t)=0$, we find that $\left(\psi^{\prime}(t) e^{-c t}\right)^{\prime}>0$ because of

$$
\psi^{\prime \prime}(t)-c \psi^{\prime}(t)=-a(t) \psi(t)-b(t) \psi(t-c \tau)>0, \quad t<-c \tau .
$$


Therefore $\psi^{\prime}(t) e^{-c t}<\psi^{\prime}(s) e^{-c s}$ for $t<s<-c \tau$, or, equivalently, $\phi^{\prime \prime}(t)<\phi^{\prime \prime}(s) e^{-c(s-t)}$ for $t<s<-c \tau$. Thus $\phi^{\prime}(t)=\int_{-\infty}^{t} \phi^{\prime \prime}(s) d s, \phi^{\prime \prime}(t)$ converge to 0 exponentially at $-\infty$, so that, for some positive $\nu$,

$$
a(t)=a_{-}+O\left(e^{\nu t}\right), \quad b(t)=b_{-}+O\left(e^{\nu t}\right), \quad t \rightarrow-\infty .
$$

Applying now [21, Lemma 3.1.1 under Assumption 3.1.2], Proposition 7.2 from [25], we obtain that, for some eigenvalue $\lambda_{j}, \Re \lambda_{j}>0$, of $\chi_{-}(z)$, small positive $r$ and non-zero polynomials $p_{j}(t), q_{j}(t)$, it holds

$$
\left(\phi^{\prime}(t), \phi^{\prime \prime}(t)\right)=\left(p_{j}(t), q_{j}(t)\right) e^{\lambda_{j} t}+O\left(e^{\left(\Re \lambda_{j}+r\right) t}\right), t \rightarrow-\infty .
$$

Now, eventual monotonicity of $\phi(t)$ at $-\infty$ implies eventual non-negativity or nonpositivity of $\phi^{\prime}(t)$. Thus $\lambda_{j}$ should be a real positive number. This yields that actually $\lambda_{j}=\lambda_{1}$ and $p_{j}$ is a non-zero constant. Now, if $p_{j}$ is negative, then $\phi(t)$ is strictly decreasing at $-\infty$ and therefore there is the leftmost number $s$ such that $\phi^{\prime}(s)=0$, $\phi^{\prime \prime}(s) \geq 0, e_{1}>\phi(s-c \tau)>\phi(s)$. This contradicts, however, to equation (2):

$$
0 \geq g(\phi(s), \phi(s-c \tau))>g\left(\phi(s), e_{1}\right)>0 .
$$

In this way, $p_{j}>0$ that proves the second formula in (17) for an appropriate $t_{1}$, while the similar formula for $\phi(t)$ at $t \rightarrow-\infty$ follows from the representation $\phi(t)-e_{1}=\int_{-\infty}^{t} \phi^{\prime}(s) d s$.

The next result (used immediately afterwards, in the proof of Lemma 3.7) excludes the existence of small solutions to asymptotically autonomous delayed differential equations at $-\infty$ :

Lemma 3.6 Suppose that $L, M(t): C\left([-h, 0], \mathbb{R}^{n}\right) \rightarrow \mathbb{R}^{n}, t \leq 0$, are continuous linear operators and $\|M(t)\| \rightarrow 0$ as $t \rightarrow-\infty$ (here $\|\cdot\|$ denotes the operator norm). Then the system

$$
x^{\prime}(t)=(L+M(t)) x_{t}, x_{t}(s):=x(t+s), s \in[-h, 0],
$$

does not have exponentially small solutions at $-\infty$ (i.e. non-zero solutions $x: \mathbb{R}_{-} \rightarrow \mathbb{R}^{n}$ such that for each $\gamma \in \mathbb{R}$ it holds that $\left.x(t) e^{\gamma t} \rightarrow 0, t \rightarrow-\infty\right)$.

Proof. On the contrary, suppose that there exists a small solution $x(t)$ of $(18)$ at $-\infty$. Take some $b>0$. It is straightforward to see that the property $x(t) e^{\gamma t} \rightarrow 0, t \rightarrow-\infty$, is equivalent to $\left|x_{t}\right|_{b} e^{\gamma t} \rightarrow 0, t \rightarrow-\infty$, where $\left|x_{t}\right|_{b}=\max _{s \in[-b, 0]}|x(t+s)|$. Next, smallness of $x(t)$ implies that $\inf _{t \leq 0}\left|x_{t-b}\right|_{b} /\left|x_{t}\right|_{b}=0$. Indeed, otherwise there is $K>0$ such that $\left|x_{t-b}\right|_{b} /\left|x_{t}\right|_{b} \geq K, t \leq 0$, and therefore, setting $\nu:=b^{-1} \ln K$, we obtain the following contradiction:

$$
0<\left|x_{t}\right|_{b} e^{\nu t} \leq\left|x_{t-b}\right|_{b} e^{\nu(t-b)} \leq\left|x_{t-2 b}\right|_{b} e^{\nu(t-2 b)} \leq \ldots \leq\left|x_{t-m b}\right|_{b} e^{\nu(t-m b)} \rightarrow 0, \quad m \rightarrow+\infty .
$$

Hence, for $b=3 h$ there is a sequence $t_{j} \rightarrow-\infty$ such that $\left|x_{t_{j}-3 h}\right|_{b} /\left|x_{t_{j}}\right|_{3 h} \rightarrow 0$ as $j \rightarrow \infty$. Clearly, $\left|x_{t_{j}}\right|_{3 h}=\left|x\left(s_{j}\right)\right|$ for some $s_{j} \in\left[t_{j}-3 h, t_{j}\right]$ and, for all large $j$, it holds 
$\left|x\left(s_{j}\right)\right| \geq|x(s)|, s \in\left[t_{j}-6 h, t_{j}\right]$. Since $0 \leq t_{j}-s_{j} \leq 3 h$, without loss of generality we can assume that $\theta_{j}:=t_{j}-s_{j} \rightarrow \theta_{*} \in[0,3 h]$.

Now, for sufficiently large $j$, consider the sequence of functions

$$
y_{j}(t)=\frac{x\left(t+t_{j}\right)}{\left|x\left(s_{j}\right)\right|}, t \in[-6 h, 0], \quad\left|y_{j}\left(-\theta_{j}\right)\right|=1, \quad\left|y_{j}(t)\right| \leq 1, t \in[-6 h, 0] .
$$

For each $j, y_{j}(t)$ satisfies the equations

$$
y^{\prime}(t)=\left(L+M\left(t+s_{j}\right)\right) y_{t}, \quad y_{j}(t)=y_{j}\left(-\theta_{j}\right)+\int_{-\theta_{j}}^{t}\left(L+M\left(u+s_{j}\right)\right) y_{u} d u,
$$

and therefore $\left|y_{j}(t)\right| \leq 1,\left|y^{\prime}(t)\right| \leq\|L\|+\sup _{s \leq 0}\|M(s)\|, t \in[-5 h, 0], j \in \mathbb{N}$. Thus, due to the Arzelà-Ascoli theorem, there exists a subsequence $y_{j_{k}}(t)$ converging, uniformly on $[-5 h, 0]$, to some continuous function $y_{*}(t)$ such that $\left|y_{*}\left(-\theta_{*}\right)\right|=1$,

$$
y_{*}(t)=y_{*}\left(-\theta_{*}\right)+\int_{-\theta_{*}}^{t} L\left(y_{*}\right)_{u} d u, \quad t \in[-4 h, 0] .
$$

In particular, $y_{*}^{\prime}(t)=L\left(y_{*}\right)_{t}, t \in[-4 h, 0]$. Since $y_{*}(t)=0$ for all $t \in[-5 h,-3 h]$, the existence and uniqueness theorem applied to the initial value problem $y^{\prime}(t)=L y_{t}, t \in$ $[-4 h, 0], y_{-3 h}=0$, implies that also $y_{*}(t)=0$ for all $t \in[-3 h, 0]$. However, this contradicts the relation $\left|y_{*}(-\theta)\right|=1$. The proof of Lemma 3.6 is completed.

Lemma 3.7 Assume condition (U). If $\phi(t)$ is a solution of equation (2) such that $\phi(0)>e_{1}$ and

$$
\sup _{t \leq 0}\left|\phi(t)-e_{1}\right| e^{-\left(\lambda_{1}-\delta\right) t}<\infty
$$

for some $\delta \in\left(0, \lambda_{1}\right)$ small enough to satisfy $\Re \lambda_{2}<\lambda_{1}-1.5 \delta$, then $\phi^{\prime}(t)>0$ on the maximal open interval $(-\infty, s)$ described in Lemma 3.3.

Proof. Set $\phi_{1}(t)=e_{1}-\phi(t)$ and $\nu=\lambda_{1}-\delta$. Then $\phi_{1}(t)$ satisfies equation (16) where

$$
\begin{gathered}
a_{j}(t):=\int_{0}^{1} g_{j}\left(e_{1}-(1-s) \phi_{1}(t), e_{1}-(1-s) \phi_{1}(t-c \tau)\right) d s, \\
a_{1}(t)=a_{-}+O\left(e^{\gamma \nu t}\right), \quad a_{2}(t)=b_{-}+O\left(e^{\gamma \nu t}\right), t \rightarrow-\infty .
\end{gathered}
$$

By Lemma 3.6, $\phi_{1}(t)$ is not super-exponentially small at $-\infty$. Since $\phi_{1}^{\prime}(-\infty)=0$, we can again invoke Proposition 7.2 from [25] and Lemma 3.2 to conclude that, for some eigenvalue $\lambda_{j}, \Re \lambda_{j}>0$, of $\chi_{-}(z)$, small positive $r$ and non-zero constants $p_{j}, q_{j}$, it holds that

$$
\left(\phi_{1}(t), \phi_{1}^{\prime}(t)\right)=\left(p_{j}, q_{j}\right) e^{\lambda_{j} t}+O\left(e^{\left(\Re \lambda_{j}+r\right) t}\right), t \rightarrow-\infty .
$$

But then, in the latter case, condition (19) implies that $\lambda_{j}=\lambda_{1}$. This leads to the conclusion of the lemma, see the final part of the proof of Lemma 3.5.

In the remainder of this section, we are assuming that the profile $\phi(t)$ of bistable wave is strictly increasing, i.e. $\phi^{\prime}(t)>0, t \in \mathbb{R}$. After fixing some $\delta \in\left(0,0.5\left(\lambda_{1}-\right.\right.$ 
$\left.\left.\Re \lambda_{2}\right)\right) \cap\left(0, \lambda_{1}\right) \cap\left(0,-\mu_{2}\right)$, such that $(1+\gamma)\left(\mu_{2}+\delta\right)<\mu_{2}$, we will consider $\mathfrak{F}_{c}$ as a linear operator defined on $C_{\delta}^{1}$ and taking its values in $C_{\delta}$, where

$$
\begin{gathered}
C_{\delta}=\left\{y=\left(y_{1}, y_{2}\right) \in C\left(\mathbb{R}, \mathbb{R}^{2}\right):|y|_{\delta}:=\sup _{s \leq 0} e^{-\left(\lambda_{1}-\delta\right) s}|y(s)|+\sup _{s \geq 0} e^{-\left(\mu_{2}+\delta\right) s}|y(s)|<\infty\right\}, \\
C_{\delta}^{1}=\left\{y \in C_{\delta}: y, y^{\prime} \in C_{\delta},|y|_{1, \delta}:=|y|_{\delta}+\left|y^{\prime}\right|_{\delta}<+\infty\right\} .
\end{gathered}
$$

Remark 3.8 From Lemmas 3.4 and 3.5 we obtain that $\left(\phi^{\prime}, \phi^{\prime \prime}\right) \in C_{\delta}$ and that $a(t)=$ $a_{+}+O\left(e^{\gamma \mu_{2} t}\right), b(t)=b_{+}+O\left(e^{\gamma \mu_{2} t}\right), \quad t \rightarrow+\infty$.

The system which is formally adjoint [16] to (15) has the following form

$$
v^{\prime}(t)=a(t) w(t)+b(t+c \tau) w(t+c \tau), \quad w^{\prime}(t)=-v(t)-c w(t) .
$$

This amounts to the following equation for $w(t)$ :

$$
\mathcal{D}^{*} w(t):=w^{\prime \prime}(t)+c w^{\prime}(t)+a(t) w(t)+b(t+c \tau) w(t+c \tau)=0
$$

The following result is obvious.

Lemma 3.9 Suppose that functions $a(t), b(t)$ are continuous and $b(t) \neq 0$ if $t \neq 0$. If $\mathcal{D}^{*} w(t)=0$ and, for some $t^{\prime}$, it holds that either $w(t)=0$ for all $t \leq t^{\prime}$ or $w(t)=0$ for all $t \geq t^{\prime}$, then $w(t) \equiv 0$.

The equation $\mathcal{D}^{*} w(t)=0$ with advanced argument can be transformed in the usual delayed equation by means of the transformation $w(t) \rightarrow w(-t)$ :

$$
w^{\prime \prime}(t)-c w^{\prime}(t)+a(-t) w(t)+b(-t+c \tau) w(t-c \tau)=0 .
$$

A description of the leading zeros of the characteristic functions $\chi_{ \pm}(z)$ provided in Lemmas 3.1, 3.2 together with an analysis of the asymptotic properties of wavefronts realized in Lemmas 3.3, 3.4, 3.5, 3.7 and Remark 3.8 enable us to establish the Fredholm properties of operators $\mathcal{D}, \mathfrak{F}_{c}$. Note that Fredholmness of asymptotically autonomous functional differential operators (respectively, of delayed, of mixed or of non-local type and considered in different spaces) was studied by Hale and Lin [15], Mallet-Paret [25] and in $[3,9,30]$. In fact, the next result can be deduced from any of these works:

Proposition $3.10 \mathfrak{F}_{c}: C_{\delta}^{1} \rightarrow C_{\delta}$ is Fredholm operator of index ind $\mathfrak{F}_{c}=0$. Moreover, $\mathfrak{F}_{c}$ has one-dimensional kernel $N\left(\mathfrak{F}_{c}\right)=<\left(\phi^{\prime}(t), \phi^{\prime \prime}(t)\right)>$. Thus range $R\left(\mathfrak{F}_{c}\right)$ of $\mathfrak{F}_{c}$ has codimension 1 and therefore

$$
R\left(\mathfrak{F}_{c}\right)=\left\{F=\left(f_{1}, f_{2}\right) \in C_{\delta}: \int_{\mathbb{R}}\left(f_{1}(s) v_{*}(s)+f_{2}(s) w_{*}(s)\right) d s=0\right\},
$$

where $z(t)=\left(v_{*}(t), w_{*}(t)\right)$ is the unique (up to a constant multiple) non-zero solution of (20) satisfying inequalities

$$
|z(t)| \leq K e^{-\left(\mu_{1}-0.5 \delta\right) t}, t \geq 0 ; \quad|z(t)| \leq K e^{-\left(\lambda_{1}-1.5 \delta\right) t}, t \leq 0 .
$$


Proof. Due to our choice of spaces, it is convenient to use the theory developed in [15]. Particularly, we are going to show how Proposition 3.10 can be deduced from Lemmas 4.6 and 4.5 in [15]. In order to simplify the use of these lemmas and to show their relation to similar results in $[3,9,25]$, for $(p, q)=\left(\lambda_{1}-\delta, \mu_{2}+\delta\right), q \leq 0 \leq p$, we consider $C^{\infty}$-smooth weight function $\mu: \mathbb{R} \rightarrow[1,+\infty)$ such that $\mu(t)=e^{-p t}, t \leq-1$ and $\mu(t)=e^{-q t}, t \geq 1$. Following [15], we introduce the notation

$$
\begin{aligned}
& C(p, q)=\left\{y=\left(y_{1}, y_{2}\right) \in C\left(\mathbb{R}, \mathbb{R}^{2}\right):|y|_{p, q}:=\sup _{s \in \mathbb{R}} \mu(s)|y(s)|<\infty\right\}, \\
& C^{1}(p, q)=\left\{y \in C(p, q): y, y^{\prime} \in C(p, q),|y|_{1, p, q}:=|y|_{p, q}+\left|y^{\prime}\right|_{p, q}<+\infty\right\} .
\end{aligned}
$$

Clearly, $C_{\delta}=C\left(\lambda_{1}-\delta, \mu_{2}+\delta\right), C_{\delta}^{1}=C^{1}\left(\lambda_{1}-\delta, \mu_{2}+\delta\right)$ and the multiplication operator $L y=\mu y$,

$$
L: C^{1}(p, q) \rightarrow C^{1}(0,0), \quad L: C(p, q) \rightarrow C(0,0),
$$

is an isomorphism of the Banach spaces. Consider $B:=L \mathfrak{F}_{c} L^{-1}: C^{1}(0,0) \rightarrow C(0,0)$,

$$
\begin{aligned}
& B(v, w)(t)=\left(v^{\prime}(t)-\frac{\mu^{\prime}(t)}{\mu(t)} v(t)-w(t),\right. \\
& \left.w^{\prime}(t)-\frac{\mu^{\prime}(t)}{\mu(t)} w(t)+a(t) v(t)-c w(t)+b(t) \frac{\mu(t)}{\mu(t-c \tau)} v(t-c \tau)\right) .
\end{aligned}
$$

Since the limiting equations $B_{ \pm \infty}(v, w)(t)=0$ at $-\infty$ and $+\infty$,

$$
\begin{aligned}
& B_{-}(v, w)(t)=\left(v^{\prime}(t)+p v(t)-w(t), w^{\prime}(t)+p w(t)+a_{-} v(t)-c w(t)+b_{-} e^{-p c \tau} v(t-c \tau)\right) . \\
& B_{+}(v, w)(t)=\left(v^{\prime}(t)+q v(t)-w(t), w^{\prime}(t)+q w(t)+a_{-} v(t)-c w(t)+b_{-} e^{-q c \tau} v(t-c \tau)\right),
\end{aligned}
$$

have the characteristic functions $\chi_{-}(z+p)=\chi_{-}\left(z+\lambda_{1}-\delta\right), \chi_{+}(z+q)=\chi_{+}\left(z+\mu_{2}+\delta\right)$, they both are exponentially dichotomic with one-dimensional unstable spaces, cf. Lemma 3.1,3.2. Then Lemmas 4.6 and 4.5 in [15] imply that $B$ (and, consequently, $\left.\mathfrak{F}_{c}=L^{-1} B L\right)$ is Fredholm of index 0 . The same conclusion can be obtained by using approach proposed in [9, Theorems 2.2 and 3.2]. Next, it is clear (see also [15, Lemma 4.6]) that dimension of kernel $N(B)$ of $B$ cannot exceed the dimension of the unstable space of $B_{-}$, i.e $\operatorname{dim} N(B) \leq 1$. On the other hand, by Remark 3.8, we have that $L\left(\phi^{\prime}, \phi^{\prime \prime}\right)=\mu\left(\phi^{\prime}, \phi^{\prime \prime}\right) \in C(0,0) \cap N(B)$ so that $\operatorname{dim} N(B)=\operatorname{dim} N\left(\mathfrak{F}_{c}\right)=\operatorname{codim} R\left(\mathfrak{F}_{c}\right)=$ $\operatorname{codim} R(B)=1$.

Finally, [15, Lemma 4.5] (or, equivalently, [25, Theorem A and Proposition 5.3]) implies that $R(B)=\left\{F \in C(0,0): \int_{\mathbb{R}} F(s) y_{*}(s) d s=0\right\}$, where $y_{*}(s)$ is an exponentially decaying non-zero solution of the formally adjoint equation $B^{*} y=0$,

$$
\begin{aligned}
& B^{*}(v, w)(t)=\left(v^{\prime}(t)+\frac{\mu^{\prime}(t)}{\mu(t)} v(t)-a(t) w(t)-b(t+c \tau) \frac{\mu(t+c \tau)}{\mu(t)} w(t+c \tau),\right. \\
& \left.w^{\prime}(t)+\frac{\mu^{\prime}(t)}{\mu(t)} w(t)+v(t)+c w(t)\right) .
\end{aligned}
$$

The characteristic functions of the limiting equations for $B^{*}$ at $-\infty$ and $+\infty$ are, respectively, $\chi_{-}(p-z)=\chi_{-}\left(\lambda_{1}-\delta-z\right), \chi_{+}(q-z)=\chi_{+}\left(\mu_{2}+\delta-z\right)$. Therefore, 
from [25, Proposition 7.2$]$ we find that $y_{*}(t)=O\left(t e^{\left(\lambda_{1}-\lambda_{2}-\delta\right) t}\right)$ at $-\infty$, and $y_{*}(t)=$ $O\left(e^{\left(\mu_{2}-\mu_{1}+\delta\right) t}\right), t \rightarrow+\infty$. Thus

$R\left(\mathfrak{F}_{c}\right)=L^{-1} R(B)=\left\{F \in C_{\delta}: \int_{\mathbb{R}} F(s) \mu(s) y_{*}(s) d s=0\right\}=\left\{F \in C_{\delta}: \int_{\mathbb{R}} F(s) z(s) d s=0\right\}$,

where $z(s):=\mu(s) y_{*}(s)$ clearly satisfies the estimates of the proposition as well as equation (20); the latter can be verified by a direct calculation.

Remark 3.11 In view of Lemma 3.9, we can assume that $w_{*}(s)>0$ for some $s>0$. Next, set $w(t)=w_{*}(-t)$. Then $w^{\prime}(t)=-w_{*}^{\prime}(-t)=v_{*}(-t)+c w_{*}(-t)$ so that

$$
\left|\left(w(t), w^{\prime}(t)\right)\right|=O\left(e^{\left(\mu_{1}-0.5 \delta\right) t}\right), t \leq 0 ; \quad\left|\left(w(t), w^{\prime}(t)\right)\right|=O\left(e^{\left(\lambda_{1}-1.5 \delta\right) t}\right), t \geq 0 .
$$

Since the characteristic function of the limiting equation for (21) at $+\infty$ is $\chi_{-}(z)$, Proposition 7.2 in [25] guarantees that either $w(t)$ is super-exponentially small at $+\infty$ or, for some eigenvalue $\lambda_{j}$ with the real part $\Re \lambda_{j} \leq \Re \lambda_{2}$ and for some polynomial $P(t)$ of the degree less than or equal to one, it holds

$$
w(t)=P(t) e^{\Re \lambda_{j} t} \cos \left(\Im \lambda_{j} t+\phi\right)+O\left(e^{\left(\Re \lambda_{j}-\varepsilon\right) t}\right), t \rightarrow+\infty .
$$

In particular, this shows that either $w_{*}(t)$ is exponentially (or even super-exponentially) small at $-\infty$ or $w_{*}(t)$ is non-decaying and oscillating around 0 at $-\infty$.

Corollary 3.12 Set $Y_{\delta}:=\left\{y \in C(\mathbb{R}, \mathbb{R}):(y, y) \in C_{\delta}\right\}$ and $X_{\delta}:=\left\{y \in C^{2}(\mathbb{R}, \mathbb{R})\right.$ : $\left.y, y^{\prime}, y^{\prime \prime} \in Y_{\delta}\right\}$, then $\mathcal{D}: X_{\delta} \rightarrow Y_{\delta}$ is continuous Fredholm operator of index 0 and one-dimensional kernel $N(\mathcal{D})=<\phi^{\prime}(t)>$. The range $R(\mathcal{D})$ of $\mathcal{D}$ is given by

$$
R(\mathcal{D})=\left\{f \in Y_{\delta}: \int_{\mathbb{R}} f(s) w_{*}(s) d s=0\right\},
$$

where $w_{*}(t)$ is described in Proposition 3.10.

Proof. Indeed, $y \in N(\mathcal{D})$ if and only if $\left(y, y^{\prime}\right) \in N\left(\mathfrak{F}_{c}\right)$. Similarly, $f \in R(\mathcal{D})$ if and only if $(0, f) \in R\left(\mathfrak{F}_{c}\right)$, i.e. if and only if $\int_{\mathbb{R}} f(s) w_{*}(s) d s=0$.

Lemma 3.13 The solution $w_{*}$ is positive on $(0,+\infty): w_{*}(t)>0, t>0$.

Proof. To prove the non-negativity of $w_{*}(t)$ on $\mathbb{R}_{+}$, we are going to use, similarly to the proofs of [31, Theorem 5.1] or [20, Theorem 2.5], an appropriate test function $f$. Recall that, by our assumption (see Remark 3.11), $w_{*}(s)>0$ for some $s>0$.

Claim 1. It holds that $w_{*}(t) \geq 0$ for all $t \in \mathbb{R}_{+}$.

Indeed, otherwise we can indicate a function $f \in Y_{\delta}$ and a real number $T>0$ with the following properties

(i) $f(t)=0, t \leq 0$, and $f(t)<0, t>0$;

(ii) $f(t)=\mathcal{D}\left(t e^{\mu_{2} t}\right)=e^{\mu_{2} t}\left(\chi_{+}^{\prime}\left(\mu_{2}\right)+o(1)\right)<0, t \geq T$;

(iii) $f(t)$ is smooth on $\mathbb{R}_{+}$and $f^{\prime}(0+)<0$; 
(iv) $\int_{\mathbb{R}} f(t) w_{*}(t) d t=0$.

Then, by Corollary 3.12, the inhomogeneous equation

$$
\psi^{\prime \prime}(t)-c \psi^{\prime}(t)+a(t) \psi(t)+b(t) \psi(t-c \tau)=f(t)
$$

has a solution $\psi_{*} \in X_{\delta}$. Since $f(t)=0$ for $t \leq 0$, we conclude that there exists $T_{2}>0$ such that the vector $\left(\psi_{*}(t), \psi_{*}^{\prime}(t)\right), t \leq-T_{2}$, belong to the unstable space of the system $\mathfrak{F}_{c}(v, w)=0, t \leq-T_{2}$ which has a shifted exponential dichotomy at $-\infty$. Now, since the exponents $\alpha_{1}:=\lambda_{1}-1.5 \delta<\lambda_{1}-0.5 \delta=: \beta_{1}<\lambda_{2}$ of the shifted exponential dichotomy satisfy $\Re \lambda_{2}<\alpha_{1}<\beta_{1}<\lambda_{1}$, this unstable space has dimension 1 and therefore $\psi_{*}(t)=k \phi^{\prime}(t), t \leq-T_{2}$, for some $k>0$. For certain, this yields immediately that $\psi_{*}(t)=k \phi^{\prime}(t), t \leq 0$.

On the other hand, due to our definition of $f(t)$ for positive $t$, we obtain that the function $q(t):=\psi_{*}(t)-t e^{\mu_{2} t}, t \geq T+c \tau$ satisfies the homogeneous equation $\mathcal{D} q(t)=0, t \geq T+c \tau$. Since $q(t), q^{\prime}(t)$ have an exponential rate of convergence to 0 at $+\infty$, we can apply Proposition 7.2 from [25] together with Remark 3.8, to conclude that $q(t)=O\left(e^{\mu_{2} t}\right), t \rightarrow+\infty$. This shows that the solution $\psi(t, \xi)=\psi_{*}(t)+\xi \phi^{\prime}(t)$ of $(23)$ is positive at $+\infty$ for every real $\xi$. Since also $\psi(t, \xi)=(k+\xi) \phi^{\prime}(t), t \leq 0$, we obtain that $\psi(t, \xi)>0, t \in \mathbb{R}$, for all large $\xi>0$. Let now $\xi_{*}:=\inf \{\xi: \psi(t, \xi)>0, t \in \mathbb{R}\}$. Clearly, $\xi_{*}$ is finite and $\psi(t, \xi) \geq 0, t \in \mathbb{R}$, if and only if $\xi \geq \xi_{*}$. Next, we have that either $\psi\left(t, \xi_{*}\right)>0$ for $t \leq 0$ or $\psi\left(t, \xi_{*}\right) \equiv 0$ on $\mathbb{R}_{-}$. In the first case, $\psi\left(t_{*}, \xi_{*}\right)=0$ for some $t_{*}>0$ (since otherwise $\psi\left(t, \xi_{*}-\epsilon\right)>0, t \in \mathbb{R}$, for all small $\epsilon>0$ ). However, this implies that $\psi^{\prime \prime}\left(t_{*}, \xi_{*}\right) \geq \psi^{\prime}\left(t_{*}, \xi_{*}\right)=0$ contradicting to $(23)$ at $t_{*}$ (since $f\left(t_{*}\right)<0, b\left(t_{*}\right)>0$ and $\left.\psi\left(t_{*}-c \tau, \xi_{*}\right) \geq 0\right)$. In the second case, $C^{2}$-smooth function $\psi\left(t, \xi_{*}\right)$ satisfies on $[0, c \tau]$ the following ordinary equation with zero initial data:

$$
\psi^{\prime \prime}(t)-c \psi^{\prime}(t)+a(t) \psi(t)=f(t), \psi(0)=\psi^{\prime}(0)=0 .
$$

In particular, $\psi^{\prime \prime}\left(0, \xi_{*}\right)=0$. However, $\psi^{\prime \prime \prime}\left(0+, \xi_{*}\right)=f^{\prime}(0+)<0$ and therefore $\psi\left(t, \xi_{*}\right)<0$ for all small positive $t$, contradicting to the definition of $\xi_{*}$. This completes the proof of Claim 1.

Claim 2. It holds that $w_{*}(t)>0$ for all $t>0$.

To analyse the asymptotical behaviour of $w_{*}(t)$ for positive $t$, it is convenient to consider $\hat{w}(t)=w_{*}(-t)$. This function satisfies the delayed equation (21) which is asymptotically autonomous at $-\infty$, with the limiting equation

$$
w^{\prime \prime}(t)-c w^{\prime}(t)+a_{+} w(t)+b_{+} w(t-c \tau)=0 .
$$

Since $|\hat{w}(t)| \leq K e^{\left(\mu_{1}-0.5 \delta\right) t}, t \leq 0$, and by Remark $3.8 a(-t)-a_{+}, b(-t+c \tau)-b_{+}$are exponentially small at $-\infty$, we deduce, as before, from [25, Proposition 7.2] and Lemma 3.6 the following asymptotic representation

$$
\hat{w}(t)=d e^{\mu_{1} t}+O\left(e^{\left(\mu_{1}+\epsilon\right) t}\right), \quad t \rightarrow-\infty,
$$

with some positive $\epsilon, d$. Consequently, $w_{*}(t)>0$ on some interval $(m,+\infty)$. Let $m$ be the leftmost point for which the inequality $w_{*}(t)>0, t \in(m,+\infty)$ holds. If $m>0$, then 
$w_{*}(m)=w_{*}^{\prime}(m)=0 \leq w_{*}^{\prime \prime}(m)$. Therefore, in view of equation $\mathcal{D}^{*} w_{*}(t)=0$, we find that $b(m+c \tau) w(m+c \tau) \leq 0$, contradicting to the fact that $b(m+c \tau)>0, w(m+c \tau)>0$.

Remark 3.14 The second example considered in Subsection 2.3 (with $k_{*}>1$ and $c<0$ ) shows that, in general, $w_{*}(t)$ can oscillates on $(-\infty, 0)$. On the other hand, we believe that $w_{*}(t)>0, t \in \mathbb{R}$, if $|c(\tau)|<\operatorname{clin}(\tau)$, cf. Fig.3. See the next section where we prove such a kind of result when $\left(\mathbf{U}^{*}\right)$ is assumed instead of $(\mathbf{U})$.

\section{Variational equation along the monotone bistable wave, case of hypotheses $(\mathrm{B}),\left(\mathrm{U}^{*}\right)$.}

Let profile $\phi(t)$ of the bistable wavefront for problem (2) considered with $c>0$ be such that $\phi^{\prime}(t)>0, t \in \mathbb{R}$. Again, we can assume that $\phi(-c \tau)=\kappa$ and that $\phi(t)<\kappa$ for $t<-c \tau$. In view of assumptions (B), ( $\left.\mathbf{U}^{*}\right)$, the coefficients $a(t), b(t)$ of the differential operator $\mathcal{D}$ satisfy the relations $b(0)=0, b(t)>0$ for $t<0$ and $a(t), b(t)<0$ for $t>0$, while

$$
\begin{gathered}
a_{+}:=a(-\infty)=g_{1}\left(e_{1}, e_{1}\right)<0, \quad b_{+}:=b(-\infty)=g_{2}\left(e_{1}, e_{1}\right)>0 ; \quad b_{+}+a_{+}<0 ; \\
a_{-}:=a(+\infty)=g_{1}\left(e_{3}, e_{3}\right)<0, \quad b_{-}:=b(+\infty)=g_{2}\left(e_{3}, e_{3}\right)<0 .
\end{gathered}
$$

Hence, the variational equation is asymptotically autonomous and the limiting autonomous equations at $\pm \infty$ have the characteristic functions $\chi_{\mp}(z)$. Clearly, above convention on the notation allows the application of Lemmas 3.1 and 3.2 describing properties of zeros of $\chi_{\mp}(z)$. In Lemma 4.1 below, we show how the monotonicity of wavefront $\phi(t)$ at $+\infty$ propagating with speed $c$ implies that $\chi_{-}(z)$ has exactly two (counting the multiplicity) real negative zeros $\lambda_{3} \leq \lambda_{2}<0$ (i.e. implying that $(\tau, c) \in \mathfrak{D})$. Therefore $\phi^{\prime}(t)$ decays at $+\infty$ with the exponential rate which is asymptotically equivalent to $p(t) \exp \left(\lambda_{j} t\right)$, where $j \in\{2,3\}$ and $p(t)$ is a polynomial. Our approach, however, requires slowest possible decay of $\phi^{\prime}(t)$ at $+\infty$. We are reaching this goal assuming the sub-tangency condition at the steady state $e_{3}$ in the hypothesis $\left(\mathbf{U}^{*}\right)$. As we show in Lemma 4.1, this condition forces $\phi^{\prime}(t)$ to have the required asymptotical behavior at $+\infty$. It is worth to mention that the slowest decay of $\phi^{\prime}(t)$ at $+\infty$ was automatically assured in the case of the hypothesis $(\mathbf{U})$. This explains why a similar sub-tangency condition was not required in $(\mathbf{U})$.

Lemma 4.1 Let the hypotheses (B), ( $\left.\mathbf{U}^{*}\right)$ be satisfied. If equation (6) has a nondecreasing bistable wavefront and $\chi_{-}(z)$ does not have roots on the imaginary axis, then $\chi_{-}(z)$ has exactly two negative zeros (counting multiplicity) $\lambda_{3} \leq \lambda_{2}$. Moreover, for some appropriate $t_{1} \in \mathbb{R}, A>0, j \in\{0,1\}$, and small $\epsilon>0$,

$\phi\left(t+t_{1}\right)=e_{3}-A t^{j} e^{\lambda_{2} t}+o\left(t^{j} e^{\lambda_{2} t}\right), \phi^{\prime}\left(t+t_{1}\right)=-A \lambda_{2} t^{j} e^{\lambda_{2} t}+o\left(t^{j} e^{\lambda_{2} t}\right), t \rightarrow+\infty$.

Here $j=1$ if and only if $\lambda_{2}=\lambda_{3}$. 
Proof. Since $\chi_{-}(z)$ does not have roots on the imaginary axis, system (15) is exponentially dichotomic at $+\infty$. As a consequence, $\phi^{\prime}(t), \phi^{\prime \prime}(t)$ converge to 0 exponentially fast at $+\infty$. Thus, for some positive $\nu$,

$$
a(t)=a_{-}+O\left(e^{-\nu t}\right), \quad b(t)=b_{-}+O\left(e^{-\nu t}\right), \quad t \rightarrow+\infty .
$$

Applying now [21, Lemma 3.1.1 under Assumption 3.1.2], Proposition 7.2 from [25], we obtain that, for some eigenvalue $\lambda_{j}, \Re \lambda_{j}<0$, of $\chi_{-}(z)$, small positive $r$ and non-zero polynomials $p_{j}(t), q_{j}(t)$, it holds that

$$
\left(\phi^{\prime}(t), \phi^{\prime \prime}(t)\right)=\left(p_{j}(t), q_{j}(t)\right) e^{\lambda_{j} t}+O\left(e^{\left(\Re \lambda_{j}-r\right) t}\right), t \rightarrow+\infty .
$$

Now, monotonicity of $\phi(t)$ at $+\infty$ implies non-negativity of $\phi^{\prime}(t)$. Thus $\lambda_{j}$ should be a real negative number. This yields that actually $\lambda_{j} \in\left\{\lambda_{2}, \lambda_{3}\right\}$ and $p_{j}$ is a positive constant (if $\lambda_{3}<\lambda_{2}$ ) or at most first order non-zero polynomial (if $\lambda_{2}=\lambda_{3}$ ). The similar formula for $\phi(t)$ at $t \rightarrow+\infty$ follows from the equality $\phi(t)-e_{3}=-\int_{t}^{+\infty} \phi^{\prime}(s) d s$.

In order to prove that $j=2$ in the case when $g$ is sub-tangential at $e_{3}$, we observe that function $y(t):=\phi(t)-e_{3}$ satisfies the equation

$$
y^{\prime \prime}(t)-c y^{\prime}(t)+a_{-} y(t)+b_{-} y(t-c \tau)=h(t), \quad t \in \mathbb{R},
$$

where $h(t)=a_{-}\left(\phi(t)-e_{3}\right)+b_{-}\left(\phi(t-c \tau)-e_{3}\right)-g(\phi(t), \phi(t-c \tau)) \geq 0$ because of the assumed sub-tangency of $g$. Furthermore, $h(t) \not \equiv 0$ since otherwise $y(t)$ should be equal to 0 , as a unique bounded solution of the exponentially dichotomic equation. Since $g \in C^{1, \gamma}$, we also obtain that $h(t)=O\left(t^{1+\gamma} e^{(1+\gamma) \lambda_{j} t}\right)$ at $t=+\infty$. Therefore, applying the bilateral Laplace transform approach to equation (26), we find that, for some small positive $r>0$, it holds

$$
y(t)=\operatorname{Res}_{z=\lambda_{2}} \frac{e^{z t} \tilde{h}(z)}{\chi_{-}(z)}+O\left(e^{\left(\lambda_{2}-r\right) t}\right), \quad t \rightarrow+\infty .
$$

Here $\tilde{h}(z)=\int_{\mathbb{R}} e^{-z s} h(s) d s, \Re z \in\left((1+\gamma) \lambda_{2}, 0\right)$, is the bilateral Laplace transform of $h(t)$. A simple calculation shows that

$$
\begin{gathered}
\operatorname{Res}_{z=\lambda_{2}} \frac{e^{z t} \tilde{h}(z)}{\chi_{-}(z)}=\frac{e^{\lambda_{2} t} \tilde{h}\left(\lambda_{2}\right)}{\chi_{-}^{\prime}\left(\lambda_{2}\right)}=-A e^{\lambda_{2} t}, A:=-\frac{\int_{\mathbb{R}} e^{-\lambda_{2} s} h(s) d s}{\chi_{-}^{\prime}\left(\lambda_{2}\right)}>0, \quad \text { if } \lambda_{3}<\lambda_{2} ; \\
\operatorname{Res}_{z=\lambda_{2}} \frac{e^{z t} \tilde{h}(z)}{\chi_{-}(z)}=-(B t+D) e^{\lambda_{2} t}, B:=-\frac{2 \int_{\mathbb{R}} e^{-\lambda_{2} s} h(s) d s}{\chi_{-}^{\prime \prime}\left(\lambda_{2}\right)}>0, \quad \text { if } \quad \lambda_{3}=\lambda_{2} .
\end{gathered}
$$

This completes the proof of the lemma.

Remark 4.2 If we take $(\tau, c) \in \mathfrak{D}\left(\tilde{a}_{-}, \tilde{b}_{-}\right) \subset \mathfrak{D}\left(a_{-}, b_{-}\right)$where $\tilde{a}_{-}, \tilde{b}_{-}$were defined in (8), then the proof of Lemma 4.1 works even without the sub-tangency condition. Indeed, $(\tau, c) \in \mathfrak{D}\left(\tilde{a}_{-}, \tilde{b}_{-}\right)$implies that $\chi_{-}(z)$ has exactly two negative zeros (counting multiplicity) $\lambda_{3} \leq \lambda_{2}$. Thus we obtain the following assertion. 
Lemma 4.3 Let the hypotheses (B), ( $\left.\mathbf{U}^{*}\right)$ (without the sub-tangency condition) be satisfied and $(\tau, c) \in \mathfrak{D}\left(\tilde{a}_{-}, \tilde{b}_{-}\right)$. If equation (6) has a non-decreasing bistable wavefront, then for some appropriate $t_{1} \in \mathbb{R}, A>0, j \in\{0,1\}$, and small $\epsilon>0$, the representation (24) is valid.

Proof. First, suppose that, given $(\tau, c) \in \mathfrak{D}\left(\tilde{a}_{-}, \tilde{b}_{-}\right)$, we have that $\lambda_{3}=\lambda_{2}$. Since this equality can occur only for $(\tau, c)$ on the boundary of domain $\mathfrak{D}\left(a_{-}, b_{-}\right)$, we conclude that $\left(\tilde{a}_{-}, \tilde{b}_{-}\right)=\left(a_{-}, b_{-}\right)$. Since this situation was already analyzed in Lemma 4.1, we have to consider the case $\lambda_{3}<\lambda_{2}$ only. Consequently, if the formula (24) does not hold, then it should be replaced with

$$
\phi\left(t+t_{1}\right)=e_{3}-e^{\lambda_{3} t}+o\left(e^{\lambda_{3} t}\right), \quad \phi^{\prime}\left(t+t_{1}\right)=-\lambda_{3} e^{\lambda_{3} t}+o\left(e^{\lambda_{3} t}\right), t \rightarrow+\infty .
$$

Then replacing in equation (26) $\left(a_{-}, b_{-}\right)$with $\left(\tilde{a}_{-}, \tilde{b}_{-}\right)$and arguing as below (26), we find that $h(t)=O\left(e^{\lambda_{3} t}\right)$ at $t=+\infty$. Applying the bilateral Laplace transform method again, we obtain then that $\phi(t)=e_{3}-(A t+B) e^{\tilde{\lambda}_{2} t}(1+o(1)), t \rightarrow+\infty$, where $A, B$ satisfy $|A|+|B|>0$ and $\tilde{\lambda}_{2} \in\left(\lambda_{3}, \lambda_{2}\right)$ is the largest negative root of the equation $z^{2}-c z+\tilde{a}_{-}+\tilde{b}_{-} e^{-z \tau c}=0$. The latter asymptotic formula for $\phi(t)$ is however incompatible with (27).

Next, the behaviour of a bistable wavefront at $-\infty$ is described in the following proposition:

Lemma 4.4 Let the hypotheses $(\mathbf{B}),\left(\mathbf{U}^{*}\right)$ be satisfied. If $\phi(t)$ is a bistable wavefront, then there exists a maximal interval $(-\infty, m)$ such that $\phi^{\prime}(t)>0, \phi^{\prime \prime}(t)>0$ for all $t<m$. Moreover, $\phi(m) \geq e_{2}$ and, for some appropriate $t_{1} \in \mathbb{R}$ and small $\epsilon>0$,

$$
\phi\left(t+t_{1}\right)=e_{1}+e^{\mu_{1} t}+O\left(e^{\left(\mu_{1}+\epsilon\right) t}\right), \quad \phi^{\prime}\left(t+t_{1}\right)=\mu_{1} e^{\mu_{1} t}+O\left(e^{\left(\mu_{1}+\epsilon\right) t}\right), t \rightarrow-\infty .
$$

Proof. Set $\phi_{1}(t)=\phi(t)-e_{1}$ and

$$
a_{j}(t):=\int_{0}^{1} g_{j}\left(e_{1}+s \phi_{1}(t), e_{1}+s \phi_{1}(t-c \tau)\right) d s .
$$

Clearly, $\phi_{1}(-\infty)=0, \phi_{1}^{\prime}(-\infty)=0$ and $a_{1}(-\infty)=a_{+}, a_{2}(-\infty)=b_{+}$so that, in view of the properties of $\chi_{+}(z)$ established in Lemma 3.1, the differential equation for $\phi_{1}(t)$,

$$
\phi^{\prime \prime}(t)-c \phi^{\prime}(t)+a_{1}(t) \phi(t)+a_{2}(t) \phi(t-c \tau)=0,
$$

is exponentially dichotomic at $-\infty$. Moreover, this equation has one-dimensional unstable space which asymptotically converges to one-dimensional unstable space of the limit equation

$$
\phi^{\prime \prime}(t)-c \phi^{\prime}(t)+a_{+} \phi(t)+b_{+} \phi(t-c \tau)=0,
$$

see [15, Lemma 4.3]. This means that $\phi_{1}^{\prime}(t)=\left(\mu_{1}+o(1)\right) \phi_{1}(t), t \rightarrow-\infty$, and therefore for some $C \neq 0$, it holds $\phi_{1}(t)=C \exp \left(\mu_{1} t(1+o(1))\right), t \rightarrow-\infty$. If we suppose that $C<0$ than $\phi_{1}^{\prime}(t)<0$ on some maximal interval $(-\infty, s)$ where $s$ is such that $\phi(s)<e_{1}, \phi(s)<\phi(s-c \tau)<0, \phi^{\prime \prime}(s) \geq 0, \phi^{\prime}(s)=0$. Consequently, 
$g(\phi(s), \phi(s-c \tau)) \leq 0$, in contradiction with $g(\phi(s), \phi(s-c \tau))>g(\phi(s), \phi(s))>0$. Hence, $C>0$ and $\phi^{\prime}(t)>0$ on some maximal interval $(-\infty, r)$. Suppose that $r$ is finite and $\phi(r)<e_{2}$. Since, in addition, $\phi(r)>\phi(r-c \tau)>e_{1}, \phi^{\prime \prime}(r) \leq 0, \phi^{\prime}(r)=0$, we obtain that $g(\phi(r), \phi(r-c \tau)) \geq 0$, in contradiction with $g(\phi(r), \phi(r-c \tau))<$ $g(\phi(r), \phi(r))<0$. The same argument shows that the case $\phi^{\prime \prime}\left(r^{\prime}\right)=0, \phi^{\prime}\left(r^{\prime}\right)>0$ for some $r^{\prime}<r$ is not possible as well. Finally, we note that the formulas (28) is a refinement of the representation $\phi_{1}(t)=C \exp \left(\mu_{1} t(1+o(1))\right), t \rightarrow-\infty$. Since $a_{1}(t)=a_{+}+O\left(e^{\nu t}\right), \quad a_{2}(t)=b_{+}+O\left(e^{\nu t}\right), t \rightarrow-\infty$, for some positive $\nu$, they can be deduced from [25, Proposition 7.2 ], cf. the proof of Lemma 3.4.

In the remainder of this section, we assume that $(\tau, c) \in \mathfrak{D}$ and that the bistable wavefront $\phi$ is monotone. After fixing some $\delta \in\left(0, \mu_{1}\right) \cap\left(0,-\lambda_{2}\right)$ such that $(1+\gamma)\left(\mu_{2}+\delta\right)<\mu_{2}$, we will consider $\mathfrak{F}_{c}$ as a linear operator defined on $C_{\delta}^{1}$ and taking its values in $C_{\delta}$, where

$$
\begin{gathered}
C_{\delta}=\left\{y=\left(y_{1}, y_{2}\right) \in C\left(\mathbb{R}, \mathbb{R}^{2}\right):|y|_{\delta}:=\sup _{s \leq 0} e^{-\left(\mu_{1}-\delta\right) s}|y(s)|+\sup _{s \geq 0} e^{-\left(\lambda_{2}+\delta\right) s}|y(s)|<\infty\right\}, \\
C_{\delta}^{1}=\left\{y \in C_{\delta}: y, y^{\prime} \in C_{\delta},|y|_{1, \delta}:=|y|_{\delta}+\left|y^{\prime}\right|_{\delta}<+\infty\right\} .
\end{gathered}
$$

The following result is an immediate consequence of Lemmas 4.1, 4.4.

Corollary 4.5 Let the hypotheses $(\mathbf{B}),\left(\mathbf{U}^{*}\right)$ be satisfied. If $\phi(t)$ is a monotone bistable wavefront, then $\left(\phi^{\prime}, \phi^{\prime \prime}\right) \in C_{\delta}$ and $a(t)=a_{+}+O\left(e^{\gamma \mu_{1} t}\right), \quad b(t)=b_{+}+O\left(e^{\gamma \mu_{1} t}\right), t \rightarrow-\infty$.

By repeating the proof of Proposition 3.10 with $(p, q)=\left(\mu_{1}-\delta, \lambda_{2}+\delta\right)$, we conclude that

Proposition 4.6 $\mathfrak{F}_{c}: C_{\delta}^{1} \rightarrow C_{\delta}$ is Fredholm operator of index ind $\mathfrak{F}_{c}=0$. Moreover, $\mathfrak{F}_{c}$ has one-dimensional kernel $N\left(\mathfrak{F}_{c}\right)=<\left(\phi^{\prime}(t), \phi^{\prime \prime}(t)\right)>$. Thus range $R\left(\mathfrak{F}_{c}\right)$ of $\mathfrak{F}_{c}$ has codimension 1 and therefore

$$
R\left(\mathfrak{F}_{c}\right)=\left\{F=\left(f_{1}, f_{2}\right) \in C_{\delta}: \int_{\mathbb{R}}\left(f_{1}(s) v_{*}(s)+f_{2}(s) w_{*}(s)\right) d s=0\right\},
$$

where $z(t)=\left(v_{*}(t), w_{*}(t)\right)$ is the unique (up to a constant multiple) non-zero solution of (20) satisfying inequalities $|z(t)| \leq K e^{-\left(\lambda_{2}+\delta\right) t}, t \geq 0 ; \quad|z(t)| \leq K e^{-\left(\mu_{1}-\delta\right) t}, t \leq 0$.

Remark 4.7 The asymptotic estimates of $z(t)$ given in Proposition 4.6 can be easily improved till

$$
z(t)=O\left(e^{-\lambda_{1} t}\right), t \geq 0 ; \quad z(t)=O\left(e^{-\mu_{2} t}\right), t \leq 0 .
$$

For instance, let us prove the first of these formulas. Indeed, by Lemma 3.9 we can assume that $w_{*}(s)>0$ for some $s>0$. Set $w(t)=w_{*}(-t)$ then $w^{\prime}(t)=v_{*}(-t)+c w_{*}(-t)$ and thus

$$
\left|\left(w(t), w^{\prime}(t)\right)\right| \leq K_{1} e^{\left(\lambda_{2}+\delta\right) t}, t \leq 0 ; \quad\left|\left(w(t), w^{\prime}(t)\right)\right| \leq K_{1} e^{\left(\mu_{1}-\delta\right) t}, t \geq 0 .
$$


Since the characteristic function of the limiting equation for (21) at $-\infty$ is $\chi_{-}(z)$, [25, Proposition 7.2] together with Lemma 3.6 yield, with for some small $\varepsilon>0$, the following representation (possibly, after an appropriate translation of $w(t)$ )

$$
w(t)=e^{\lambda_{1} t}+O\left(e^{\left(\lambda_{1}+\varepsilon\right) t}\right), \quad w^{\prime}(t)=\lambda_{1} e^{\lambda_{1} t}+O\left(e^{\left(\lambda_{1}+\varepsilon\right) t}\right), \quad t \rightarrow-\infty .
$$

Therefore $\left(w_{*}(t), w_{*}^{\prime}(t)\right)=\left(e^{-\lambda_{1} t},-\lambda_{1} e^{-\lambda_{1} t}\right)+O\left(e^{-\left(\lambda_{1}+\varepsilon\right) t}\right), t \rightarrow+\infty$, so that $z(t)=$ $O\left(e^{-\lambda_{1} t}\right), t \geq 0$.

In addition, we obtain that $w_{*}^{\prime}(t)<0$ for all sufficiently large $t$. Let $d$ be the rightmost critical point of $w_{*}(t)$. Then $w_{*}^{\prime \prime}(d) \leq 0, w_{*}(d)>0, w_{*}(d+c \tau)>0$, so that equation $w_{*}^{\prime \prime}(d)+c w_{*}^{\prime}(d)+a(d) w_{*}(d)+b(d+c \tau) w_{*}(d+c \tau)=0$ implies that $d<0$ and $w_{*}^{\prime \prime}(t)>0$ for all $t \geq 0$.

Lemma 4.8 Let the hypotheses $(\mathbf{B}),\left(\mathbf{U}^{*}\right)$ be satisfied. Then solution $w_{*}(t)$ is positive for $t \geq 0$ and non-negative for $t \leq 0: w_{*}(t) \geq 0, t \in \mathbb{R}_{-}$.

Proof. As we have already established in Remark 4.7, $w_{*}(t)>0$ for all $t \geq 0$. Suppose for a moment that $w_{*}(t)$ takes negative values on $(-\infty, 0)$. Then there are a function $f \in Y_{\delta}$ and a real number $T>0$ with the following properties

(i) $f(t)=0, t \geq 0$, and $f(t)<0, t<0$;

(ii) $\left.f(t)=\mathcal{D}\left(-t e^{\mu_{1} t}\right)=-e^{\mu_{1} t}\left(\chi_{+}^{\prime}\left(\mu_{1}\right)+o(1)\right)\right)<0, t \leq-T$, (Corollary 4.5 is used here);

(iii) $\int_{\mathbb{R}} f(t) w_{*}(t) d t=0$.

Then, by Corollary 3.12, the inhomogeneous equation

$$
\psi^{\prime \prime}(t)-c \psi^{\prime}(t)+a(t) \psi(t)+b(t) \psi(t-c \tau)=f(t)
$$

has a solution $\psi_{*} \in X_{\delta}$. Since $f(t)=0$ for $t \geq 0$, and $\psi_{*}(t)$ is bounded, we conclude that $\psi_{*}(t) / \phi^{\prime}(t)$ converges to a finite limit as $t \rightarrow+\infty$.

On the other hand, due to our definition of $f(t)$ for negative $t$, we obtain that the function $q(t):=\psi_{*}(t)+t e^{\mu_{1} t}, t \leq-T$, satisfies the homogeneous equation $\mathcal{D} q(t)=0, t \leq-T$. Since $q(t), q^{\prime}(t)$ have an exponential rate of convergence to 0 at $-\infty$, we can conclude that $q(t)=B \phi^{\prime}(t)$ for some finite $B$. This shows that the solution $\psi(t, \xi)=\psi_{*}(t)+\xi \phi^{\prime}(t)$ of $(29)$ is positive at $-\infty$ for every real $\xi$. In this way, $\psi(t, \xi)>0, t \in \mathbb{R}$, for all large $\xi>0$. Set now

$$
\xi_{*}:=\inf \{\xi: \psi(t, \xi)>0, t \in \mathbb{R}\} .
$$

Clearly, $\xi_{*}$ is finite and $\psi(t, \xi) \geq 0, t \in \mathbb{R}$, if and only if $\xi \geq \xi_{*}$. Next, since $\psi\left(t, \xi_{*}\right)$ can not have positive maxima on $\mathbb{R}_{+}$, we obtain that either $(\mathrm{A}) \psi\left(t, \xi_{*}\right)>0, \psi^{\prime}\left(t, \xi_{*}\right)<0$ for $t \geq 0$ or $(\mathrm{B}) \psi\left(t, \xi_{*}\right) \equiv 0$ on $\mathbb{R}_{+}$.

In the case $(\mathrm{B}),(29)$ implies that $\psi\left(s, \xi_{*}\right)=0, s \in[-c \tau, 0]$, so that $C^{2}$-smooth function $\psi\left(t, \xi_{*}\right)$ satisfies the following algebraic equation

$$
b(s) \psi\left(s-c \tau, \xi_{*}\right)=f(s), s \in[-c \tau, 0] .
$$


However, this is not possible because $f(s)<0$ and $b(s)>0, \psi\left(s-c \tau, \xi_{*}\right) \geq 0$ for all $s<0$.

Now, in the case (A), we have that $\psi\left(t, \xi_{*}\right)>0$ for all $t \in \mathbb{R}$. Indeed, if $\psi\left(t_{*}, \xi_{*}\right)=0$ for some $t_{*}<0$ then $\psi^{\prime \prime}\left(t_{*}, \xi_{*}\right) \geq \psi^{\prime}\left(t_{*}, \xi_{*}\right)=0$ contradicting to (29) at $t_{*}$ (since $f\left(t_{*}\right)<0, b\left(t_{*}\right)>0$ and $\left.\psi\left(t_{*}-c \tau, \xi_{*}\right) \geq 0\right)$. Next, $\psi\left(t, \xi_{*}\right)$ satisfies the differential equation

$$
\psi^{\prime \prime}(t)-c \psi^{\prime}(t)+a_{-} \psi(t)+b_{-} \psi(t-c \tau)=n(t), \quad t \in \mathbb{R},
$$

where $n(t)=m(t)+f(t)$,

$m(t):=\left[g_{1}\left(e_{3}, e_{3}\right)-g_{1}(\phi(t), \phi(t-c \tau))\right] \psi\left(t, \xi_{*}\right)+\left[g_{2}\left(e_{3}, e_{3}\right)-g_{2}(\phi(t), \phi(t-c \tau))\right] \psi\left(t-c \tau, \xi_{*}\right)$

is such that, for some small $\delta_{0}>0$, it holds

$m(t) \leq 0, t \in \mathbb{R} ; n(t)<0, t<0 ; n(t)=o(1), t \rightarrow-\infty ; n(t)=O\left(e^{\left(\lambda_{2}-\delta_{0}\right) t}\right), t \rightarrow+\infty$.

Note that the non-positivity of $m(t)$ follows from the sub-tangency assumption of ( $\left.\mathbf{U}^{*}\right)$. Applying the bilateral Laplace transform to (30) (similarly as it was done in the proof of Lemma 4.1), we find that, for some $r \in\left(0, \delta_{0}\right)$, it holds

$$
\psi\left(t, \xi_{*}\right)=\operatorname{Res}_{z=\lambda_{2}} \frac{e^{z t} \tilde{n}(z)}{\chi_{-}(z)}+O\left(e^{\left(\lambda_{2}-r\right) t}\right), \quad t \rightarrow+\infty
$$

Here $\tilde{n}(z)=\int_{\mathbb{R}} e^{-z s} n(s) d s, \Re z \in\left(\lambda_{2}-\delta_{0}, 0\right)$, is the bilateral Laplace transform of $n(t)$. A simple calculation shows that

$$
\begin{gathered}
\operatorname{Res}_{z=\lambda_{2}} \frac{e^{z t} \tilde{n}(z)}{\chi_{-}(z)}=\frac{e^{\lambda_{2} t} \tilde{n}\left(\lambda_{2}\right)}{\chi_{-}^{\prime}\left(\lambda_{2}\right)}=A e^{\lambda_{2} t}, A:=\frac{\int_{\mathbb{R}} e^{-\lambda_{2} s} n(s) d s}{\chi_{-}^{\prime}\left(\lambda_{2}\right)}>0, \quad \text { if } \lambda_{3}<\lambda_{2} ; \\
\operatorname{Res}_{z=\lambda_{2}} \frac{e^{z t} \tilde{n}(z)}{\chi_{-}(z)}=(B t+D) e^{\lambda_{2} t}, B:=\frac{2 \int_{\mathbb{R}} e^{-\lambda_{2} s} n(s) d s}{\chi_{-}^{\prime \prime}\left(\lambda_{2}\right)}>0, \quad \text { if } \quad \lambda_{3}=\lambda_{2} .
\end{gathered}
$$

The described asymptotic behaviour of $\psi\left(t, \xi_{*}\right)>0$ at $\pm \infty$ implies that $\psi\left(t, \xi_{*}-\epsilon\right)>0$, $t \in \mathbb{R}$, for all small $\epsilon>0$. However, this contradicts the definition of $\xi_{*}$. Hence, the non-negativity of $w_{*}(t)$ on $\mathbb{R}_{-}$is proved.

Remark 4.9 If, in addition to (B), ( $\left.\mathbf{U}^{*}\right)$, we assume that $g_{1}(u, v)<0$ for all $(u, v)$ satisfying $u \geq v, u \geq \kappa$, then $w_{*}(t)>0$ for all $t \in \mathbb{R}$. Indeed, in such a case, $a(t)<0$ for all $t \geq-c \tau$. By arguing as in the last paragraph of Remark 4.7, this allows to conclude that $w_{*}^{\prime \prime}(t)>0$ for all $t>-c \tau$. Let now $m$ be the leftmost point for which the inequality $w_{*}(t)>0, t \in(m,+\infty)$ holds. Clearly, $m<-c \tau$. If $m$ is finite, then $w_{*}(m)=w_{*}^{\prime}(m)=0 \leq w_{*}^{\prime \prime}(m)$. Therefore, in view of equation $\mathcal{D}^{*} w_{*}(t)=0$, we find that $b(m+c \tau) w_{*}(m+c \tau) \leq 0$, contradicting to the fact that $b(m+c \tau)>0, w_{*}(m+c \tau)>0$.

Remark 4.10 As in Remark 4.2 and Lemma 4.3, the assumption $(\tau, c) \in \mathfrak{D}\left(\tilde{a}_{-}, \tilde{b}_{-}\right) \subset$ $\mathfrak{D}\left(a_{-}, b_{-}\right)$can be used instead of the sub-tangency condition of Lemma 4.8. Indeed, 
similarly to the proof of Lemma 4.3, it suffices to replace $\left(a_{-}, b_{-}\right)$with $\left(\tilde{a}_{-}, \tilde{b}_{-}\right)$in formula (30), and, assuming that $\lambda_{3}<\lambda_{2}$,

$$
\psi\left(t, \xi_{*}\right)=A e^{\lambda_{3} t}+o\left(e^{\lambda_{3} t}\right), \quad \psi^{\prime}\left(t, \xi_{*}\right)=A \lambda_{3} e^{\lambda_{3} t}+o\left(e^{\lambda_{3} t}\right), t \rightarrow+\infty,
$$

obtain the conflicting representation $\psi\left(t, \xi_{*}\right)=(P t+Q) e^{\tilde{\lambda}_{2} t}(1+o(1)), t \rightarrow+\infty, \quad|P|+$ $|Q|>0$, with $\tilde{\lambda}_{2} \in\left(\lambda_{3}, \lambda_{2}\right)$ being the biggest negative root of the equation $z^{2}-c z+\tilde{a}_{-}+$ $\tilde{b}_{-} e^{-z \tau c}=0$.

\section{Proofs of Theorems 1.3 and 1.4 .}

In equation (2), it is convenient to use new independent parameters $c, h=c \tau$ instead of $c>0, \tau \geq 0$. Then (2) takes the form

$$
\phi^{\prime \prime}(t)-c \phi^{\prime}(t)+g(\phi(t), \phi(t-h))=0, \quad t \in \mathbb{R}, \quad \phi(-\infty)=e_{1}, \phi(+\infty)=e_{3} .
$$

\subsection{Local boundedness of the functions $c(h)$ and $c(\tau)$.}

In the coordinates $(c, h)$, the critical curve $c=\operatorname{clin}(\tau)$ and the domain $\mathfrak{D}\left(a_{-}, b_{-}\right)$have different shapes described in the following proposition. Recall that $\mathfrak{D}\left(a_{-}, b_{-}\right)$is defined as the set of non-negative parameters for which $\chi_{-}(z), c>0$, has exactly three real zeros (counting multiplicity).

Lemma 5.1 Set $h_{*}=\theta\left(a_{-}, b_{-}\right)>0$. Then there exists a continuous function $c^{\mathfrak{E}}: \mathbb{R}_{+} \rightarrow \mathbb{R}_{+}$, with the properties $c^{\mathfrak{E}}(h)=0, h \in\left[0, h_{*}\right] ; c^{\mathfrak{E}}(h)>0, h>h_{*}$, and $\lim _{h \rightarrow \infty} c^{\mathfrak{E}}(h) / h=1 / \tau_{\#}$, such that

$$
\mathfrak{D}\left(a_{-}, b_{-}\right)=\left\{(h, c): h \geq 0, c \geq c^{\mathfrak{E}}(h)\right\} \subset \mathbb{R}_{+}^{2} .
$$

Proof. Since $a_{-}<0$, it suffices to consider equation (5) for a fixed $h^{\prime} \geq 0$. Since $\mathfrak{A}\left(c, h^{\prime}\right)$ is strictly increasing to $+\infty$ with respect to $c \geq 0$ and $\mathfrak{B}\left(c, h^{\prime}\right)$ is decreasing with respect to $c \geq 0$, this equation have a unique positive solution $c^{\mathfrak{E}}\left(h^{\prime}\right)$ if and only if $\mathfrak{A}\left(0, h^{\prime}\right) \leq \mathfrak{B}\left(0, h^{\prime}\right)$. Now, it can be easily verified that the equation $\mathfrak{A}(0, h)=\mathfrak{B}(0, h)$ has a unique root $h_{*}=\theta\left(a_{-}, b_{-}\right)>0$. The computation of the $\operatorname{limit}_{h \rightarrow \infty} c^{\mathfrak{E}}(h) / h$ is immediate from equation (5) and the definition of $\tau_{\#}$ given in Remark 1.2.

Next, we show that the velocities of bistable wavefronts are uniformly bounded with respect to $h$ taken from a compact subset of $\mathbb{R}_{+}$:

Lemma 5.2 Suppose that hypothesis (B) is satisfied. Suppose further that, for each pair $\left(c_{j}, h_{j}\right)$ of parameters $h_{j} \in\left[0, h^{\prime}\right], c_{j}>0, j \in \mathbb{N}$, problem (31) has a monotone solution $\phi_{j}: \mathbb{R} \rightarrow\left[e_{1}, e_{3}\right]$. Then there exists $K=K\left(h^{\prime}\right)>0$ such that $c_{j} \leq K\left(h^{\prime}\right), j \in \mathbb{N}$.

Proof. Indeed, suppose that $\epsilon_{j}=1 / c_{j} \rightarrow 0$. After realising the change of variables $\phi_{j}(t)=\psi_{j}\left(\epsilon_{j} t\right)$ and setting $G_{j}(t)=\psi(t)+g\left(\psi(t), \psi\left(t-\epsilon_{j} h_{j}\right)\right)$, we find that $\psi_{j}(t)$ satisfies the equation

$$
\epsilon_{j}^{2} \psi^{\prime \prime}(t)-\psi^{\prime}(t)-\psi(t)=-G_{j}(t), \psi(-\infty)=e_{1}, \psi(+\infty)=e_{3} .
$$


Equation (32) is translation invariant and therefore we can suppose that $\psi_{j}(0)=$ $\left(e_{1}+e_{2}\right) / 2$. Since $\psi_{j}(t)$ is a bounded solution of (32), it satisfies the integral equation

$$
\psi_{j}(t)=\frac{1}{\sqrt{1+4 \epsilon_{j}^{2}}}\left\{\int_{-\infty}^{t} e^{z_{j}^{-}(t-s)} G_{j}(s) d s+\int_{t}^{+\infty} e^{z_{j}^{+}(t-s)} G_{j}(s) d s\right\} .
$$

where $z_{j}^{-}<0<z_{j}^{+}$denote the roots $\epsilon_{j}^{2} z^{2}-z-1=0$. Clearly, $z_{j}^{-} \rightarrow-1, z_{j}^{+} \rightarrow+\infty$. Differentiating (33), we get

$$
\psi_{j}^{\prime}(t)=\frac{1}{\sqrt{1+4 \epsilon_{j}^{2}}}\left\{z_{j}^{-} \int_{-\infty}^{t} e^{z_{j}^{-}(t-s)} G_{j}(s) d s+z_{j}^{+} \int_{t}^{+\infty} e^{z_{j}^{+}(t-s)} G_{j}(s) d s\right\} .
$$

From (34) we deduce the uniform boundedness of $\psi_{j}^{\prime}$ :

$$
\left|\psi_{j}^{\prime}(t)\right| \leq 2 \max \left\{u+|g(u, v)|, u, v \in\left[e_{1}, e_{3}\right]\right\} .
$$

Thus we can find a subsequence $\psi_{j_{k}}(t)$ of $\psi_{j}(t)$ which converges, uniformly on compact subsets of $\mathbb{R}$, to some continuous monotone function $\psi_{*}: \mathbb{R} \rightarrow\left[e_{1}, e_{3}\right]$ such that $\psi_{*}(0)=\left(e_{1}+e_{2}\right) / 2, \psi_{*}(t) \leq \psi_{*}(0)$ for $t \leq 0$. Invoking the Lebesgue dominated convergence theorem, we find that $\psi_{*}(t)$ satisfies the integral equation

$$
\psi_{*}(t)=\int_{-\infty}^{t} e^{-(t-s)}\left(\psi_{*}(s)+g\left(\psi_{*}(s), \psi_{*}(s)\right)\right) d s .
$$

In this way,

$$
\psi_{*}^{\prime}(t)=g\left(\psi_{*}(t), \psi_{*}(t)\right), \quad \psi_{*}(0)=\left(e_{1}+e_{2}\right) / 2, \psi_{*}(t) \leq \psi_{*}(0) \text { for all } t \leq 0 .
$$

However, due to the bistability of $g(x, x)$ the latter situation is not possible.

A similar result also holds for equation (2):

Lemma 5.3 Suppose that either the hypothesis (U) or $\left(\mathbf{U}^{*}\right)$ is satisfied. Then for each A there exists $K(A)>0$ such that $c(\tau) \in(0, K(A)]$ for each monotone bistable wavefront $u=\phi(x+c(\tau) t)$ of equation (1) considered with $\tau \in[0, A]$.

Proof. We have to prove that the function $c(\tau)$ is bounded on $[0, A]$. We can argue as in the proof of Lemma 5.2 with the following difference in our reasoning: now we should admit the possibility that the sequence $\tau_{j}:=\epsilon_{j} h_{j} \in[0, A]$ can posses a subsequence (we will keep the same notation $\tau_{j}$ for it) converging to a positive limit $\hat{\tau} \in[0, A]$. Similarly, we will establish the existence of a continuous monotone function $\psi_{*}: \mathbb{R} \rightarrow\left[e_{1}, e_{3}\right]$ such that $\psi_{*}(0)=\left(e_{1}+e_{2}\right) / 2, \psi_{*}(t) \leq \psi_{*}(0), t \leq 0$, and

$$
\psi_{*}^{\prime}(t)=g\left(\psi_{*}(t), \psi_{*}(t-\hat{\tau})\right) .
$$

Monotonicity and boundedness of $\psi_{*}(t)$ also implies that $\psi_{*}(-\infty)=e_{1}$ and that $\psi_{*}(+\infty) \in\left\{e_{2}, e_{3}\right\}$.

In particular, there exists $T^{\prime}$ such that $\psi_{*}(t) \in\left(e_{1}, \kappa\right]$ for all $t \leq T^{\prime}$. Therefore, if $(\mathbf{U})$ is assumed then $\psi_{*}^{\prime}(t)=g\left(\psi_{*}(t), \psi_{*}(t-\hat{\tau})\right)<0, t \leq T^{\prime}$. Since $\psi_{*}(t)$ is monotone increasing non-constant function, this leads to a contradiction. 
On the other hand, if $\left(\mathbf{U}^{*}\right)$ is assumed then the characteristic equation for linearisation of equation (35) around the equilibrium $e_{1}$ is of the form $\lambda+\left|a_{+}\right|=b_{+} e^{-\lambda \hat{\tau}}$ with $\left|a_{+}\right|>b_{+}>0$. Clearly, all roots of this equation have negative real parts so that the steady state $e_{1}$ of equation (35) is uniformly asymptotically stable. However, this is not possible due to the existence of the solution $\psi_{*}(t)$ belonging to the unstable manifold of the equilibrium $e_{1}$.

All the above said proves that the set $\{c(\tau): \tau \in[0, A]\}$ is bounded.

\subsection{Local continuation of wavefronts under assumption (U).}

Assume (U) and suppose that, given $\tau_{0} \geq 0$, equation (2) has a monotone bistable wavefront $u(t, x)=\phi_{0}\left(t+c_{0} t\right), c_{0}>0$. By Lemma 3.13, the solution $w_{*}(t)$ of equation $\mathcal{D}^{*} w(t)=0$ is non-negative on some maximal interval $[T,+\infty)$ with $T \in[-\infty, 0]$. For our considerations in this section, the case when $T$ is a finite number is much more difficult than the case $T=-\infty$. Therefore, in what follows, we assume that $T \in(-\infty, 0]$ (so that $w_{*}(T)=0$ ). If $T=-\infty$ then our subsequent arguments simplify with correctors $\psi_{\varepsilon}, S_{\varepsilon}$ (which are defined below the next lemma) taken identically zero: $\psi_{\varepsilon}(t)=S_{\varepsilon}(t)=0$ for all $t \in \mathbb{R}$ (in Section 5.3, these simplifications appear explicitly). In particular, the following result is needed only when $T \in \mathbb{R}$ :

Lemma 5.4 Set $h_{0}=c_{0} \tau_{0}$ and let $\tilde{X}_{\delta}$ and $\tilde{Y}_{\delta}$ denote the Banach spaces obtained from $X_{\delta}$ and $Y_{\delta}$ by restricting the domain of functions in $X_{\delta}, Y_{\delta}$ from $\mathbb{R}$ to $(-\infty, T]$. Then for $c, h$ close to $c_{0}, h_{0}$, equation (31) has a family of solutions $\psi_{*}(t, c, h), t \leq T+h$, with the following properties:

1) $\psi_{*}(T, c, h)=\phi_{0}(T)$ and $\psi_{*}(t, c, h)>0, \psi_{*}^{\prime}(t, c, h)>0$ for all $t \leq T$;

$\psi_{*}\left(t, h_{0}, c_{0}\right)=\phi_{0}(t)$

3) $\psi_{*}(t, c, h)$ depends $C^{1}$-smoothly on $c, h$ and $\left(\psi_{*}\right)_{c}=D_{c} \psi_{*}:=\partial \psi_{*}(\cdot, c, h) / \partial c \in \tilde{X}_{\delta}$. In particular, $\left(\psi_{*}^{\prime}\right)_{c}=\left(\left(\psi_{*}\right)_{c}\right)^{\prime},\left(\left(\psi_{*}^{\prime}\right)_{c}\right)^{\prime}=\left(\left(\psi_{*}\right)_{c}\right)^{\prime \prime} \in \tilde{Y}_{\delta}$.

Proof. We can consider solution $\psi_{*}(t, c, h)$ as a perturbation of $\phi_{0}(t)$ :

$$
\psi_{*}(t, c, h)=\phi_{0}(t)+\zeta(t, c, h), \quad \zeta \in \tilde{X}_{\delta} .
$$

Then the equation for $\zeta$ is $\mathcal{D}_{0} \zeta(t)=N(\zeta, c, h)(t)$, where

$$
\begin{gathered}
\mathcal{D}_{0} \zeta(t):=\zeta^{\prime \prime}(t)-c_{0} \zeta^{\prime}(t)+a(t) \zeta(t)+b(t) \zeta\left(t-h_{0}\right), \\
N(\zeta, c, h)=\left(c-c_{0}\right)\left(\phi_{0}^{\prime}(t)+\zeta^{\prime}(t)\right)+a(t) \zeta(t)+b(t) \zeta\left(t-h_{0}\right)+ \\
+g\left(\phi_{0}(t), \phi_{0}\left(t-h_{0}\right)\right)-g\left(\phi_{0}(t)+\zeta(t), \phi_{0}(t-h)+\zeta(t-h)\right), \quad N\left(0, c_{0}, h_{0}\right)=0 .
\end{gathered}
$$

An auxiliary technical result given below, Lemma 5.8, implies that $N: \tilde{X}_{\delta} \times(0, \infty) \times$ $[0, \infty) \rightarrow \tilde{Y}_{\delta}$ is continuously differentiable and $D_{\zeta} N\left(0, c_{0}, h_{0}\right)=0$. On the other hand, as it was shown in Claim I of Lemma 3.13, continuous linear operator $\mathcal{D}_{0}: \tilde{X}_{\delta} \rightarrow \tilde{Y}_{\delta}$ has one-dimensional kernel: $\operatorname{dim} \operatorname{Ker} \mathcal{D}_{0}=1$. We claim that, in addition, $\mathcal{D}_{0}$ is a surjective operator. Indeed, take some $f \in \tilde{Y}_{\delta}$ and set Set $f_{1}(t):=f(t) e^{-\left(\lambda_{1}-\delta\right) t}$. Then 
consider inhomogeneous equation $\mathcal{D}_{0} u=f$. The change of variables $u(t)=e^{\left(\lambda_{1}-\delta\right) t} v(t)$ transforms it into

$v^{\prime \prime}(t)-\left(c_{0}-2\left(\lambda_{1}-\delta\right)\right) v^{\prime}(t)+\left(\left(\lambda_{1}-\delta\right)^{2}-c_{0}\left(\lambda_{1}-\delta\right)+a(t)\right) v(t)+e^{-\left(\lambda_{1}-\delta\right) h_{0}} b(t) v\left(t-h_{0}\right)=f_{1}(t)$.

By our assumptions on $f$ and $\delta$, the function $f_{1}(t)$ is bounded and the limit equation for the latter equation at $-\infty$,

$v^{\prime \prime}(t)-\left(c_{0}-2\left(\lambda_{1}-\delta\right)\right) v^{\prime}(t)+\left(\left(\lambda_{1}-\delta\right)^{2}-c_{0}\left(\lambda_{1}-\delta\right)+a_{-}\right) v(t)+e^{-\left(\lambda_{1}-\delta\right) h_{0}} b_{-} v\left(t-h_{0}\right)=0$,

is exponentially dichotomic on $\mathbb{R}$. Then the well known results from the exponential dichotomy theory (e.g., see Lemmas 3.2 and 4.3 in [15]) show that the homogeneous equation

$v^{\prime \prime}(t)-\left(c_{0}-2\left(\lambda_{1}-\delta\right)\right) v^{\prime}(t)+\left(\left(\lambda_{1}-\delta\right)^{2}-c_{0}\left(\lambda_{1}-\delta\right)+a(t)\right) v(t)+e^{-\left(\lambda_{1}-\delta\right) h_{0}} b(t) v\left(t-h_{0}\right)=0$

possesses an exponential dichotomy on $(-\infty, T]$ so that the above considered inhomogeneous equation has at least one bounded solution $v_{*}(t), t \leq T$, with $v_{*}^{\prime}(t), v_{*}^{\prime \prime}(t)$ which are also bounded on $\mathbb{R}_{-}$. It is clear that $u_{*}(t)=e^{\left(\lambda_{1}-\delta\right) t} v_{*}(t) \in \tilde{X}_{\delta}$ and $\mathcal{D}_{0} u_{*}=f$.

The smoothness properties of operator $N$ and the Fredholm property of $\mathcal{D}_{0}$ allow to realize a standard Lyapunov-Schmidt reduction in the equation $\mathcal{D}_{0} \zeta(t)=N(\zeta, c, h)(t)$. The details of this procedure (used in more complex situation) are described in Lemma 5.7 below. This method allows to establish the existence of one-parametric family of functions $\zeta=\zeta(t, h, c, a)$ depending $C^{1}$-smoothly on parameters $(h, c, a)$ close to $\left(h_{0}, c_{0}, 0\right)$ and such that $\zeta\left(t, h_{0}, c_{0}, 0\right)=0$ for all $t \leq T$ and $\phi_{0}(t)+\zeta(t, c, h, a)$ solves equation (31) for each fixed $(c, h, a)$. Note that the dimension 1 of parameter $a$ corresponds to $\operatorname{dim} \operatorname{Ker} \mathcal{D}_{0}=1$. We can reintroduce this parameter in a more usual way by fixing $a=0$ and considering the family of shifted solutions $\phi_{0}(t+s)+\zeta(t+s, c, h, 0)$ of equation (31).

Finally, consider the equation $\phi_{0}(T+s)+\zeta(T+s, c, h, 0)=\phi_{0}(T)$. Clearly $(s, h, c)=\left(0, h_{0}, c_{0}\right)$ is a solution of this equation while $\phi_{0}^{\prime}(T)>0$. Therefore, in view of the implicit function theorem, there exists $C^{1}$-smooth solution $s=s(c, h)$ of this equation satisfying equality $s\left(h_{0}, c_{0}\right)=0$. We obtain the required family $\psi_{*}$ by setting

$$
\psi_{*}(t, c, h)=\phi_{0}(t+s(c, h))+\zeta(t+s(c, h), c, h, 0) .
$$

Observe also that the monotonicity properties of $\psi_{*}(t, c, h)$ are assured by Lemma 3.7 and

$$
\frac{\partial \psi_{*}(\cdot, c, h)}{\partial c}=\left(\phi_{0}^{\prime}(\cdot+s(c, h))+\zeta^{\prime}(\cdot+s(c, h), c, h, 0)\right) s_{1}(c, h)+\zeta_{2}(\cdot+s(c, h), c, h, 0) \in \tilde{X}_{\delta}
$$

Next, using $C^{\infty}$-smooth non-increasing function $S_{\varepsilon}(t)$ such that $S_{\varepsilon}(t)=1$ for $t \leq T$ and $S_{\varepsilon}(t)=0$ for $t \geq T+\varepsilon$, we will define the 'corrector' $\psi_{\varepsilon}(t, c, h)=$ $\left(\psi_{*}(t, c, h)-\phi_{0}(t)\right) S_{\varepsilon}(t)$. Clearly, $\partial \psi_{*}(T, c, h) / \partial c=0$ and

$$
\psi_{\varepsilon}(t, c, h)=\left\{\begin{array}{cc}
\psi_{*}(t, c, h)-\phi_{0}(t), & t \leq T, \\
0, & t \geq T+\varepsilon .
\end{array}\right.
$$


Also $\psi_{\varepsilon}\left(t, c_{0}, h_{0}\right) \equiv 0$. We will look for a monotone solution $\phi(t, c, h), t \in \mathbb{R}$, of (31) in the form

$$
\phi(t, c, h)=\phi_{0}(t)+\psi_{\varepsilon}(t, c, h)+\zeta(t, c, h),
$$

where $\zeta \in X_{\delta}$. Then the equation for $\zeta$ is $\mathcal{D}_{0} \zeta(t)=N_{\varepsilon}(\zeta, c, h)(t)$, where $\mathcal{D}_{0}$ is given by (36) and $N_{\varepsilon}(\zeta, c, h)(t)=$

$$
\left(c-c_{0}\right)\left(\phi_{0}^{\prime}(t)+\zeta^{\prime}(t)\right)+c\left(\left(\psi_{*}(t)-\phi_{0}(t)\right) S_{\varepsilon}(t)\right)^{\prime}-\left(\left(\psi_{*}(t)-\phi_{0}(t)\right) S_{\varepsilon}(t)\right)^{\prime \prime}+a(t) \zeta(t)+
$$

$b(t) \zeta\left(t-h_{0}\right)+g\left(\phi_{0}(t), \phi_{0}\left(t-h_{0}\right)\right)-g\left(\phi_{0}(t)+\psi_{\varepsilon}(t)+\zeta(t), \phi_{0}(t-h)+\psi_{\varepsilon}(t-h)+\zeta(t-h)\right), t \in \mathbb{R}$.

Since $\psi_{*}(t, c, h)=\phi_{0}(t)+\psi_{\varepsilon}(t, c, h), t \leq T$, solves equation (31) for all $t \leq T$, it is easy to find that

$$
\begin{gathered}
N_{\varepsilon}(\zeta, c, h)(t)=\left(c-c_{0}\right) \zeta^{\prime}(t)+a(t) \zeta(t)+b(t) \zeta\left(t-h_{0}\right)+ \\
+g\left(\psi_{*}(t, c, h), \psi_{*}(t-h, c, h)\right)-g\left(\psi_{*}(t, c, h)+\zeta(t), \psi_{*}(t-h, c, h)+\zeta(t-h)\right), \quad t \leq T .
\end{gathered}
$$

$N_{\varepsilon}$ has the following smoothness properties:

Lemma 5.5 There exist neighborhoods $\mathcal{O}\left(c_{0}\right)$ and $\mathcal{O}\left(h_{0}\right)$ of the points $c_{0}, h_{0}$ such that function $N_{\varepsilon}: X_{\delta} \times \mathcal{O}\left(c_{0}\right) \times \mathcal{O}\left(h_{0}\right) \rightarrow Y_{\delta}, N_{\varepsilon}\left(0, c_{0}, h_{0}\right)=0$, is continuously differentiable, with continuous partial derivatives given by

$$
\begin{gathered}
D_{c} N_{\varepsilon}(\zeta, c, h)(t)=\phi_{0}^{\prime}(t)+\zeta^{\prime}(t)-\left(\phi_{0}(t) S_{\varepsilon}(t)\right)^{\prime}+S_{\varepsilon}(t) D_{c} g\left(\psi_{*}(t), \psi_{*}(t-h)\right)-\left(\psi_{*}\right)_{c}(t) S_{\varepsilon}^{\prime \prime}(t)+ \\
\left(\psi_{*}(t)+c\left(\psi_{*}\right)_{c}(t)-2\left(\psi_{*}^{\prime}(t)\right)_{c}\right) S_{\varepsilon}^{\prime}(t)-D_{c} g\left(\phi_{0}(t)+\psi_{\varepsilon}(t)+\zeta(t), \phi_{0}(t-h)+\psi_{\varepsilon}(t-h)+\zeta(t-h)\right), t \in \mathbb{R} ; \\
D_{c} N_{\varepsilon}(\zeta, c, h)(t)=\zeta^{\prime}(t)+D_{c} g\left(\psi_{*}(t, c, h), \psi_{*}(t-h, c, h)\right)- \\
D_{c} g\left(\psi_{*}(t, c, h)+\zeta(t), \psi_{*}(t-h, c, h)+\zeta(t-h)\right), t \leq T . \\
D_{h} N_{\varepsilon}(\zeta, c, h)=S_{\varepsilon}(t) D_{h} g\left(\psi_{*}(t, c, h), \psi_{*}(t-h, c, h)\right)+S_{\varepsilon}^{\prime}(t)\left(c\left(\psi_{*}\right)_{h}(t)-2\left(\psi_{*}^{\prime}\right)_{h}(t)\right)- \\
-\left(\psi_{*}\right)_{h}(t) S_{\varepsilon}^{\prime \prime}(t)-D_{h} g(\phi(t, c, h), \phi(t-h, c, h)) \\
D_{\zeta} N_{\varepsilon}(\zeta, c, h) w(t)=\left(c-c_{0}\right) w^{\prime}(t)+a(t) w(t)+b(t) w\left(t-h_{0}\right)- \\
g_{1}\left(\phi_{0}(t)+\psi_{\varepsilon}(t)+\zeta(t), \phi_{0}(t-h)+\psi_{\varepsilon}(t-h)+\zeta(t-h)\right) w(t)- \\
g_{2}\left(\phi_{0}(t)+\psi_{\varepsilon}(t)+\zeta(t), \phi_{0}(t-h)+\psi_{\varepsilon}(t-h)+\zeta(t-h)\right) w(t-h) .
\end{gathered}
$$

In particular, $D_{\zeta} N_{\varepsilon}\left(0, c_{0}, h_{0}\right)=0$,

$$
D_{c} N_{\varepsilon}\left(0, c_{0}, h_{0}\right)(t)=\left\{\begin{array}{cc}
0, & t \leq T, \\
\phi_{0}^{\prime}(t)+R_{\varepsilon}(t), & t \geq T, \\
\phi_{0}^{\prime}(t), & t \geq T+\varepsilon,
\end{array}\right.
$$

where $R_{\varepsilon}(t)=S_{\varepsilon}(t) D_{c} g\left(\psi_{*}(t), \psi_{*}\left(t-h_{0}\right)\right)+\left(c_{0}\left(\psi_{*}\right)_{c}(t)-2\left(\psi_{*}^{\prime}(t)\right)_{c}\right) S_{\varepsilon}^{\prime}(t)-\left(\psi_{*}\right)_{c}(t) S_{\varepsilon}^{\prime \prime}(t)+$ $-D_{c} g\left(\phi_{0}(t)+\psi_{\varepsilon}(t), \phi_{0}\left(t-h_{0}\right)+\psi_{\varepsilon}\left(t-h_{0}\right)\right)-\phi_{0}^{\prime}(t) S_{\varepsilon}(t)$. 
Proof. The proof of this lemma is based on routine straightforward calculations. Some of them (concerning $D_{\zeta} N_{\varepsilon}$ ) are given below, in the proof of a similar technical assertion, Lemma 5.8. Here it is convenient to use the relation $\mathcal{T}:=c\left(\left(\psi_{*}(t)-\phi_{0}(t)\right) S_{\varepsilon}(t)\right)^{\prime}-$ $\left(\left(\psi_{*}(t)-\phi_{0}(t)\right) S_{\varepsilon}(t)\right)^{\prime \prime}=-c\left(\phi_{0}(t) S_{\varepsilon}(t)\right)^{\prime}+$

$$
\left(\phi_{0}(t) S_{\varepsilon}(t)\right)^{\prime \prime}+S_{\varepsilon}(t) g\left(\psi_{*}(t, c, h), \psi_{*}(t-h, c, h)\right)+\left(c \psi_{*}(t)-2 \psi_{*}^{\prime}(t)\right) S_{\varepsilon}^{\prime}(t)-\psi_{*}(t) S_{\varepsilon}^{\prime \prime}(t) .
$$

Recall that $\psi_{*}(t, \cdot, h): \mathcal{O}\left(c_{0}\right) \rightarrow \tilde{X}_{\delta}$ depends $C^{1}$-continuously on $c \in \mathcal{O}\left(c_{0}\right)$ while all terms of $\mathcal{T}$ belong to the space $Y_{\delta}$ since $S_{\varepsilon}(t)=0$ for all $t \geq T+\varepsilon$.

Corollary 5.6 It holds that

$$
\lim _{\varepsilon \rightarrow 0^{+}} \int_{-\infty}^{+\infty} w_{*}(t) D_{c} N_{\varepsilon}\left(0, c_{0}, h_{0}\right)(t) d t=\lim _{\varepsilon \rightarrow 0^{+}} \int_{T}^{T+\varepsilon} w_{*}(t) R_{\varepsilon}(t) d t+\int_{T}^{+\infty} w_{*}(t) \phi_{0}^{\prime}(t) d t=
$$

$=\int_{T}^{+\infty} w_{*}(t) \phi_{0}^{\prime}(t) d t>0$, so that $\int_{-\infty}^{+\infty} w_{*}(t) D_{c} N_{\varepsilon}\left(0, c_{0}, h_{0}\right)(t) d t>0$ for all small positive $\varepsilon$.

Proof. Indeed, by integrating by parts and using the boundary conditions $w_{*}(T)=$ $\left(\psi_{*}\right)_{c}(T)=0$, we find that $\int_{T}^{T+\varepsilon} w_{*}(t) R_{\varepsilon}(t) d t=$

$$
\begin{aligned}
& \int_{T}^{T+\varepsilon} w_{*}(t)\left\{S_{\varepsilon}(t) D_{c} g\left(\psi_{*}(t), \psi_{*}\left(t-h_{0}\right)\right)-D_{c} g\left(\phi_{0}(t)+\psi_{\varepsilon}(t), \phi_{0}\left(t-h_{0}\right)+\psi_{\varepsilon}\left(t-h_{0}\right)\right)\right\} d t \\
& -\int_{T}^{T+\varepsilon} S_{\varepsilon}(t)\left(\phi_{0}^{\prime}(t) w_{*}(t)+\left[w_{*}(t)\left(c_{0}\left(\psi_{*}\right)_{c}(t)-2\left(\psi_{*}^{\prime}(t)\right)_{c}\right)\right]^{\prime}+\left(w_{*}(t)\left(\psi_{*}\right)_{c}(t)\right)^{\prime \prime}\right) d t=O(\epsilon)
\end{aligned}
$$

Lemma 5.7 Suppose that $\phi_{0}^{\prime}(t)>0, c_{0}>0$, and that hypothesis (U) is satisfied. Then there exist an open neighbourhood $\mathcal{O}$ of $h_{0}=\tau_{0} c_{0}$, and $C^{1}$-smooth function $c: \mathcal{O} \rightarrow(0,+\infty), c\left(h_{0}\right)=c_{0}$, such that equation (31) has a continuous family $\phi(\cdot, c(h), h) \in \phi_{0}+X_{\delta}, \phi\left(t, c\left(h_{0}\right), h_{0}\right)=\phi_{0}(t)$, of strictly increasing bistable wavefronts.

Proof. Consider the direct sums of Banach spaces $X_{\delta}=\operatorname{Ker} \mathcal{D}_{0} \oplus W, Y_{\delta}=R\left(\mathcal{D}_{0}\right) \oplus V$, where $\operatorname{Ker} \mathcal{D}_{0}$ is one-dimensional null space of the linear operator $\mathcal{D}_{0}$ and the range $R\left(\mathcal{D}_{0}\right)$ has codimension one,

$$
\begin{gathered}
R\left(\mathcal{D}_{0}\right)=\left\{f \in Y_{\delta}: \int_{\mathbb{R}} f(s) w_{*}(s) d s=0\right\}, \\
V=<y_{*}>\text { for some } y_{*} \in Y_{\delta} \text { with } \int_{\mathbb{R}} w_{*}(u) y_{*}(u) d u \neq 0 .
\end{gathered}
$$

Let $P: Y_{\delta} \rightarrow Y_{\delta}$ be the projection on the subspace $R\left(\mathcal{D}_{0}\right)$ along $V$,

$$
P f(s)=f(s)-\frac{\int_{\mathbb{R}} f(u) w_{*}(u) d u}{\int_{\mathbb{R}} w_{*}(u) y_{*}(u) d u} y_{*}(s) .
$$

Then the equation $\mathcal{D}_{0} \zeta=N_{\varepsilon}(\zeta, c, h), \zeta \in X_{\delta}$, is equivalent to the system

$$
\mathcal{D}_{0} \xi=P N_{\varepsilon}(\xi+u, c, h), \xi \in W \subset X_{\delta}, u=k \phi_{0}^{\prime}, \quad(I-P) N_{\varepsilon}(\xi+u, c, h)=0
$$


in the sense that $\zeta=\xi+u$ satisfies the former equation if and only if it satisfies the latter system. Considering the restriction $\mathcal{D}^{\prime}=\left.\mathcal{D}_{0}\right|_{W}: W \rightarrow R(\mathcal{D})$, we know that the operator $\mathcal{D}^{\prime}$ is invertible and thus the equation $\mathcal{D}^{\prime} \xi(t)=P N_{\varepsilon}(\xi+u, c, h)$ can be written as $\xi=\left(\mathcal{D}^{\prime}\right)^{-1} P N_{\varepsilon}(\xi+u, c, h)=Q(\xi+u, c, h)$. Since $D_{\xi} Q\left(0, c_{0}, h_{0}\right)=0$, the implicit function theorem [2] shows that this equation has a $C^{1}$-continuous family of solutions $\xi=\xi(u, c, h)$ defined in some vicinity of the point $\left(0, c_{0}, h_{0}\right)$, where $\xi\left(0, c_{0}, h_{0}\right)=0$. We still need to prove that for appropriate parameters $(c, h)$ close to $\left(c_{0}, h_{0}\right)$ the equation

$$
(I-P) N_{\varepsilon}(\xi(u, c, h)+u, c, h)=0
$$

is satisfied. Simplifying, we can take $u=0$. Since for all small $\varepsilon>0$, in view of Corollary 5.6,

$$
\begin{gathered}
\left.D_{c}(I-P) N_{\varepsilon}\left(\xi\left(0, c, h_{0}\right), c, h_{0}\right)\right|_{c=c_{0}}=(I-P) D_{c} N_{\varepsilon}\left(0, c_{0}, h_{0}\right)= \\
\frac{\int_{\mathbb{R}} D_{c} N_{\varepsilon}\left(0, c_{0}, h_{0}\right)(u) w_{*}(u) d u}{\int_{\mathbb{R}} w_{*}(u) y_{*}(u) d u} y_{*}(s) \neq 0,
\end{gathered}
$$

we conclude that there exists a $C^{1}$-continuous solution $c=c(h), c\left(h_{0}\right)=c_{0}, h \in \mathcal{O}$, of the equation $(I-P) N_{\varepsilon}(\xi(0, c, h), c, h)=0$. To finalise the proof of the lemma, we have to establish the monotonicity of bistable waves

$$
\phi(t, c(h), h):=\phi_{0}(t)+\psi_{\varepsilon}(t, c, h)+\xi(0, c(h), h)(t), h \in \mathcal{O} .
$$

First, note that Lemmas 3.3, 3.7 imply that $\phi(\cdot, c(h), h): \mathbb{R} \rightarrow\left(e_{1}, e_{3}\right)$ for all $h \in \mathcal{O}$. Moreover, each $\phi(t, c(h), h)$ is strictly monotone in $t$ on some maximal interval $\left(-\infty, s_{h}\right)$, where $\phi\left(s_{h}, c(h), h\right)>\kappa$, and is also strictly monotone at $+\infty$, see Lemma 3.4. In fact, we prove below that the following asymptotic formula holds at $+\infty$ :

$\phi^{\prime}(t, c(h), h)=q_{1}(h) e^{\mu_{2} t}+r(t, h), \quad$ where $|r(t, h)| \leq K e^{\left(\mu_{2}-\delta^{\prime}\right) t}, t \geq 0, h \in \mathcal{O}^{\prime} \subset \mathcal{O},(37)$ where $K \geq 1, \delta^{\prime}>0$ does not depend on $h$, and $q_{1}(h)$ is a positive continuous function defined on some smaller neighbourhood $\mathcal{O}^{\prime}$ of $h_{0}$. It follows from (37) that $\phi^{\prime}(t, c(h), h)>0$ for all $h \in \mathcal{O}^{\prime}$ and $t>t_{*}:=\left(\delta^{\prime}\right)^{-1} \sup \left\{\ln \left(K / q_{1}(h)\right), h \in \mathcal{O}^{\prime}\right\}$. Since $\phi^{\prime}(t, c(h), h)$ converges to $\phi_{0}^{\prime}(t)>0$ (as $h \rightarrow h_{0}$ ) uniformly on compact subsets of $\mathbb{R}$, we may conclude that $\phi^{\prime}(t, c(h), h)>0$ for all $t \in \mathbb{R}$ once $h$ is sufficiently close to $h_{0}$.

To prove (37), we apply the bilateral Laplace transform to the differential equation for $\psi(t):=e_{3}-\phi(t, c(h), h)$ :

$$
\psi^{\prime \prime}(t)-c \psi^{\prime}(t)+a_{+} \psi(t)+b_{+} \psi(t-h)=d(t, h),
$$

where $d(t, h)=-\left(g\left(e_{3}, e_{3}\right)-g\left(e_{3}-\psi(t), e_{3}-\psi(t-h)\right)-a_{+} \psi(t)-b_{+} \psi(t-h)\right)=$ $O\left(e^{\left(\mu_{2}\left(h_{0}\right)+\delta\right)(1+\gamma) t}\right), t \rightarrow+\infty$. Importantly, since the function $\xi(0, c(h), h): \mathcal{O} \rightarrow X_{\delta}$ is continuous, the latter $O$ relation for $d(t, h)$ is satisfied uniformly with respect to $h$ from compact subsets of $\mathcal{O}$. Thus, for some small positive $r>0$ satisfying $\mu_{2}(h)-r>\left(\mu_{2}\left(h_{0}\right)+\delta\right)(1+\gamma)$ and $t>0$, we have that

$$
\psi(t)=\operatorname{Res}_{z=\mu_{2}} \frac{e^{z t} \tilde{d}(z, h)}{\chi_{+}(z)}+\frac{1}{2 \pi i} \int_{\Re z=\mu_{2}-r} \frac{e^{t z} \tilde{d}(z, h)}{\chi_{+}(z)} d z=\alpha(h) e^{\mu_{2} t}+B(t, h),
$$


with $B(t, h):=\beta(t, h) e^{\left(\mu_{2}-r\right) t}$. Here $\tilde{d}(z, h)=\int_{\mathbb{R}} e^{-z s} d(s, h) d s, \Re z \in\left((1+\gamma)\left(\mu_{2}\left(h_{0}\right)+\right.\right.$ $\delta), 0)$, is the bilateral Laplace transform of $d(t, h)$. In view of the Lebesgue dominated convergence theorem and the uniform (with respect to $h$ from compact subsets of $\mathcal{O})$ exponential estimate $d(t, h)=O\left(e^{\left(\mu_{2}\left(h_{0}\right)+\delta\right)(1+\gamma) t}\right), t \rightarrow+\infty$, the transform $\tilde{d}(z, h)$ depends continuously on $z, h$ and is uniformly bounded on the vertical line $\left\{\Re z=\mu_{2}-r\right\}$.

Consequently, in view of Lemma 3.4, there exists some small neighbourhood $\mathcal{O}^{\prime} \subset \mathcal{O}$ of $h_{0}$ such that continuous functions $\alpha(t), \beta(t, h)$ satisfy the estimates

$$
\alpha(h)=\frac{\tilde{d}\left(\mu_{2}(h), h\right)}{\chi_{+}^{\prime}\left(\mu_{2}(h)\right)}>0, \quad|\beta(t, h)| \leq \frac{1}{2 \pi} \int_{\mathbb{R}} \frac{\left|\tilde{d}\left(\mu_{2}(h)-r+i s, h\right)\right|}{\left|\chi_{+}\left(\mu_{2}(h)-r+i s\right)\right|} d s \leq B_{0}, h \in \mathcal{O}^{\prime}
$$

where $B_{0}=B_{0}\left(\mathcal{O}^{\prime}\right)$ is some positive constant. Finally, integrating $(38)$ on $(t,+\infty)$, we find that

$$
\psi^{\prime}(t)=c \psi(t)+\int_{t}^{+\infty}\left(a_{+} \psi(s)+b_{+} \psi(s-h)-d(s, h)\right) d s=\mu_{2}(h) \alpha(h) e^{\mu_{2}(h) t}+R(t, h),
$$

where

$$
R(t, h)=c B(t, h)+\int_{t}^{+\infty}\left(a_{+} B(s, h)+b_{+} B(s-h, h)-d(s, h)\right) d s
$$

satisfies $|R(t, h)| \leq D e^{\left(\mu_{2}-r\right) t}, t>0, h \in \mathcal{O}^{\prime}$, with $D$ not depending on $h$.

\subsection{Local continuation of wavefronts under hypothesis ( $\left.\mathbf{U}^{*}\right)$.}

When $\left(\mathbf{U}^{*}\right)$ is assumed instead of $(\mathbf{U})$, the local continuation of wavefronts is somewhat easier to prove. The main reason of this is the non-negativity of solution $w_{*}(t)$ of the adjoint equation. Indeed, at the beginning of Subsection 5.2, we mentioned that the proofs in this subsection simplify when $w_{*}(t) \geq 0, t \in \mathbb{R}$ (i.e. when $T=-\infty$ ). Therefore in Subsection 5.2 we narrowed our attention to more complex case of finite $T$. In the present subsection we show how the Lyapunov-Schmidt reduction works for $T=-\infty$.

Hence, suppose that, given $\tau_{0} \geq 0$, equation (1) has a monotone bistable wavefront $u(t, x)=\phi_{0}\left(t+c_{0} t\right), c_{0}>0$. For $c, h$ close to $c_{0}, h_{0}=c_{0} \tau_{0}$, we will look for a monotone solution $\phi(t, c, h)$ of (31) in the form

$$
\phi(t, c, h)=\phi_{0}(t)+\zeta(t, c, h)
$$

where $\zeta \in X_{\delta}$. Then the equation for $\zeta$ is $\mathcal{D}_{0} \zeta(t)=N(\zeta, c, h)$, where $\mathcal{D}_{0} \zeta, N(\zeta, c, h)$ are defined in (36). Next result can be regarded as somewhat simplified version of Lemma 5.5:

Lemma 5.8 Function $N: X_{\delta} \times(0,+\infty) \times[0,+\infty) \rightarrow Y_{\delta}$ is continuously differentiable, with continuous partial derivatives given by $D_{c} N(\zeta, c, h)=\phi_{0}^{\prime}(t)+\zeta^{\prime}(t)$,

$$
\begin{gathered}
D_{h} N(\zeta, c, h)=g_{2}\left(\phi_{0}(t)+\zeta(t), \phi_{0}(t-h)+\zeta(t-h)\right)\left(\phi_{0}^{\prime}(t-h)+\zeta^{\prime}(t-h)\right) \\
D_{\zeta} N(\zeta, c, h) w(t)=\left(c-c_{0}\right) w^{\prime}(t)+a(t) w(t)+b(t) w\left(t-h_{0}\right)
\end{gathered}
$$


$-g_{1}\left(\phi_{0}(t)+\zeta(t), \phi_{0}(t-h)+\zeta(t-h)\right) w(t)-g_{2}\left(\phi_{0}(t)+\zeta(t), \phi_{0}(t-h)+\zeta(t-h)\right) w(t-h)$.

In particular, $N\left(0, c_{0}, h_{0}\right)=0, \quad D_{\zeta} N\left(0, c_{0}, h_{0}\right)=0, \quad D_{c} N\left(0, c_{0}, h_{0}\right)=\phi_{0}^{\prime}(t)$,

$$
D_{h} N\left(0, c_{0}, h_{0}\right)=g_{2}\left(\phi_{0}(t), \phi_{0}\left(t-h_{0}\right)\right) \phi_{0}^{\prime}\left(t-h_{0}\right) \text {. }
$$

Proof. Clearly, it suffices to check the validity of the conclusions of Lemma 5.8 only for the nonlinear part $N_{1}$ of $N$. Here $N_{1}(\zeta, h)=g\left(\phi_{0}(t), \phi_{0}\left(t-h_{0}\right)\right)-g\left(\phi_{0}(t)+\zeta(t), \phi_{0}(t-\right.$ $h)+\zeta(t-h))$, and below we will give details of computations only for more difficult derivative $D_{\zeta} N_{1}$, the other derivatives being similar. To abbreviate, we use the notation $f_{h}(t)=f(t-h)$. First, we find that $\Delta:=$

$N_{1}(\zeta+w, h)-N_{1}(\zeta, h)+g_{1}\left(\phi_{0}(t)+\zeta(t), \phi_{h}(t)+\zeta_{h}(t)\right) w(t)+g_{2}\left(\phi_{0}(t)+\zeta(t), \phi_{h}(t)+\zeta_{h}(t)\right) w_{h}(t)=$

$\int_{0}^{1}\left(g_{1}\left(\phi_{0}(t)+\zeta(t), \phi_{h}(t)+\zeta_{h}(t)\right)-g_{1}\left(\phi_{0}(t)+\zeta(t)+s w(t), \phi_{h}(t)+\zeta_{h}(t)+s w_{h}(t)\right) d s w(t)+\right.$ $\int_{0}^{1}\left(g_{2}\left(\phi_{0}(t)+\zeta(t), \phi_{h}(t)+\zeta_{h}(t)\right)-g_{2}\left(\phi_{0}(t)+\zeta(t)+s w(t), \phi_{h}(t)+\zeta_{h}(t)+s w_{h}(t)\right) d s w_{h}(t)\right.$.

Therefore, for every $r>0$ there exists $K_{r}$ such that for all $w$ such that $|w|_{\infty} \leq r$, it holds

$$
|\Delta| \leq K_{r}\left(|w(t)|^{1+\gamma}+|w(t-h)|^{\gamma}|w(t)|+|w(t)|^{\gamma}|w(t-h)|+|w(t-h)|^{1+\gamma}\right) .
$$

The latter implies that $|\Delta|_{Y_{\delta}} \leq K_{r}^{\prime}\left(|w|_{Y_{\delta}}^{1+\gamma}\right)$ for some $K_{r}^{\prime} \geq K_{r}$ and all $w$ such that $|w|_{Y_{\delta}} \leq r$. This proves that the Fréchet derivative $D_{\zeta} N_{1}$ exists and is given by $D_{\zeta} N_{1}(\zeta, h) w(t)=$

$-g_{1}\left(\phi_{0}(t)+\zeta(t), \phi_{0}(t-h)+\zeta(t-h)\right) w(t)-g_{2}\left(\phi_{0}(t)+\zeta(t), \phi_{0}(t-h)+\zeta(t-h)\right) w(t-h)$.

Next, it can proved similarly that $D_{\zeta} N_{1}(\zeta, h)$ is locally Hölder continuous function in view of the estimate

$\left\|D_{\zeta} N_{1}\left(\zeta_{1}, h_{1}\right)-D_{\zeta} N_{1}(\zeta, h)\right\| \leq K\left(\left|\zeta_{1}-\zeta\right|_{\infty}^{\gamma}+\left[\left|\zeta^{\prime}\right|_{\infty}+\left|\phi_{0}^{\prime}\right|_{\infty}\right]^{\gamma}\left|h-h_{1}\right|^{\gamma}+\left|h-h_{1}\right|\right)$.

Lemma 5.9 Suppose that $\phi_{0}^{\prime}(t)>0, c_{0}>0$ and that hypothesis $\left(\mathbf{U}^{*}\right)$ with $c_{0}<$ $\operatorname{clin}\left(h_{0} / c_{0}\right)$ is satisfied. Then there exist an open neighbourhood $\mathcal{O}$ of $h_{0}=\tau_{0} c_{0}$, and $C^{1}$ smooth function $c: \mathcal{O} \rightarrow(0,+\infty), c\left(h_{0}\right)=c_{0}$, such that equation (31) has a continuous family $\phi(\cdot, c(h), h) \in \phi_{0}+X_{\delta}, h \in \mathcal{O}, \phi\left(t, c\left(h_{0}\right), h_{0}\right)=\phi_{0}(t)$, of strictly increasing bistable wavefronts. If $\left(\mathbf{U}^{*}\right)$ holds with $c_{0}=c l i n\left(h_{0} / c_{0}\right)$, the same conclusion, possibly except for the strict monotonicity property of $\phi(\cdot, c(h), h)$ at $+\infty$, is true.

Proof. Taking the non-negative solution $w_{*}(t)$ defined in Section 4, we consider the Banach spaces $W, V$ and the projector $P: Y_{\delta} \rightarrow Y_{\delta}$ defined in the first paragraphs of 
the proof of Lemma 5.7. Then the equation $\mathcal{D}_{0} \zeta(t)=N(\zeta, c, h), \zeta \in X_{\delta}$, is equivalent to the system

$$
\mathcal{D}_{0} \xi(t)=P N(\xi+u, c, h), \xi \in W \subset X_{\delta}, u=k \phi_{0}^{\prime}, \quad(I-P) N(\xi+u, c, h)=0 .
$$

Considering the restriction $\mathcal{D}^{\prime}=\left.\mathcal{D}_{0}\right|_{W}: W \rightarrow R(\mathcal{D})$, we know that the operator $\mathcal{D}^{\prime}$ is invertible and thus the equation $\mathcal{D}^{\prime} \xi(t)=P N(\xi+u, c, h)$ can be written as $\xi=\left(\mathcal{D}^{\prime}\right)^{-1} P N(\xi+u, c, h)=Q(\xi+u, c, h)$. Since $D_{\xi} Q\left(0, c_{0}, h_{0}\right)=0$, this equation has a $C^{1}$-continuous family of solutions $\xi=\xi(u, c, h)$ defined in some vicinity of the point $\left(0, c_{0}, h_{0}\right)$, where $\xi\left(0, c_{0}, h_{0}\right)=0$. We have to prove that for appropriate parameters $(c, h)$ close to $\left(c_{0}, h_{0}\right)$ the equation

$$
(I-P) N(\xi(u, c, h)+u, c, h)=0
$$

is satisfied. It suffices to take $u=0$. Since

$\left.D_{c}(I-P) N\left(\xi\left(0, c, h_{0}\right), c, h_{0}\right)\right|_{c=c_{0}}=(I-P) D_{c} N\left(0, c_{0}, h_{0}\right)=\frac{\int_{\mathbb{R}} \phi_{0}^{\prime}(u) w_{*}(u) d u}{\int_{\mathbb{R}} w_{*}(u) y_{*}(u) d u} y_{*}(s) \neq 0$, we conclude that there exists a $C^{1}$-continuous solution $c=c(h), c\left(h_{0}\right)=c_{0}, h \in \mathcal{O}$, of the equation $(I-P) N(\xi(0, c, h), c, h)=0$.

For $c_{0}>c^{\mathfrak{E}}\left(h_{0}\right)$, we will prove now the monotonicity of the obtained bistable waves

$$
\phi(t, c(h), h):=\phi_{0}(t)+\xi(0, c(h), h)(t), h \in \mathcal{O} .
$$

The restriction $c_{0}>c^{\mathfrak{E}}\left(h_{0}\right)$ implies that $\chi_{-}(z)$ has exactly three different real zeros, $\lambda_{3}<\lambda_{2}<0<\lambda_{1}$. By Lemma 4.4, $\phi(t, c(h), h)$ is strictly monotone in $t$ on some maximal interval $\left(-\infty, r_{h}\right)$, where $\phi\left(r_{h}, c(h), h\right) \geq e_{2}$. Therefore, to complete the proof of Lemma 5.7, it suffices to prove the following asymptotic formula (which is similar to $(37))$ :

$\phi^{\prime}(t, c(h), h)=q_{2}(h) e^{\lambda_{2} t}+r_{2}(t, h)$, where $\left|r_{2}(t, h)\right| \leq K_{2} e^{\left(\lambda_{2}-\delta^{\prime \prime}\right) t}, t \geq 0, h \in \mathcal{O}^{\prime \prime} \subset \mathcal{O},(39)$ where $K_{2} \geq 1, \delta^{\prime \prime}>0$ does not depend on $h$, and $q_{2}(h)$ is a positive continuous function defined on some small neighbourhood $\mathcal{O}^{\prime \prime}$ of $h_{0}$. Indeed, once (39) is established, we can argue as in the paragraph below formula (37).

Now, in order to prove (39), we will apply the bilateral Laplace transform to the differential equation for $\psi(t):=e_{3}-\phi(t, c(h), h)$ :

$$
\psi^{\prime \prime}(t)-c \psi^{\prime}(t)+a_{-} \psi(t)+b_{-} \psi(t-h)=d_{*}(t, h),
$$

where $d_{*}(t, h)=-\left(g\left(e_{3}, e_{3}\right)-g\left(e_{3}-\psi(t), e_{3}-\psi(t-h)\right)-a_{-} \psi(t)-b_{-} \psi(t-h)\right), t \in \mathbb{R}$. Clearly, in view of the sub-tangency restriction imposed in $\left(\mathbf{U}^{*}\right)$,

$d_{*}\left(t, h_{0}\right)=-\left(g\left(e_{3}, e_{3}\right)-g\left(e_{3}-\phi_{0}(t), e_{3}-\phi_{0}(t-h)\right)-a_{-} \phi_{0}(t)-b_{-} \phi_{0}\left(t-h_{0}\right)\right) \leq 0, t \in \mathbb{R}$.

Also, $d_{*}\left(t, h_{0}\right) \not \equiv 0$ on $\mathbb{R}$ and $d_{*}(t, h)=O\left(e^{\left(\lambda_{2}\left(h_{0}\right)+\delta\right)(1+\gamma) t}\right), t \rightarrow+\infty$, with $O$ relation being satisfied uniformly with respect to $h \in \mathcal{O}^{\prime \prime}$. Thus, for some small positive $r^{\prime}>0$ satisfying $\lambda_{2}(h)-r^{\prime}>\left(\lambda_{2}\left(h_{0}\right)+\delta\right)(1+\gamma)$ and $t>0$, we have that $\tilde{d}_{*}\left(\lambda_{2}\left(h_{0}\right), h_{0}\right)>0$,

$$
\psi(t)=\operatorname{Res}_{z=\lambda_{2}} \frac{e^{z t} \tilde{d}_{*}(z, h)}{\chi_{-}(z)}+\frac{1}{2 \pi i} \int_{\Re z=\lambda_{2}-r^{\prime}} \frac{e^{t z} \tilde{d}_{*}(z, h)}{\chi_{-}(z)} d z=\alpha(h) e^{\lambda_{2} t}+B(t, h),
$$


with $B(t, h):=\beta(t, h) e^{\left(\lambda_{2}-r^{\prime}\right) t}$. Here $\tilde{d}_{*}(z, h)=\int_{\mathbb{R}} e^{-z s} d_{*}(s, h) d s, \Re z \in\left(\left(\lambda_{2}\left(h_{0}\right)+\delta\right)(1+\right.$ $\gamma), 0)$, is the bilateral Laplace transform of $d_{*}(t, h)$. In view of the Lebesgue dominated convergence theorem and the uniform exponential estimate $d_{*}(t, h)=O\left(e^{\left(\lambda_{2}\left(h_{0}\right)+\delta\right)(1+\gamma) t}\right)$, $t \rightarrow+\infty, h \in \mathcal{O}^{\prime \prime}, \tilde{d}_{*}(z, h)$ depends continuously on $z, h$.

As consequence, there exists some small neighbourhood $\mathcal{O}^{\prime \prime \prime} \subset \mathcal{O}$ of $h_{0}$ such that continuous functions $\alpha(h), \beta(t, h)$ satisfy the estimates

$$
\alpha(h)=\frac{\tilde{d}_{*}\left(\lambda_{2}(h), h\right)}{\chi_{-}^{\prime}\left(\lambda_{2}(h)\right)}>0, \quad|\beta(t, h)| \leq \frac{1}{2 \pi} \int_{\mathbb{R}} \frac{\left|\tilde{d}_{*}\left(\lambda_{2}(h)-r^{\prime}+i s, h\right)\right|}{\left|\chi_{-}\left(\lambda_{2}(h)-r^{\prime}+i s\right)\right|} d s \leq B_{0}, \quad h \in \mathcal{O}^{\prime \prime \prime},
$$

where $B_{0}=B_{0}\left(\mathcal{O}^{\prime \prime \prime}\right)$ is some positive constant. Finally, integrating $(38)$ on $(t,+\infty)$, we find that

$$
\psi^{\prime}(t)=c \psi(t)+\int_{t}^{+\infty}\left(a_{-} \psi(s)+b_{-} \psi(s-h)-d_{*}(s, h)\right) d s=\lambda_{2}(h) \alpha(h) e^{\lambda_{2}(h) t}+R(t, h),
$$

where

$$
R(t, h)=c B(t, h)+\int_{t}^{+\infty}\left(a_{-} B(s, h)+b_{-} B(s-h, h)-d_{*}(s, h)\right) d s
$$

satisfies $|R(t, h)| \leq D e^{\left(\mu_{2}-r\right) t}, t>0, h \in \mathcal{O}^{\prime \prime \prime}$, with $D$ not depending on $h$.

Remark 5.10 Note that the representation (39) remains valid if the sub-tangency condition of Lemma 4.8 is replaced with the assumption $(\tau, c) \in \mathfrak{D}\left(\tilde{a}_{-}, \tilde{b}_{-}\right) \subset \mathfrak{D}\left(a_{-}, b_{-}\right)$. Indeed, in such a case, Lemma 4.3 assures that $q_{2}\left(h_{0}\right) \neq 0$.

\subsection{Global continuation of wavefronts.}

Now we can complete the proof of Theorems 1.3, 1.4. Consider the family $\mathfrak{F}$ of all continuous functions $c_{\alpha}:\left[0, h_{\alpha}\right) \rightarrow(0,+\infty), \alpha \in A$, such that for every $h=c \tau \in\left[0, h_{\alpha}\right)$ equation (2) has a bistable monotone wavefront propagating with the velocity $c_{\alpha}(h)$ and $c_{\alpha}(0)=c_{0}$ where $c_{0}$ is the speed of the unique bistable monotone front of the nondelayed equation. Lemmas 5.7, 5.9 show that $\mathfrak{F}$ is a non-empty set, $A \neq \emptyset$. We will introduce a partial order $\prec$ in $\mathfrak{F}$ in the following way: $\left(c_{\alpha}, h_{\alpha}\right) \prec\left(c_{\beta}, h_{\beta}\right)$ if $h_{\beta} \geq h_{\alpha}$ and $c_{\alpha}(h)=c_{\beta}(h)$ for all $h \in\left[0, h_{\alpha}\right)$. Clearly, we can apply the Zorn lemma to the family $(\mathfrak{F}, \prec)$, let $c^{*}:\left[0, h^{*}\right) \rightarrow(0,+\infty)$ be the maximal element. Note that if $\left(\mathbf{U}^{*}\right)$ is assumed then the graph $\mathfrak{G}$ of the curve $c^{*}$ belongs to the domain $\mathfrak{D}\left(a_{-}, b_{-}\right)$.

Suppose first that $h^{*}=+\infty$, then $\sup _{h>0} h / c^{*}(h)=+\infty$ since otherwise there exists a bounded sequence of delays $\tau_{j}=h_{j} / c^{*}\left(h_{j}\right), h_{j} \rightarrow+\infty$, such that $c^{*}\left(h_{j}\right)=h_{j} / \tau_{j} \rightarrow$ $+\infty$, contradicting to the conclusion of Lemma 5.3. In view of the intermediate value theorem, this implies that for each

$$
\tau \in[0,+\infty)=\left[\min _{h \geq 0} h / c^{*}(h), \sup _{h \geq 0} h / c^{*}(h)\right)
$$

there exists at least one monotone bistable wavefront.

Now, if $h^{*}<+\infty$, then, by Lemma $5.2, c^{*}$ is a bounded function on $\left[0, h^{*}\right)$. Let the interval $[p, q]$ (it can happen that $p=q$ ) denote the set of all partial limits of $c^{*}(h)$ 
as $h \rightarrow h^{*}$. Set $r=(p+q) / 2$ and suppose that $r>0$ (and, in addition, $r>c^{\mathfrak{E}}\left(h^{*}\right)$ if $\left(\mathbf{U}^{*}\right)$ is assumed). Then there exists a sequence $h_{j} \rightarrow h^{*}$ and $c_{j}=c^{*}\left(h_{j}\right)$ such that $c_{j} \rightarrow r$. The sequence of profiles $\phi_{j}$ of wavefronts $\phi_{j}\left(x+c_{j} t\right)$ is uniformly bounded and equicontinuous on $\mathbb{R}$. We can also assume that $\phi_{j}(0)=\left(e_{1}+e_{2}\right) / 2$ for every $j$. Therefore we can find a subsequence of $\phi_{j}$ (we will use the same notation $\phi_{j}$ for it) converging to some non-decreasing function $\phi_{*}$ such that $\phi_{*}(0)=\left(e_{1}+e_{2}\right) / 2$ and $\phi_{*}(t) \in\left[e_{1}, e_{3}\right], t \in \mathbb{R}$. It is easy to see that $\phi_{*}$ satisfies the differential equation

$$
\phi^{\prime \prime}(t)-r \phi^{\prime}(t)+g\left(\phi(t), \phi\left(t-h^{*}\right)\right)=0
$$

and $\phi_{*}( \pm \infty) \in\left\{e_{1}, e_{2}, e_{3}\right\}$. Actually, since $\phi_{*}$ is non-decreasing and $\phi_{*}(0)<e_{2}$, we obtain that $\phi_{*}(-\infty)=e_{1}$. Considering the possibility $\phi_{*}(+\infty)=e_{2}$, we find that $\phi_{*}(t) \in\left[e_{1}, e_{2}\right]$ for all $t \in \mathbb{R}$ and therefore $\phi_{*}^{\prime \prime}(t) \geq 0, t \in \mathbb{R}$. Clearly, this contradicts to the convergence of $\phi_{*}(t)$ at $+\infty$. Thus $\phi_{*}(t)$ is a strictly monotone bistable wavefront of the above limiting delay differential equation. Consequently, we can apply either Lemma 5.7 or Lemma 5.9 for parameters $c=r, h=h^{*}$ and conclude that there exists a smooth function $c=c(h)$ with $h$ from some open neighbourhood $\mathcal{U}$ of $h^{*}$ and a family of bistable wavefronts $\phi_{c(h)}, \phi_{r}=\phi_{*}$ for all $h \in \mathcal{U}$. In addition, if (U) is assumed, these wavefronts are monotone. Since $c(h)$ is smooth, we can use this function to extend continuously $c^{*}$ on the open interval $\left[0, h^{* *}\right)$ strictly bigger than $\left[0, h^{*}\right)$. This shows that either $r=0$ or $r=c^{\mathfrak{E}}\left(h^{*}\right)>0$.

As we have observed, under conditions of Theorem 1.3, the only case $r=0$ can happen. In such a case, the graph $\mathfrak{G}$ of $c^{*}$ connects continuously points $\left(0, c_{0}\right)$ and $\left(h^{*}, 0\right)$ so that for every fixed nonnegative $\tau$ the line $h=c \tau$ will intersect $\mathfrak{G}$ at least once at some point $(c(\tau) \tau, c(\tau))$. This means that if $r=0$ (in particular, this always occurs under conditions of Theorem 1.3) then for each fixed $\tau \geq 0$ the original equation has at least one bistable wavefront propagating with the velocity $c(\tau)>0$.

On the other hand, under conditions of Theorem 1.4, the situation when $c^{*}\left(h^{*}-\right)=$ $c^{\mathfrak{E}}\left(h^{*}\right)>0$ can also occur. Then the graph $\mathfrak{G}$ of $c^{*}$ connects continuously points $\left(0, c_{0}\right)$ and $\left(h^{*}, c^{*}\left(h^{*}\right)\right)$ so that for every fixed $\tau \in\left[0, \tau_{*}\right], \tau_{*}=h^{*} / c^{*}\left(h^{*}\right)$, the line $h=c \tau$ intersects $\mathfrak{G}$ at least once at some point $(c(\tau) \tau, c(\tau))$. Thus for each fixed $\tau \in\left[0, \tau_{*}\right]$ the original equation has at least one bistable wavefront propagating with the velocity $c(\tau)>0$. Next, due to the maximality property of $h^{*}$ there exist arbitrarily small positive $h-h^{*}$ such that $\left(h, c^{*}(h)\right) \notin \mathfrak{D}\left(a_{-}, b_{-}\right)$. Thus for delay $\tau^{\prime}=h / c^{*}(h)$ (which can be chosen arbitrarily close to $\left.\tau_{*}\right)$ there exists a non-monotone wavefront $\phi$ propagating with the velocity $c^{*}(h)$ close to $c^{\mathfrak{E}}\left(h^{*}\right)$. Moreover, since $\left(h, c^{*}(h)\right) \notin \mathfrak{D}\left(a_{-}, b_{-}\right)$, the leading asymptotic term of $e_{3}-\phi(t)$ at $+\infty$ is oscillatory, e.g. see [13, Lemma 4.6]. Thus $\phi(t)$ is oscillating around $e_{3}$ at $+\infty$.

Remark 5.11 If the sub-tangency condition of $\left(\mathbf{U}^{*}\right)$ is not assumed, the above proof remains true if we consider domain $\mathfrak{D}\left(\tilde{a}_{-}, \tilde{b}_{-}\right)$instead of $\mathfrak{D}\left(a_{-}, b_{-}\right)$. See Remarks 4.2, 4.10, 5.10. However, if $\mathfrak{D}\left(\tilde{a}_{-}, \tilde{b}_{-}\right) \neq \mathfrak{D}\left(a_{-}, b_{-}\right)$, then our approach does not allow to extend the curve $\mathfrak{G}$ till the boundary of $\mathfrak{D}\left(a_{-}, b_{-}\right)$making a conclusion about the existence 
of the oscillating wavefronts. It is worth noting that delayed reaction-diffusion equations can possess non-monotone non-oscillating wavefronts, cf. [10].

\section{Acknowledgements.}

The first author was supported by FONDECYT (Chile) under project 1150480. The second author was partially supported by the Ministry of Education and Science of the Russian Federation (the agreement number 02.a03.21.0008).

\section{References}

[1] Alfaro M, Ducrot A and Giletti T 2017 Travelling waves for a non-monotone bistable equation with delay: existence and oscillations Proc. London Math. Soc. doi:10.1112/plms.12092

[2] Ambrosetti A and Prodi G 1993 A primer of nonlinear analysis (Cambridge, Cambridge University Press)

[3] Apreutesei N, Ducrot A and Volpert V 2009 Travelling waves for integro-differential equations in population dynamics, Discrete Cont. Dyn. Syst. Ser. B 11 541-561

[4] Bani-Yaghoub M, Yao G-M, Fujiwara M and Amundsen D E 2015 Understanding the interplay between density dependent birth function and maturation time delay using a reaction-diffusion population model Ecological Complexity 21 14-26

[5] Berestycki H, Nadin G, Perthame B and Ryzhik L 2009 The non-local Fisher-KPP equation: travelling waves and steady states Nonlinearity 22 2813-2844

[6] Bocharov G, Meyerhans A, Bessonov N, Trofimchuk S and Volpert V 2016 Spatiotemporal dynamics of virus infection spreading in tissues PLoS ONE 11(12): e0168576. doi:10.1371/journal.pone. 0168576

[7] Bonnefon O, Garnier J, Hamel F and Roques L 2013 Inside dynamics of delayed traveling waves Math. Mod. Nat. Phen. 8 42-59

[8] Chow S-N, Lin X-B, Mallet-Paret J 1989 Transition layers for singularly perturbed delay differential equations with monotone nonlinearities J. Dynam. Differential Equations 1 3-43

[9] Ducrot A, Marion M, Volpert V 2011 Spectrum of some integro-differential operators and stability of travelling waves Nonlinear Analysis 74 4455-4473

[10] Ivanov A, Gomez C and Trofimchuk S 2014 On the existence of non-monotone non-oscillating wavefronts J. Math. Anal. Appl. 419 606-616

[11] Fang J and Zhao X-Q 2011 Monotone wavefronts of the nonlocal Fisher-KPP equation Nonlinearity 24 3043-3054

[12] Fang J and Zhao X-Q 2015 Bistable traveling waves for monotone semiflows with applications $J$. Eur. Math. Soc. 17 2243-2288

[13] Gomez A and Trofimchuk S 2014 Global continuation of monotone wavefronts J. London Math. Soc. $8947-68$

[14] Gourley S A, So J W-H and Wu J H 2004 Non-locality of reaction-diffusion equations induced by delay: biological modeling and nonlinear dynamics J. Math. Sci. 124 5119-5153

[15] Hale J K and Lin X-B 1985 Heteroclinic orbits for retarded functional differential equations $J$. Differential Equations 65 175-202

[16] Hale J K and Verduyn Lunel S M 1993 Introduction to functional differential equations, Applied Mathematical Sciences (Springer-Verlag)

[17] Hasik K, Kopfová J, Nábělková P and Trofimchuk S 2016 Traveling waves in the nonlocal KPPFisher equation: different roles of the right and the left interactions J. Differential Equations $2606130-6175$

[18] Huang W 2000 Monotonicity of heteroclinic orbits and spectral properties of variational equations for delay differential equations J. Differential Equations 162 91-139 
[19] Huang W 2001 Uniqueness of the bistable traveling wave for mutualist species J. Dynam. Differential Equations 13 147-183

[20] Hupkes H J and Verduyn Lunel S M 2005 Analysis of Newton's method to compute travelling waves in discrete media J. Dynam. Diff. Eqns. 17 523-572

[21] Hupkes H J and Verduyn Lunel S M 2003 Analysis of Newton's method to compute travelling wave solutions to lattice differential equations, Technical Report 2003-09, Mathematical Institute Leiden.

[22] Liang X and Zhao X-Q 2010 Spreading speeds and traveling waves for abstract monostable evolution systems J. Functional Anal. 259 857-903

[23] Martin R H and Smith H L 1990 Abstract functional differential equations and reaction-diffusion systems Trans. Amer. Math. Soc. 321 1-44

[24] Ma S and Wu J 2007 Existence, uniqueness and asymptotic stability of traveling wavefronts in a non-local delayed diffusion equation J. Dynam. Diff. Eqns. 19 391-436

[25] Mallet-Paret J 1999 The Fredholm alternative for functional differential equations of mixed type J. Dynam. Diff. Eqns. 11 1-48

[26] Nadin G, Rossi L, Ryzhik L and Perthame B 2013 Wave-like solutions for nonlocal reactiondiffusion equations: a toy model Math. Mod. Nat. Phen. 8 33-41

[27] Schaaf K 1987 Asymptotic behavior and travelling wave solutions for parabolic functional differential equations Trans. Amer. Math. Soc. 302 587-615

[28] Smith H L and Zhao X-Q 2000 Global asymptotic stability of traveling waves in delayed reactiondiffusion equations SIAM J. Math. Anal. 31 514- 534

[29] Trofimchuk S and Volpert V 2018 Travelling waves for a bistable reaction-diffusion equation with delay SIAM J. Math. Anal. $501175-1199$

[30] Volpert V 2011 Elliptic partial differential equations. Volume 1. Fredholm theory of elliptic problems in unbounded domains (Birkhäuser)

[31] Volpert A I, Volpert V A and Volpert V A 1994 Traveling Wave Solutions of Parabolic Systems, Translations of Mathematical Monographs, Vol. 140, (Amer. Math. Soc.:Providence)

[32] Wang Z-C, Li W-T and Ruan S 2007 Existence and stability of traveling wave fronts in reaction advection diffusion equations with nonlocal delay J. Differential Equations 238 153-200 